\title{
O Processo K em uma árvore de profundidade infinita
}

\author{
Gabriel Ribeiro da Cruz Peixoto
}

TESE APRESENTADA

$\mathrm{AO}$

Instituto DE MATEMÁticA E EstatísticA

DA

UNIVERSIDADE DE SÃO PAULO

PARA

OBTENÇÃO DO TÍTULO

$\mathrm{DE}$

DOUTOR EM CIÊNCIAS

Programa: Estatística

Orientador: Prof. Dr. Luiz Renato Gonçalves Fontes

Durante o desenvolvimento deste trabalho o autor recebeu auxílio financeiro da CAPES e CNPq

São Paulo, janeiro de 2015 


\title{
O Processo $\mathrm{K}$ em uma árvore de profundidade infinita
}

\begin{abstract}
Esta versão da dissertação/tese contém as correções e alterações sugeridas pela Comissão Julgadora durante a defesa da versão original do trabalho, realizada em 02/12/2014. Uma cópia da versão original está disponível no Instituto de Matemática e Estatística da Universidade de São Paulo.
\end{abstract}

Comissão Julgadora:

- Prof. Dr. Luiz Renato Gonçalves Fontes (orientador) - IME-USP

- Prof ${ }^{a}$. Dra ${ }^{a}$. Veronique Gayrard - AMU (França)

- Prof. Dr. Augusto Quadros Teixeira - IMPA

- Prof. Dr. Glauco Valle da Silva Coelho - UFRJ

- Prof. Dr. Christophe Frederic Gallesco - UNICAMP 


\section{Agradecimentos}

Gostaria de agradecer aos meus pais, que sempre me apoiaram em todas as empreitadas que eu me dispus a realizar e me deram amplas oportunidades para estudar e me aperfeiçoar.

Gostaria de agradecer meu orientador, Prof. Dr. Luiz Renato Fontes. Sua criatividade para encontrar novas abordagens para problemas, sua humildade e paciência sempre me causaram admiração e respeito. Tenho muito a agradecer pela liberdade que me foi propiciada durante esse trabalho para perseguir as abordagens que me pareciam mais promissoras.

Agradeço a todos os professores, colegas e funcionários do IME-USP. Com quem dividi espaço, assisti aulas e tive inúmeras conversas e discussões esclarecedoras, sejam sobre matemática ou não. Em particular agradeço ao NUMEC e todos ligados a ele por me acolher e hospedar.

Agradeço à minha família, por sempre estar lá e me apoiar. E aos meus amigos, com quem sempre pude contar para me divertir.

Por fim agradeço a banca examinadora pelas diversas críticas e sugestões feitas ao presente texto. 


\section{Resumo}

PEIXOTO, G. R. C O Processo K em uma árvore de profundidade infinita. 2015. 54 f. Tese (Doutorado) - Instituto de Matemática e Estatística, Universidade de São Paulo, São Paulo, 2015.

Nesse trabalho introduzimos o Processo K em árvores de profundidade infinita. Estes são processos estocásticos em tempo contínuo que têm $\overline{\mathbb{N}}_{*}^{\mathbb{N} *}$ como espaço de estados. O principal resultado desse trabalho consiste em mostrar que, sob condições adequadas, o processo título pode ser obtido como limite de Processos K em árvores de profundidade finita, estudados em Fontes et al. [FGG14], quando a profundidade delas cresce para o infinito.

Palavras-chave: Processos K, Limites de processos, GREM. 


\section{Abstract}

PEIXOTO, G. R. C. The K-Process on a tree with infinite depth. 2015. $54 \mathrm{f}$. Tese (Doutorado) - Instituto de Matemática e Estatística, Universidade de São Paulo, São Paulo, 2015.

We introduce the K-Process on trees with infinite depth. These are stochastic processes in continuous time, having state space $\overline{\mathbb{N}}_{*}^{\mathbb{N} *}$. As the main result from this thesis, we prove that, under adequate assumptions, the title process can be obtained as the limit of K-Process on trees with finite depth, studied in Fontes et al. [FGG14], as the depth of the tree grows to infinity.

Keywords: K-Processes, Limits of Processes, GREM. 


\section{Sumário}

1 Introdução $\quad 1$

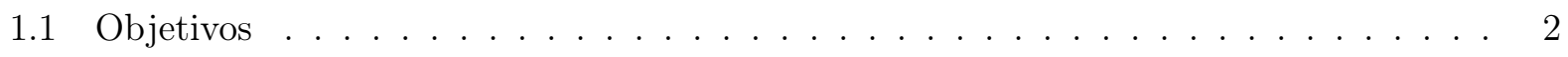

1.2 Organização do Trabalho . . . . . . . . . . . . . . . . . . . 2

2 O Processo K em uma árvore de profundidade finita 3

2.1 Construção do Processo K em árvores de profundidade finita . . . . . . . . . . . . . 3

3 Relógios Limite $\quad 13$

3.1 Limites do meio aleatório . . . . . . . . . . . . . . . . . 13

3.2 Relógios modificados . . . . . . . . . . . . . . . . . . . . 16

3.3 Trivialidade dos Relógios Limite . . . . . . . . . . . . . . . . . . . . . . . . 21

4 O Processo K em uma Árvore de Profundidade Infinita 29

4.1 Definição do Processo . . . . . . . . . . . . . . . . . . . . . . . . . . . . 29

4.2 Convergência dos Relógios . . . . . . . . . . . . . . . . . . . . 29

4.3 Propriedades do Processo K em uma árvore de profundidade infinita . . . . . . . . . 35

4.4 Convergência do Processo K . . . . . . . . . . . . . . . . . . . 38

4.5 Medida Empírica . . . . . . . . . . . . . . . . . . . . . . . . . 42

$\begin{array}{lll}5 & \text { Conclusões } & 49\end{array}$

$\begin{array}{ll}\text { A Resultados auxiliares } & 51\end{array}$ 


\section{Capítulo 1}

\section{Introdução}

Modelos de armadilhas foram introduzidos como modelos caricatura, usados para tentar entender o comportamento a longo prazo de dinâmicas de vidros de spin em baixa temperatura, particularmente o Random Energy Model (REM) e o Generalized Random Energy Model (GREM) [Bou92; BD95].

Esses modelos de armadilha normalmente são passeios aleatórios simples em tempo contínuo, com a particularidade de que o tempo médio que o processo passa em cada estado também é aleatório, escolhido a partir de uma distribuição com cauda pesada. Escolhas usuais para essas distribuições, advindas dos vidros de spin, são leis na bacia de atração de uma distribuição $\alpha$ estável, com $\alpha \in(0,1)$. Estas são distribuições com primeiro momento infinito.

Processos K são encontrados como limites de escala desses modelos de armadilhas, numa escala de tempo que permita a visita às armadilhas mais profundas. Eles foram introduzidos por Fontes e Mathieu [FM08], como um limite de escala do modelo de armadilhas em um grafo completo, modelo este que é relacionado ao REM. Os autores também o relacionaram ao exemplo K1 de Kolmogorov [Kol51], de onde o nome Processo K se originou.

Fontes e Lima [FL09] mostraram a convergência da Random Hopping Times dynamics do REM no hipercubo para o Processo K.

Bezerra et al. $[\mathrm{Bez}+12]$ introduziram e estudaram o Processo $\mathrm{K}$ com pesos como um limite de escalas de modelos de armadilha assimétricos em grafos completos. Fontes e Peixoto [FP13] estudaram esse modelo mais a fundo, descrevendo seu gerador, entre outros resultados [veja também Pei11].

Jara et al. [JLT12] provaram que Processos K com pesos podem ser obtidos como limites de escala em uma classe grande de grafos, incluindo o hipercubo, o Toro, grafos d-regulares aleatórios e a maior componente conexa do grafo de Erdös-Renyi.

Um tipo particular de modelo de armadilha, relacionado ao GREM e introduzido por Sasaki e Nemoto [SN00] foi considerado em Fontes et al. [FGG14]. Neste artigo os autores introduziram um novo tipo de Processo K, que chamaremos de Processo K numa árvore de profundidade finita, e mostraram que ele pode ser obtido como limite de escala desses modelos de armadilha. [veja também Gav11] 


\subsection{Objetivos}

Nesta tese iremos introduzir o Processo K numa árvore com profundidade infinita e provar que, sob condições adequadas, ele é o limite de Processos K em árvores de profundidade finita, quando a profundidade delas cresce para o infinito.

\subsection{Organização do Trabalho}

O Capítulo 2 descreve uma construção do Processo K em profundidade finita, que é uma adaptação da construção empregada em [FGG14]. Também provamos algumas propriedades básicas desse processo que serão úteis para o desenvolver dos nossos resultados.

O Capítulo 3 constrói os relógios limite, processos estocásticos auxiliares que serão fundamentais na construção do Processo $\mathrm{K}$ em uma árvore de profundidade infinita. Também provaremos os Teoremas 3.7 e 3.8, em que daremos condições sobre as quais esses relógios limite são ou não triviais.

Finalmente no Capítulo 4 iremos definir o processo título, provando algumas de suas propriedades básicas e culminando no Teorema 4.15, que dá condições para que os Processos K em árvores de profundidade finita convirjam para o processo em árvores de profundidade infinita. Por fim, na Seção 4.5, iremos calcular uma medida empírica do processo título, isso é, a proporção do tempo onde que nosso processo permanece em cada possível estado. Isso dá uma sugestão para qual deve ser uma medida de equilíbrio do Processo K em uma árvore de profundidade infinita.

O Apêndice A traz alguns resultados auxiliares, que não se referem diretamente ao problema em questão e que por essa razão optamos por não incluir no corpo do texto. 


\section{Capítulo 2}

\section{O Processo K em uma árvore de profundidade finita}

Nesse capítulo vamos apresentar o Processo K em uma árvore de profundidade finita, introduzido por Fontes et al. [FGG14]. Muitos dos elementos dessa construção serão usados quando definirmos o Processo K em árvores de profundidade infinita.

Todos os resultados deste capítulo foram retirados de Fontes et al. [FGG14] e Gava [Gav11] e adaptados quando necessário. O objetivo desse capítulo é contextualizar o leitor, bem como estabelecer notações e definições sobre as quais vamos basear nossos resultados.

\subsection{Construção do Processo $\mathrm{K}$ em árvores de profundidade finita}

O espaço de estados de um Processo $\mathrm{K}$ em uma árvore de profundidade $k$ é $\overline{\mathbb{N}}_{*}^{k}$, onde $\mathbb{N}_{*}=$ $\{1,2, \ldots\}$ e $\overline{\mathbb{N}}_{*}=\mathbb{N}_{*} \cup\{\infty\}$. Aqui trataremos $\infty$ como um símbolo novo a ser adicionado aos números naturais. Esse símbolo terá uma interpretação natural como infinito quando munirmos esse espaço de uma topologia, que será uma compactificação dos números naturais.

Vamos denotar elementos desse espaço por $\left.x\right|_{k}=\left(x_{1}, \ldots, x_{k}\right)$. Para simplificar a notação, iremos frequentemente escrever $\left.x\right|_{k}=x_{1} x_{2} \ldots x_{k}$. Iremos ainda usar a notação $\left.x\right|_{k} y$ para representar a concatenação de $\left.x\right|_{k} \operatorname{com} y$, isso é, $\left.x\right|_{k} y=\left(x_{1}, \ldots, x_{k}, y\right) \in \mathbb{N}_{*}^{k+1}$.

Será útil considerar os elementos de $\overline{\mathbb{N}}_{*}^{k}$ como as folhas de uma árvore de profundidade $k$. Tal árvore tem $\varnothing$ como raiz e um nó $x_{j} y$ é filho de $x_{j}$. Essa é a árvore no título do processo, representada na Figura 2.1.

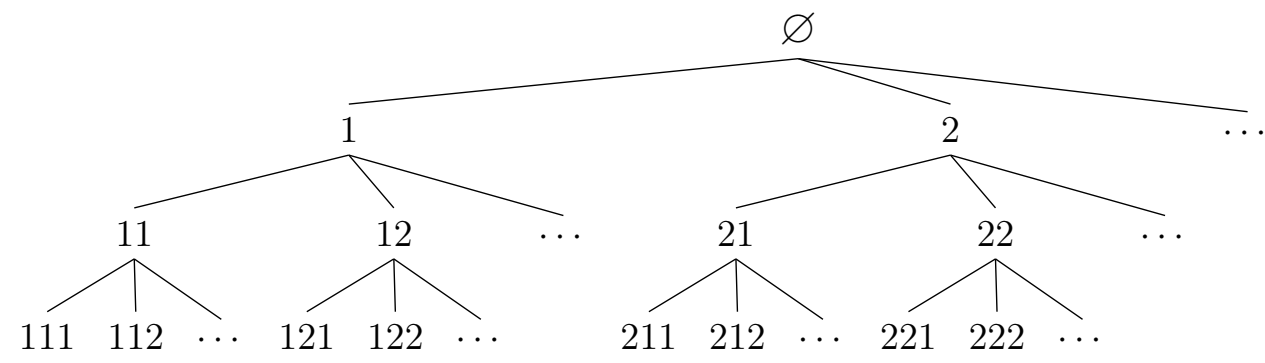

Figura 2.1: Representação do espaço de estados por uma árvore

Os parâmetros do processo serão uma sequência $0<\alpha_{1}<\ldots<\alpha_{k}<\ldots<1$ de números reais, 
tais que $\alpha_{k} \rightarrow 1$ quando $k \rightarrow \infty$.

Construiremos o processo num espaço de probabilidade que admita as seguintes famílias de variáveis aleatórias independentes:

- $\left\{\gamma_{k}\left(\left.x\right|_{k}\right):\left.x\right|_{k} \in \mathbb{N}_{*}^{k}, k \in \mathbb{N}_{*}\right\}:$ onde $\gamma_{k}\left(\left.x\right|_{k-1} 1\right)>\gamma_{k}\left(\left.x\right|_{k-1} 2\right)>\ldots>0$ são a estatística de ordem das marcas de um Processo Pontual de Poisson em $\mathbb{R}^{+}$com medida intensidade $\mu_{k}$ :

$$
\mu_{k}(d t):=\frac{c_{k}}{t^{1+\alpha_{k}}}, t>0, \quad \text { onde } \quad c_{k}:=\frac{\alpha_{k}}{\Gamma\left(1-\frac{\alpha_{k}}{\alpha_{k+1}}\right)},
$$

$\Gamma$ é a função Gama tradicional.

- $\left\{T_{i}^{k, x}: i, k, x \in \mathbb{N}_{*}\right\}:$ uma família de variáveis aleatórias independentes e identicamente distribuídas (i.i.d.), cada uma com distribuição exponencial de taxa 1;

- $\left\{N^{k, x}: k, x \in \mathbb{N}_{*}\right\}:$ uma família de Processos de Poisson independentes, cada um com taxa 1 . As marcas de $N^{k, x}$ serão indicadas por $0<\sigma_{1}^{k, x}<\sigma_{2}^{k, x}<\ldots$.

Definição 2.1. Vamos nos referir à família $\gamma:=\left\{\gamma_{k}\left(\left.x\right|_{k}\right):\left.x\right|_{k} \in \mathbb{N}_{*}^{k}, k \in \mathbb{N}\right\}$ como o meio aleatório onde o Processo K está inserido. Frequentemente iremos calcular esperanças que não integram sobre esse meio aleatório, iremos denota-las por $\mathbb{E}_{\gamma}$.

Observação 2.1. A construção que vamos apresentar para o Processo $K$ em árvores de profundidade finita faz sentido mesmo quando fazemos uma escolha diferente para $\left\{\gamma_{k}\left(\left.x\right|_{k}\right)\right\}$, seja essa escolha aleatória ou determinística. Só precisaremos que essa família satisfaça quase certamente:

$$
\sum_{\left.x\right|_{k} \in \mathbb{N}_{*}^{k}} \bar{\gamma}_{k}\left(\left.x\right|_{k}\right)<\infty, \quad \text { onde } \quad \bar{\gamma}_{k}\left(\left.x\right|_{k}\right):=\prod_{j=1}^{k} \gamma_{j}\left(\left.x\right|_{j}\right) .
$$

Vamos provar essa propriedade para a nossa escolha na Proposição 2.2 abaixo.

A lei que nós escolhemos para o meio aleatório, a menos da escolha das constantes $c_{k}$, foi tratada em outros textos pois ela é o que se obtêm ao tomar limites de escalas de modelos de armadilha baseados no GREM [FGG14]. Qualquer escolha para $c_{k}$ é possivel nesse caso. Nós adotamos esse valor especifico para $c_{k}$ para garantir a convergência da Proposição 3.1.

Definição 2.2. Para $k, n \in \mathbb{N}_{*}$ fixados, $k \leqslant n$, e $\left.x\right|_{k} \in \overline{\mathbb{N}}_{*}^{k}$ vamos denotar por cilindros baseados em $\left.x\right|_{k}$ como:

$$
\begin{aligned}
& {\left[\left.x\right|_{k}\right]_{n}:=\left\{\left.y\right|_{n} \in \mathbb{N}_{*}^{n}:\left.y\right|_{k}=\left.x\right|_{k}\right\},} \\
& \overline{\left[\left.x\right|_{k}\right]_{n}}:=\left\{\left.y\right|_{n} \in \overline{\mathbb{N}}_{*}^{n}:\left.y\right|_{k}=\left.x\right|_{k}\right\} .
\end{aligned}
$$

Proposição 2.2. Nossa escolha para $\left\{\gamma_{k}\left(\left.x\right|_{k}\right): k \in \mathbb{N}_{*},\left.x\right|_{k} \in \mathbb{N}_{*}^{k}\right\}$ satisfaz (2.2) quase certamente. Além disso, para todo $0 \leqslant j<k$ e $\left.x\right|_{j}$ fixados, podemos calcular a seguinte transformada de Laplace:

$$
\mathbb{E}\left[\exp \left\{-\lambda \sum_{\left.y\right|_{k} \in\left[\left.x\right|_{j}\right]_{k}} \frac{\bar{\gamma}_{k}\left(\left.y\right|_{k}\right)}{\bar{\gamma}_{j}\left(\left.x\right|_{j}\right)}\right\}\right]=\exp \left\{-\left[\frac{\Gamma\left(1-\alpha_{k}\right)}{\Gamma\left(1-\frac{\alpha_{k}}{\alpha_{k+1}}\right)}\right]^{\alpha_{j+1} / \alpha_{k}} \lambda^{\alpha_{j+1}}\right\}
$$


Demonstração. Podemos identificar (2.3) como a transformada de Laplace de uma distribuição estável com índice de estabilidade $\alpha_{j+1}$. Essa variável é portanto finita quase certamente.

Vamos provar (2.3) por indução em $j$. A base é o caso $j=k-1$. Usando o Teorema de Campbell, encontrado, por exemplo, na Seção 3.2 de Kingman [Kin93], podemos calcular:

$$
\begin{aligned}
\mathbb{E}\left[\exp \left\{-\lambda \sum_{x_{k} \in \mathbb{N}_{*}} \gamma_{k}\left(\left.x\right|_{k}\right)\right\}\right] & =\exp \left\{-\int_{0}^{\infty}\left(1-e^{-\lambda t}\right) \mu_{k}(d t)\right\} \\
& =\exp \left\{\lambda^{\alpha_{k}} \frac{\Gamma\left(1-\alpha_{k}\right)}{\Gamma\left(1-\alpha_{k} / \alpha_{k+1}\right)}\right\}
\end{aligned}
$$

Isso conclui o caso $j=k-1$. Para o passo da indução, podemos usar novamente o Teorema de Campbell, junto com as Proposições A.1 e A.3, para calcular:

$$
\begin{aligned}
& \mathbb{E}\left[\exp \left\{-\lambda \sum_{\left.y\right|_{k} \in\left[\left.x\right|_{j}\right]_{k}} \frac{\bar{\gamma}_{k}\left(\left.y\right|_{k}\right)}{\bar{\gamma}_{j}\left(\left.y\right|_{j}\right)}\right\}\right]=\mathbb{E}\left[\exp \left\{-\lambda \sum_{x_{j+1}} \gamma_{j+1}\left(\left.x\right|_{j+1}\right) \sum_{\left.y\right|_{k} \in\left[\left.x\right|_{j+1}\right]_{k}} \frac{\bar{\gamma}_{k}\left(\left.y\right|_{k}\right)}{\bar{\gamma}_{j}\left(\left.y\right|_{j+1}\right)}\right\}\right] \\
& =\exp \left\{-\int_{0}^{\infty}\left(1-e^{-\lambda t}\right) \mathbb{E}\left[\left(\sum_{\left.y\right|_{k} \in\left[\left.x\right|_{j+1}\right]_{k}} \frac{\bar{\gamma}_{k}\left(\left.y\right|_{k}\right)}{\overline{\bar{\gamma}}_{j}\left(\left.y\right|_{j+1}\right)}\right)^{\alpha_{j+1}}\right] \mu_{j+1}(d t)\right\} \\
& =\exp \left\{-\left[\frac{\Gamma\left(1-\alpha_{k}\right)}{\Gamma\left(1-\frac{\alpha_{k}}{\alpha_{k+1}}\right)}\right]^{\alpha_{j+1} / \alpha_{k}} \lambda^{\alpha_{j+1}}\right\} .
\end{aligned}
$$

Iremos construir o Processo K e um processo auxiliar, chamado Processo Relógio, simultaneamente de maneira recursiva. Suponha $X_{0} \equiv \varnothing$ e defina para $t \geqslant 0$ e $k=1,2, \ldots$ :

$$
\begin{gathered}
\Xi_{k}(t):=\sum_{x \in \mathbb{N}_{*}} \sum_{i=1}^{N^{k, x}(t)} \gamma_{k}\left(X_{k-1}\left(\sigma_{i}^{k, x}\right) x\right) T_{i}^{k, x} \\
X_{k}(t):= \begin{cases}\left(X_{k-1}\left(\Xi_{k}^{-1}(t)\right), x\right) & \text { if } t \in \bigcup_{i=1}^{\infty}\left[\Xi_{k}\left(\sigma_{i}^{k, x}-\right), \Xi_{k}\left(\sigma_{i}^{k, x}\right)\right) \\
\left(X_{k-1}\left(\Xi_{k}{ }^{-1}(t)\right), \infty\right) & \text { caso contrário, }\end{cases}
\end{gathered}
$$

onde $\Xi_{k}^{-1}(t)=\inf \left\{r \geqslant 0: \Xi_{j}(r)>t\right\}$ é a inversa generalizada de $\Xi_{k}$.

Definição 2.3. $X_{k}$ é o Processo $K$ em uma árvore de profundidade $k$. Os processos $\Xi_{k}$ serão chamados de Processos Relógio.

Observação 2.3. Os Processos relógios são usualmente denotados na literatura pela letra grega gama (Г). Nós optamos por mudar essa convenção para evitar que estes sejam confundidos com a função Gama, $\Gamma(\alpha)=\int_{0}^{\infty} e^{-x} x^{\alpha-1} d x$.

A Figura 2.2 exemplifica essa construção em uma árvore de profundidade 2 para o caso onde $\gamma_{1}(x)=0$ e $\gamma_{2}(x y)=0$ sempre que $x \notin\{1,2\}$ ou $y \notin\{1,2\}$. Os intervalos representados no eixo y representam "intervalos de constância" para o Processo K naquele nível, enquanto que os intervalos no eixo x representam os intervalos de constância do nível anterior.

Note que, como impomos que $\gamma_{j}\left(\left.x\right|_{j}\right)>0$ para todo $\left.x\right|_{j} \in \mathbb{N}_{*}^{j}$, então essa figura não representa uma situação dentro das nossas suposições. Dentro das nossas condições, os relógios $\Xi_{j}$ são estrita- 


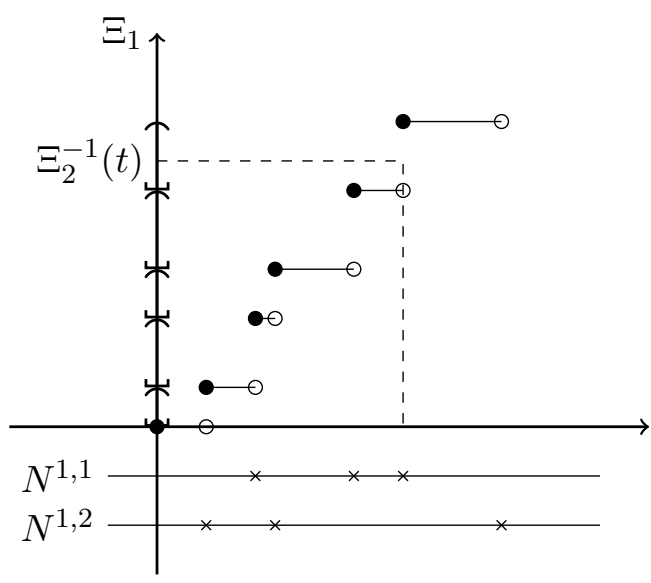

(a) Primeiro nivel

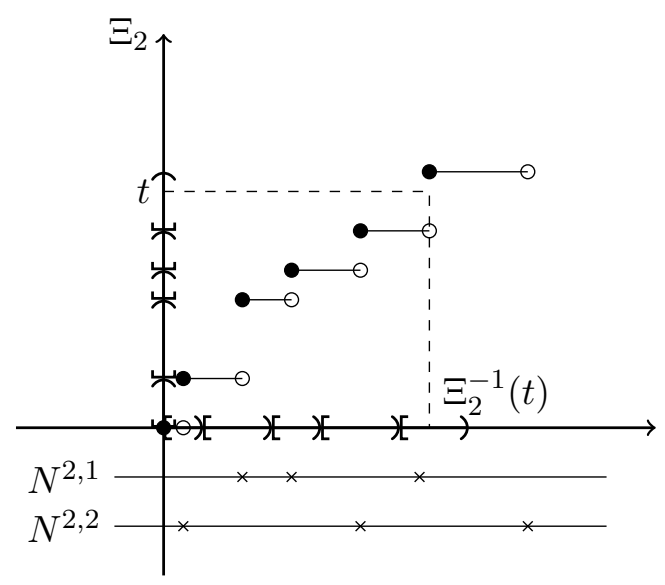

(b) Segundo nivel

Figura 2.2: Construção simplificada de um Processo $K$ com dois níveis.

mente crescentes com probabilidade 1 (Proposição 2.4). Porém ela é uma boa ilustração para fins didáticos.

As linhas tracejadas indicam como podemos encontrar o valor de $X_{2}(t)$ para um $t$ em particular. Nesse caso $X_{2}^{F}(t)=(1,1)$, já que o salto correspondente à esse valor de $t$ veio do primeiro Processo de Poisson no segundo nível $\left(N^{2,1}\right)$ e também veio do primeiro processo no primeiro nível $\left(N^{1,1}\right)$.

Definição 2.4. Para $k \leqslant n$, definimos o relógio composto como:

$$
\theta_{k}^{n}:=\Xi_{n} \circ \Xi_{n-1} \circ \cdots \circ \Xi_{k}
$$

Por conveniência, também iremos nos referir a esses processos como relógios.

Proposição 2.4. Para cada $k \in \mathbb{N}_{*}, t \geqslant 0, \Xi_{k}(t)$ é quase certamente finito. Além disso, $\Xi_{k}$ é quase certamente uma função escada, contínua a direita e estritamente crescente e $\lim _{t \rightarrow \infty} \Xi_{k}(t)=\infty$.

Demonstração. Vamos provar apenas que $\Xi_{k}(t)$ é finito q.c. , as demais afirmações dessa proposição podem ser obtidas diretamente da construção.

Para isso vamos mostrar que, para quase toda realização fixada do meio aleatório $\gamma=\left\{\gamma_{k}\left(\left.x\right|_{k}\right)\right.$ : $\left.k \in \mathbb{N}_{*},\left.x\right|_{k} \in \mathbb{N}_{*}^{k}\right\}$, vale que:

$$
\mathbb{E}_{\gamma}\left(\theta_{1}^{k}(t)\right)=t \sum_{\left.x\right|_{k} \in \mathbb{N}_{*}^{k}} \bar{\gamma}_{k}\left(\left.x\right|_{k}\right),
$$

lembrando, da Definição 2.1, que $\mathbb{E}_{\gamma}$ é uma esperança onde não integramos em $\gamma$.

Provaremos isso por indução em $k$. O caso $k=1$ pode ser verificado diretamente utilizando o Teorema de Fubini.

Para $k>1$, observe que $\theta_{2}^{k}$, dentro de um intervalo $\left[\Xi_{1}\left(\sigma_{i}^{k, x_{1}}-\right), \Xi_{1}\left(\sigma_{i}^{k, x_{1}}\right)\right)$, se comporta exatamente como $\theta_{1}^{k-1}$ para o valor fixado de $x_{1}$. Dessa forma, se $\mathcal{F}_{1}$ é a $\sigma$-álgebra gerada pelas variáveis usadas na construção no primeiro nível, usando a hipótese de indução, a independência das variáveis usadas na construção e o fato de Processos de Poisson terem incrementos independentes e 
estacionários, podemos escrever:

$$
\begin{aligned}
\mathbb{E}_{\gamma}\left(\theta_{1}^{k}(t)\right) & =\mathbb{E}_{\gamma}\left[\mathbb{E}_{\gamma}\left(\theta_{2}^{k}\left(\Xi_{1}(t)\right) \mid \mathcal{F}_{1}\right)\right] \\
& =\mathbb{E}_{\gamma}\left[\sum_{x_{1}} \Xi_{1}(t) \sum_{\left.y\right|_{k} \in\left[\left.x\right|_{1}\right]_{k}} \frac{\bar{\gamma}_{k}\left(\left.y\right|_{k}\right)}{\bar{\gamma}_{1}\left(\left.x\right|_{1}\right)}\right] \\
& =t \sum_{\left.x\right|_{k}} \bar{\gamma}_{k}\left(\left.x\right|_{k}\right) .
\end{aligned}
$$

Proposição 2.5. A medida de Lebesgue da imagem de $\Xi_{k}$ vale zero quase certamente para todo $k$.

Demonstração. $\Xi_{k}$ é uma função escada, isso é, existe um conjunto $S \subseteq \mathbb{R}^{+}$enumerável e uma função $\pi: S \rightarrow(0, \infty)$ tal que para todo $t \geqslant 0$ :

$$
\Xi_{k}(t)=\sum_{s \in S, s \leqslant t} \pi(s)
$$

Seja $\mathcal{R}(t):=\left\{\Xi_{k}(s): s \leqslant t\right\}$. Como $\Xi_{k}$ é estritamente crescente, isso é, $S$ é denso em $(0, \infty)$, então $\left(\Xi_{k}(s-), \Xi_{k}(s)\right) \cap \mathcal{R}(t)=\varnothing$ para todo $t>0$ e $s \in S$. O intervalo $(\Xi(s-), \Xi(s))$ tem comprimento $\pi(s)$.

Logo, sendo $l$ a medida de Lebesgue, temos que:

$$
l(\mathcal{R}(t))=\Xi_{k}(t)-\sum_{s \in S, s \leqslant t} \pi(s)=0
$$

Se tomarmos $\left(t_{n}\right)$ uma sequência crescente de números reais tais que $t_{n} \rightarrow \infty$ quanto $n \rightarrow \infty$, então a imagem de $\Xi_{k}$ é dada pela união de $l\left(\mathcal{R}\left(t_{n}\right)\right)$. Como essa é uma união enumerável de conjuntos que têm medida de Lebesgue zero, concluímos que a imagem de $\Xi_{k}$ também tem medida de Lebesgue zero.

Lema 2.6. Seja $f: \mathbb{R} \rightarrow \mathbb{R}$ uma função monótona não decrescente, tome $f^{-1}(t):=\inf \{r \geqslant 0$ : $f(r)>t\}$ sua inversa generalizada, $0<a<b<\infty$ et $t \geqslant 0$ números reais tais que $f$ seja contínua em a e b. Nessas condições:

$$
f^{-1}(t) \in[a, b) \Leftrightarrow t \in[f(a), f(b)) .
$$

Demonstração. Defina $A_{t}=\{r \geqslant 0: f(r)>t\}$.

Primeiro suponha que $t<f(b)$. Como $f$ é contínua em $b$ então existe um $\epsilon>0$ tal que $f(b-\epsilon)>t$. Portanto $b-\epsilon \in A_{t} \Rightarrow b-\epsilon \geqslant \inf A_{t}=f^{-1}(t)$. Portanto $b>f^{-1}(t)$.

Agora suponha que $f^{-1}(t)<b \Rightarrow \inf A_{t}<b$. Como $f$ é não decrescente, isso implica que $b \in A_{t} \Rightarrow f(b)>t$.

Com isso mostramos que, para todo $b$ ponto de continuidade de $f, t<f(b)$ se e somente se $f^{-1}(t)<b$.

Como $a$ também é um ponto de continuidade de $f$, podemos tomar a negação das duas afirmações, substituindo $b$ por $a$ e obteremos que $t \geqslant f(a)$ se e somente se $f^{-1}(t) \geqslant a$, o que completa a prova. 
Proposição 2.7. Para todo $t \geqslant 0, i, j, k, x \in \mathbb{N}_{*}, j \leqslant k$, vale que $\theta_{j}^{k}(t), \theta_{j}^{k}\left(\sigma_{i}^{j, x}-\right)$ e $\theta_{j}^{k}\left(\sigma_{i}^{j, x}\right)$ têm distribuições absolutamente contínuas em relação à medida de Lebesgue.

Demonstração. Primeiramente vamos mostrar que $\theta_{j}^{k}(t)$ tem distribuição absolutamente contínua com relação à medida de Lebesgue.

Podemos escrever $\theta_{j}^{k}(t)$ como:

$$
\theta_{j}^{k}(t)=\sum_{i} \tilde{\gamma}_{i} \tilde{T}_{i}
$$

onde as famílias $\left\{\tilde{\gamma}_{i}\right\}$ e $\left\{\tilde{T}_{i}\right\}$ são independentes entre si e $\left\{T_{i}\right\}$ são variáveis i.i.d., exponenciais de média 1. Fazemos isso enumerando as marcas $\left\{\sigma_{i}^{k, x}: \sigma_{i}^{k, x} \leqslant \theta_{j}^{k-1}(t)\right\}$ referentes à $\Xi_{k}$ e tomando os valores de $\gamma_{k}\left(\left.x\right|_{k}\right)$ e $T_{i}^{k, x}$ correspondentes.

Dessa forma, como cada $\tilde{T}_{i}$ têm uma distribuição contínua com relação à medida de Lebesgue, concluímos que $\theta_{j}^{k}(t)$ também têm.

Agora vamos estudar $\theta_{j}^{k}\left(\sigma_{i}^{j, x}-\right)$ e $\theta_{j}^{k}\left(\sigma_{i}^{j, x}\right)$. Para tratar o caso $j=k$, perceba que:

$$
\begin{aligned}
\Xi_{j}\left(\sigma_{i}^{j, x}-\right) & =\sum_{y \neq x} \sum_{l=1}^{N^{j, y}\left(\sigma_{i}^{j, x}\right)} \gamma_{j}\left(X_{j-1}\left(\sigma_{l}^{j, y}\right) x\right) T_{l}^{j, y}+\sum_{l<i} \gamma_{j}\left(X_{j-1}\left(\sigma_{l}^{j, x}\right) x\right) T_{l}^{j, x}, \\
\Xi_{j}\left(\sigma_{i}^{j, x}\right) & =\sum_{y \neq x} \sum_{l=1}^{N^{j, y}\left(\sigma_{i}^{j, x}\right)} \gamma_{j}\left(X_{j-1}\left(\sigma_{l}^{j, y}\right) x\right) T_{l}^{j, y}+\sum_{l \leqslant i} \gamma_{j}\left(X_{j-1}\left(\sigma_{l}^{j, x}\right) x\right) T_{l}^{j, x} .
\end{aligned}
$$

Dessa forma, por argumento análogo ao usado acima, $\Xi_{j}\left(\sigma_{i}^{j, x}-\right)$ e $\Xi_{j}\left(\sigma_{i}^{j, x}\right)$ têm distribuições absolutamente contínuas em relação à medida de Lebesgue.

O caso $k>j$ pode ser obtido ao perceber que:

$$
\begin{aligned}
\theta_{j}^{k}\left(\sigma_{i}^{j, x}-\right) & =\theta_{j+1}^{k}\left(\Xi_{j}\left(\sigma_{i}^{j, x}-\right)\right), \\
\theta_{j}^{k}\left(\sigma_{i}^{j, x}\right) & =\theta_{j+1}^{k}\left(\Xi_{j}\left(\sigma_{i}^{j, x}\right)\right) .
\end{aligned}
$$

Portanto tanto $\theta_{j}^{k}\left(\sigma_{i}^{j, x}-\right)$ quanto $\theta_{j}^{k}\left(\sigma_{i}^{j, x}\right)$ podem ser obtidos como misturas da família $\left\{\theta_{j+1}^{k}(t)\right.$ : $t \geqslant 0\}$ e dessa forma têm distribuições absolutamente contínuas em relação à medida de Lebesgue.

Proposição 2.8. Se $X_{k, j}$ é a j-ésima coordenada de $X_{k}, j \leqslant k$, então

$$
X_{k, j}(t)= \begin{cases}x & \text { se } t \in \bigcup_{i=1}^{\infty}\left[\theta_{j}^{k}\left(\sigma_{i}^{j, x}-\right), \theta_{j}^{k}\left(\sigma_{i}^{j, x}\right)\right) \\ \infty & \text { caso contrário. }\end{cases}
$$

Demonstração. Se $j=k$, essa propriedade é obtida diretamente da construção. Dessa maneira o caso $k=1$ está coberto. Vamos supor daqui para frente que $j<k$.

Como hipótese de indução, suponha que (2.7) vale para $k-1$ e mostra-la para $k$. Observando a construção do processo em (2.4b), notamos que, para $j<k$ :

$$
X_{k, j}(t)=X_{k-1, j}\left(\Xi_{k}^{-1}(t)\right)
$$

Portanto a hipótese de indução nos diz que $X_{k, j}(t)=x$ se e somente se $\Xi_{k}^{-1}(t) \in\left[\theta_{j}^{k-1}\left(\sigma_{i}^{j, x}-\right), \theta_{j}^{k-1}\left(\sigma_{i}^{j, x}\right)\right)$ 
para algum $i \in \mathbb{N}_{*}$.

A Proposição 2.7 nos diz que os dois extremos desse intervalo têm distribuição absolutamente contínua em relação à medida de Lebesgue. Dessa forma, quase certamente, não há um "empate" entre esses pontos e o conjunto $\left\{\sigma_{i}^{k, y}: i, y \in \mathbb{N}_{*}\right\}$, que são os pontos de descontinuidade de $\Xi_{k}$.

Portanto podemos aplicar o Lema 2.6 para concluir (2.7).

Proposição 2.9. Para todo $j \leqslant k$, o conjunto dos pontos de descontinuidade de $\theta_{j}^{k}$ é dado q.c. por $\left\{\sigma_{i}^{j, x}: i, x \in \mathbb{N}_{*}\right\}$.

Demonstração. Se $j=k$, então esse é um resultado direto da construção. Assim vamos supor que $j<k$.

Por construção, o conjunto dos pontos de descontinuidade de $\theta_{j}^{k}$ é dado por:

$$
\left\{\sigma_{i}^{j, x}: i, x \in \mathbb{N}_{*}\right\} \cup\left\{s \geqslant 0: \theta_{j}^{l}(s)=\sigma_{i}^{l+1, x} \text { para algum } i, x \in \mathbb{N}_{*}, j \leqslant l<k\right\} .
$$

Porém o segundo conjunto dessa expressão é vazio q.c. . Isso porque a Proposição 2.7 noz diz que a medida de Lebesgue da imagem de $\theta_{j}^{l}$ vale zero q.c. . Dessa forma cada $\sigma_{i}^{l+1, x}$ tem probabilidade zero de pertencer à essa imagem. Como temos uma quantidade enumerável de $\sigma_{i}^{l+1, x}$, então segue que a interseção desses conjuntos é vazia q.c. .

Proposição 2.10. Para todo $j \leqslant k<n$ :

$$
X_{k, j}(t)=X_{n, j} \circ \theta_{k+1}^{n}(t) .
$$

Demonstração. Fixemos um $x \in \mathbb{N}_{*}$ e vamos mostrar que $X_{k, j}(t)=x$ se e somente se $X_{n, j}\left(\theta_{k+1}^{n}(t)\right)=$ $x$. Como $\theta_{k+1}^{n}$ é estritamente crescente, podemos aplicar a Proposição 2.8 para obter que:

$$
\begin{aligned}
X_{n, j}\left(\theta_{k+1}^{n}(t)\right)=x & \\
\Leftrightarrow & \theta_{k+1}^{n}(t) \in \bigcup_{i=1}^{\infty}\left[\theta_{j}^{n}\left(\sigma_{i}^{j, x}-\right), \theta_{j}^{n}\left(\sigma_{i}^{j, x}\right)\right) \\
& \Leftrightarrow \theta_{k+1}^{n}(t) \in \bigcup_{i=1}^{\infty}\left[\theta_{k+1}^{n}\left(\theta_{j}^{k}\left(\sigma_{i}^{j, x}-\right)\right), \theta_{k+1}^{n}\left(\theta_{j}^{k}\left(\sigma_{i}^{j, x}\right)\right)\right) \\
& \Leftrightarrow t \in \bigcup_{i=1}^{\infty}\left[\theta_{j}^{k}\left(\sigma_{i}^{j, x}-\right), \theta_{j}^{k}\left(\sigma_{i}^{j, x}\right)\right) \\
& \Leftrightarrow X_{k, j}(t)=x .
\end{aligned}
$$

Proposição 2.11. Para todo $j<k$, a imagem de $\theta_{j}^{k}$ é o conjunto $\left\{t \geqslant 0: X_{k, j}(t)=\infty\right\}$ q.c. .

Demonstração. Em vez de mostrar que a imagem de $\theta_{j}^{k}$ é igual ao conjunto $\left\{t \geqslant 0: X_{k, j}(t)=\infty\right\}$, vamos mostrar que os complementares dos dois conjuntos são iguais.

Primeiramente tome $t$ seja tal que $X_{k, j}(t)=x<\infty$. Pela Proposição 2.8 temos que existe $i \in \mathbb{N}_{*}$ tal que $t \in\left[\theta_{j}^{k}\left(\sigma_{i}^{j, x}-\right), \theta_{j}^{k}\left(\sigma_{i}^{j, x}\right)\right)$. Como $\theta_{j}^{k}$ é q.c. estritamente crescente, isso implica que $t$ não pertence à imagem de $\theta_{j}^{k}$.

Agora suponha que $t$ não pertença à imagem de $\theta_{j}^{k}$. Como $\theta_{j}^{k}$ é estritamente crescente e $\lim _{r \rightarrow \infty} \theta_{j}^{k}(r)=\infty$ q.c. então existe q.c. um $s$ tal que $t \in\left[\theta_{j}^{k}(s-), \theta_{j}^{k}(s)\right)$. 
Como $s$ é um ponto de descontinuidade de $\theta_{j}^{k}$, a Proposição 2.9 nos diz que $s=\sigma_{i}^{j, x}$ para algum $i, x \in \mathbb{N}_{*}$, então teremos, pela Proposição 2.8 , que $X_{k, j}(t)=x<\infty$.

Proposição 2.12. Quase certamente, para qualquer $j \leqslant k$, vale que $X_{k, j}$ assume um valor finito $e$ constante em qualquer intervalo $\left[\Xi_{k}\left(\sigma_{i}^{k, x}-\right), \Xi_{k}\left(\sigma_{i}^{k, x}\right)\right)$.

Demonstração. O caso $j=k$ é obtido diretamente da construção, dessa forma vamos supor que $j<k$.

Note que, se $t \in\left[\Xi_{k}\left(\sigma_{i}^{k, x}-\right), \Xi_{k}\left(\sigma_{i}^{k, x}\right)\right)$, então $\Xi_{k}^{-1}(t)=\sigma_{i}^{k, x}$. Como, pela construção em $(2.4 \mathrm{~b})$, $X_{k, j}(t)=X_{k-1, j}\left(\Xi_{k}^{-1}(t)\right)=X_{k-1, j}\left(\sigma_{i}^{k, x}\right)$, então $X_{k, j}$ é constante nesse intervalo. Resta mostrar que esse valor também é finito.

Por absurdo suponha que $X_{k, j}(t)=\infty$. A Proposição 2.11 nos diz que $t$ pertence à imagem de $\theta_{j}^{k}$. Como $\theta_{j}^{k}=\Xi_{k} \circ \theta_{j}^{k-1}$ então a imagem de $\theta_{j}^{k}$ está contida na imagem de $\Xi_{k}$. Dessa forma existe um $s$ tal que $\Xi_{k}(s)=t$. Porém isso contradiz a nossa escolha de $t \in\left[\Xi_{k}\left(\sigma_{i}^{k, x}-\right), \Xi_{k}\left(\sigma_{i}^{k, x}\right)\right)$.

Proposição 2.13. Para todo $k$, o conjunto $\left\{t \geqslant 0: \mathbb{P}\left(X_{k}(t) \notin \mathbb{N}_{*}^{k}\right)>0\right\}$ tem medida de Lebesgue nula.

Demonstração. Para $j \leqslant k$, a Proposição 2.11 nos diz que o conjunto $\left\{t \geqslant 0: X_{k, j}(t)=\infty\right\}$ é q.c. a imagem de $\theta_{j}^{k}$. Como esses relógios são definidos por composição, temos que:

$$
\theta_{1}^{k}\left(\mathbb{R}^{+}\right) \subseteq \theta_{2}^{k}\left(\mathbb{R}^{+}\right) \subseteq \ldots \subseteq \theta_{k}^{k}\left(\mathbb{R}^{+}\right)
$$

Portanto $\mathbb{P}\left(X_{k}(t) \notin \mathbb{N}_{*}^{k}\right)=\mathbb{P}\left(t \in \Xi_{k}\left(\mathbb{R}^{+}\right)\right)$. A Proposição 2.5 nos diz que a essa imagem tem medida de Lebesgue nula quase certamente. Dessa forma, usando o Teorema de Fubini obtemos:

$$
\int_{0}^{\infty} \mathbb{P}\left(X_{k}(t) \notin \mathbb{N}_{*}^{k}\right) d t=\int_{0}^{\infty} \mathbb{P}\left(t \in \Xi_{k}\left(\mathbb{R}^{+}\right)\right) d t=\mathbb{E}\left[\int_{0}^{\infty} \mathbb{I}\left\{t \in \Xi_{k}\left(\mathbb{R}^{+}\right)\right\} d t\right]=0 .
$$

Proposição 2.14. Se $\gamma=\left\{\gamma_{k}\left(\left.x\right|_{k}\right): k \in \mathbb{N}_{*},\left.x\right|_{k} \in \mathbb{N}_{*}^{k}\right\}$ são fixados, então $\theta_{1}^{n}$ é um subordinador para cada $n \in \mathbb{N}_{*}$. Além disso, para quase toda realização de $\gamma$ :

$$
\mathbb{E}_{\gamma}\left[\theta_{1}^{n}(t)\right]=t \sum_{\left.x\right|_{n}} \bar{\gamma}_{n}\left(\left.x\right|_{n}\right)
$$

Demonstração. O valor esperado foi calculado na prova da Proposição 2.4. Vamos provar que $\theta_{1}^{n}$ é um subordinador, isso é, um processo estocástico com incrementos estacionários e independentes. O caso $n=1$ é uma consequência direta da independência e estacionariedade dos incrementos de um Processo de Poisson e o fato que as variáveis exponenciais usadas para construir $\theta_{1}^{1}=\Xi_{1}$ são independentes.

Tomando $n \geqslant 2$, note que para $t, s>0$ :

$$
\theta_{1}^{n}(t+s)-\theta_{1}^{n}(t)=\sum_{x} \sum_{i=N^{n, x}\left(\theta_{1}^{n-1}(t)\right)+1}^{N^{n, x}\left(\theta_{1}^{n-1}(t+s)\right)} \gamma_{n}\left(X_{n-1}\left(\sigma_{i}^{n, x}\right) x\right) T_{i}^{n, x}
$$

Fixe $0=t_{0}<t_{1}<\ldots<t_{k}$ e vamos olhar para a distribuição conjunta de $\left(\theta_{1}^{n}\left(t_{i}\right)-\theta_{1}^{n}\left(t_{i-1}\right)\right)_{i=1, \ldots, k}$. 
Variando os valores de $i$, notamos que $\theta_{1}^{n}\left(t_{i}\right)-\theta_{1}^{n}\left(t_{i-1}\right)$, dependem de intervalos disjuntos dos Processos de Poisson $N^{n, x}$, cada um com comprimento $\theta_{1}^{n-1}\left(t_{i}\right)-\theta_{1}^{n-1}\left(t_{i-1}\right)$. Pela hipótese de indução esses comprimentos são independentes e cada um tem a mesma lei que $\theta_{1}^{n-1}\left(t_{i}-t_{i-1}\right)$.

A Proposição 2.11 nos diz que, para todo $t>0, X_{n-1}\left(\theta_{1}^{n-1}(t)\right)=(\infty, \ldots, \infty)$ q.c. . Este instante $\theta_{1}^{n-1}(t)$ é um tempo de renovação, isso é, o que acontece antes dessa renovação é independente do que acontece depois. Isso porque, por construção, a lei de $X_{n-1}$ após uma dessas renovações é a mesma que a lei logo após o instante zero.

$\theta_{1}^{n}\left(t_{i}\right)-\theta_{1}^{n}\left(t_{i-1}\right)$ também depende dos valores de $X_{n-1}(r)$ para $r \in\left(\theta_{1}^{n-1}\left(t_{i-1}\right), \theta_{1}^{n-1}\left(t_{i}\right)\right)$. Ambos os extremos desses intervalos são renovações, então o que acontece à $X_{n-1}$ dentro de um desses intervalos é independente do que acontece em outros intervalos.

Portanto mostramos que $\theta_{1}^{n}\left(t_{i}\right)-\theta_{1}^{n}\left(t_{i-1}\right)$ depende de quantidades que são independentes e cujas leis não dependem do início do intervalo $\left(t_{i-1}\right)$, mas somente do seu comprimento $\left(t_{i}-t_{i-1}\right)$. Portanto $\theta_{1}^{n}$ tem incrementos independentes e estacionários. 


\section{Capítulo 3}

\section{Relógios Limite}

Nesse capítulo vamos construir processos estocásticos $\theta_{k}^{\infty}$, que serão limites, em algum sentido, dos processos $\theta_{k}^{n}$ quando $n \rightarrow \infty$. Esse resultado será melhor especificado e provado no Teorema 4.5 .

Esses processos permitirão, em analogia à Proposição 2.8, definir o Processo K em uma árvore de profundidade infinita.

\subsection{Limites do meio aleatório}

Proposição 3.1. Para cada $\left.x\right|_{k} \in \mathbb{N}_{*}^{k}$, o seguinte limite existe quase certamente:

$$
W\left(\left.x\right|_{k}\right):=\lim _{n \rightarrow \infty} \sum_{\left.y\right|_{n} \in\left[\left.x\right|_{k}\right]_{n}}\left(\frac{\bar{\gamma}_{n}\left(\left.y\right|_{n}\right)}{\bar{\gamma}_{k}\left(\left.y\right|_{k}\right)}\right)^{\alpha_{n+1}} .
$$

Além disso, $W\left(\left.x\right|_{k}\right)$ tem uma distribuição $\alpha_{k+1}$-estável, com transformada de Laplace dada por:

$$
\mathbb{E}\left[e^{-\lambda W\left(\left.x\right|_{k}\right)}\right]=\exp \left\{-\lambda^{\alpha_{k+1}}\right\} .
$$

Demonstração. Sem perda de generalidade, vamos tomar $k=0$ e definir as variáveis aleatórias $Z_{n}(\lambda)$, para $\lambda>0$ :

$$
Z_{n}(\lambda):=\exp \left\{-\sum_{\left.y\right|_{n} \in \mathbb{N}_{*}^{n}}\left(\lambda \bar{\gamma}_{n}\left(\left.y\right|_{n}\right)\right)^{\alpha_{n+1}}\right\}
$$

Vamos mostrar que essa sequência de variáveis aleatórias, para cada $\lambda>0$ fixado, formam um martingal com relação à uma filtração $\left(\mathcal{F}_{n}\right)$, onde $\mathcal{F}_{n}$ é a $\sigma$-algebra gerada por todos os Processos Pontuais de Poisson $\left\{\gamma_{j}\left(\left.x\right|_{j}\right): j \leqslant n,\left.x\right|_{j} \in \mathbb{N}_{*}^{j}\right\}$. Isso será feito novamente com o Teorema de Campbell: 


$$
\begin{aligned}
\mathbb{E}\left(Z_{n+1}(\lambda) \mid \mathcal{F}_{n}\right) & =\mathbb{E}\left[\exp \left\{-\sum_{\left.y\right|_{n+1}}\left(\lambda \bar{\gamma}_{n+1}\left(\left.y\right|_{n+1}\right)\right)^{\alpha_{n+2}}\right\} \mid \mathcal{F}_{n}\right] \\
& =\prod_{\left.y\right|_{n}} \mathbb{E}\left[\exp \left\{-\left(\lambda \bar{\gamma}_{n}\left(\left.y\right|_{n}\right)\right)^{\alpha_{n+2}} \sum_{y_{n+1}} \gamma_{n+1}\left(\left.y\right|_{n+1}\right)^{\alpha_{n+2}}\right\} \mid \mathcal{F}_{n}\right] \\
& =\prod_{\left.y\right|_{n}} \exp \left\{-\int_{0}^{\infty}\left(1-e^{\left.\left.-\left(\lambda \bar{\gamma}_{n}\left(\left.y\right|_{n}\right)\right)^{\alpha_{n+2}} t^{\alpha_{n+2}}\right) \mu_{n+1}(d t)\right\}}\right.\right. \\
& =\prod_{\left.y\right|_{n}} \exp \left\{-\left(\lambda \bar{\gamma}_{n}\left(\left.y\right|_{n}\right)\right)^{\alpha_{n+1}}\right\} \\
& =\exp \left\{-\sum_{\left.y\right|_{n}}\left(\lambda \bar{\gamma}_{n}\left(\left.y\right|_{n}\right)\right)^{\alpha_{n+1}}\right\}=Z_{n}(\lambda) .
\end{aligned}
$$

Já que $\left(Z_{n}(1)\right)_{n}$ é um martingal positivo, podemos aplicar um teorema de convergência de martingais - por exemplo, Teorema 5.2.9 de Durrett [Dur10] - para concluir que $Z_{n}(1)$ converge q.c. . Como $Z_{n}(1)$ converge, então o seu expoente deve convergir também.

A segunda afirmação dessa proposição pode ser obtida ao calcular explicitamente a transformada de Laplace de $W\left(\left.x\right|_{k}\right)$ :

$$
\begin{aligned}
\mathbb{E}\left[\exp \left\{-\lambda \sum_{\left.y\right|_{n}} \bar{\gamma}_{n}\left(\left.y\right|_{n}\right)^{\alpha_{n+1}}\right\}\right] & =\mathbb{E}\left[Z_{n}\left(\lambda^{1 / \alpha_{n+1}}\right)\right] \\
& =\mathbb{E}\left[Z_{1}\left(\lambda^{1 / \alpha_{n+1}}\right)\right] \\
& =\exp \left\{-\lambda^{\alpha_{1} / \alpha_{n+1}}\right\} \stackrel{n \rightarrow \infty}{\longrightarrow} \exp \left\{-\lambda^{\alpha_{1}}\right\} .
\end{aligned}
$$

Observação 3.2. Note que nossa escolha para as constantes $c_{k}$ feita em (2.1) foi vital para essa demonstração. Fizemos aquela escolha explicitamente para tornar $Z_{n}(\lambda)$ um martingal.

Proposição 3.3. A família de variáveis aleatórias $\left\{W\left(\left.x\right|_{k}\right):\left.x\right|_{k} \in \bigcup_{j=0}^{\infty} \mathbb{N}_{*}^{j}\right\}$ satisfaz uma lei de composição. Para cada $\left.x\right|_{k} \in \mathbb{N}_{*}^{k}$ fixado vale que q.c. :

$$
W\left(\left.x\right|_{k}\right)=\sum_{x_{k+1}} \gamma_{k+1}\left(\left.x\right|_{k+1}\right) W\left(\left.x\right|_{k+1}\right)
$$

Demonstração. Fixe uma realização tal que (3.1) seja verdade para todo $\left.x\right|_{k} \in \cup_{j=1}^{\infty} \mathbb{N}_{*}^{j}$ e fixe um 
$\epsilon>0$ arbitrário, então:

$$
\begin{aligned}
W\left(\left.x\right|_{k}\right) & =\lim _{n \rightarrow \infty} \sum_{\left.y\right|_{n} \in\left[\left.x\right|_{k}\right]_{n}}\left(\frac{\bar{\gamma}_{n}\left(\left.y\right|_{n}\right)}{\bar{\gamma}_{k}\left(\left.x\right|_{k}\right)}\right)^{\alpha_{n+1}} \\
& =\lim _{n \rightarrow \infty} \sum_{x_{k+1}}\left(\gamma_{k+1}\left(\left.x\right|_{k+1}\right)\right)^{\alpha_{n+1}} \sum_{y_{n} \in\left[\left.x\right|_{k+1}\right]_{n}}\left(\frac{\bar{\gamma}_{n}\left(\left.y\right|_{n}\right)}{\bar{\gamma}_{k+1}\left(\left.x\right|_{k+1}\right)}\right)^{\alpha_{n+1}} \\
& =\lim _{n \rightarrow \infty} \sum_{\substack{x_{k+1}: \\
\gamma_{k+1}\left(\left.x\right|_{k+1}\right)>\epsilon}}\left(\gamma_{k+1}\left(\left.x\right|_{k+1}\right)\right)^{\alpha_{n+1}} \sum_{y_{n} \in\left[\left.x\right|_{k+1}\right]_{n}}\left(\frac{\bar{\gamma}_{n}\left(\left.y\right|_{n}\right)}{\bar{\gamma}_{k+1}\left(\left.x\right|_{k+1}\right)}\right)^{\alpha_{n+1}} \\
& +\lim _{n \rightarrow \infty} \sum_{\substack{x_{k+1}: \\
\gamma_{k+1}\left(\left.x\right|_{k+1} \leqslant \epsilon\right.}}\left(\gamma_{k+1}\left(\left.x\right|_{k+1}\right)\right)^{\alpha_{n+1}} \sum_{y_{n} \in\left[\left.x\right|_{k+1}\right]_{n}}\left(\frac{\bar{\gamma}_{n}\left(\left.y\right|_{n}\right)}{\bar{\gamma}_{k+1}\left(\left.x\right|_{k+1}\right)}\right)^{\alpha_{n+1}} .
\end{aligned}
$$

Vamos tratar esses dois termos separadamente e mostrar que os seus respectivos limites existem em probabilidade. Em (3.5), já que a soma exterior é finita e $\alpha_{n} \rightarrow 1$ quando $n \rightarrow \infty$, então:

$$
\begin{aligned}
(3.5) & =\sum_{\substack{x_{k+1}: \\
\gamma_{k+1}\left(\left.x\right|_{k+1}\right)>\epsilon}} \gamma_{k+1}\left(\left.x\right|_{k+1}\right) \lim _{n \rightarrow \infty} \sum_{y_{n} \in\left[\left.x\right|_{k+1}\right]_{n}}\left(\frac{\bar{\gamma}_{n}\left(\left.y\right|_{n}\right)}{\bar{\gamma}_{k}\left(\left.x\right|_{k+1}\right)}\right)^{\alpha_{n+1}} \\
& =\sum_{\substack{x_{k+1}: \\
\gamma_{k+1}\left(\left.x\right|_{k+1}\right)>\epsilon}} \gamma_{k+1}\left(\left.x\right|_{k+1}\right) W\left(\left.x\right|_{k+1}\right) \stackrel{\epsilon \rightarrow 0}{\longrightarrow} \sum_{x_{k+1}} \gamma_{k+1}\left(\left.x\right|_{k+1}\right) W\left(\left.x\right|_{k+1}\right) .
\end{aligned}
$$

Tome $\mathcal{G}_{\left.x\right|_{k}}$ a $\sigma$-álgebra gerada pelos Processos Pontuais de Poisson $\left\{\gamma_{k+1}\left(\left.x\right|_{k+1}\right): x_{k+1} \in \mathbb{N}_{*}\right\}$. Usando (3.3), podemos calcular a transformada de Laplace de (3.6) como:

$$
\begin{aligned}
& \mathbb{E}\left[\exp \left\{-\lambda \sum_{\substack{x_{k+1}: \\
\gamma_{k+1}\left(\left.x\right|_{k+1}\right) \leqslant \epsilon}}\left(\gamma_{k+1}\left(\left.x\right|_{k+1}\right)\right)^{\alpha_{n+1}} \sum_{y_{n} \in\left[\left.x\right|_{k+1}\right]_{n}}\left(\frac{\bar{\gamma}_{n}\left(\left.y\right|_{n}\right)}{\bar{\gamma}_{k+1}\left(\left.x\right|_{k+1}\right)}\right)^{\alpha_{n+1}}\right\}\right] \\
& =\mathbb{E}\left[\prod_{\substack{x_{k+1}: \\
\gamma_{k+1}\left(\left.x\right|_{k+1}\right) \leqslant \epsilon}} \mathbb{E}\left[\exp \left\{-\lambda\left(\gamma_{k+1}\left(\left.x\right|_{k+1}\right)\right)^{\alpha_{n+1}} \sum_{y_{n} \in\left[\left.x\right|_{k+1}\right]_{n}}\left(\frac{\bar{\gamma}_{n}\left(\left.y\right|_{n}\right)}{\bar{\gamma}_{k+1}\left(\left.x\right|_{k+1}\right)}\right)^{\alpha_{n+1}}\right\} \mid \mathcal{G}_{\left.x\right|_{k}}\right]\right] \\
& =\mathbb{E}\left[\prod_{\substack{x_{k+1}: \\
\gamma_{k+1}\left(\left.x\right|_{k+1}\right) \leqslant \epsilon}} \exp \left\{-\left(\lambda\left(\gamma_{k+1}\left(\left.x\right|_{k+1}\right)\right)^{\alpha_{n+1}}\right)^{\frac{\alpha_{k+2}}{\alpha_{n+1}}}\right\}\right] \\
& \stackrel{n \rightarrow \infty}{\longrightarrow} \mathbb{E}\left[\exp \left\{-\lambda^{\alpha_{k+2}} \sum_{\substack{x_{k+1}: \\
\gamma_{k+1}\left(\left.x\right|_{k+1}\right) \leqslant \epsilon}}\left(\gamma_{k+1}\left(\left.x\right|_{k+1}\right)\right)^{\alpha_{k+2}}\right\}\right] \\
& =\exp \left\{-\int_{0}^{\epsilon}\left(1-e^{-\lambda^{\alpha_{k+2}} x^{\alpha_{k+2}}}\right) \mu_{k+1}(d x)\right\} \stackrel{\epsilon \rightarrow 0}{\longrightarrow} 1 \text {. }
\end{aligned}
$$

Usamos o Teorema de Campbell para a última passagem. Com esse resultado obtemos que (3.6) converge em probabilidade para 0 quando $\epsilon \rightarrow 0$.

Finalmente, note que mostramos que $(3.5)+(3.6)$ converge em probabilidade tanto para $W\left(\left.x\right|_{k}\right)$ quanto para $\sum_{x_{k+1}} \gamma_{k+1}\left(\left.x\right|_{k+1}\right) W\left(\left.x\right|_{k+1}\right)$ quando $\epsilon \rightarrow 0$. Isso implica que estas duas últimas quan- 
tidades são iguais quase certamente.

\subsection{Relógios modificados}

Nosso objetivo nessa seção é definir e provar a existência dos processos $\theta_{k}^{\infty}$. Provaremos no próximo capítulo que esses processos são o limite dos processos $\theta_{k}^{n}$ quando $n \rightarrow \infty$. Eles serão usados para definir o Processo K em uma árvore de profundidade infinita.

Com esse objetivo em mente, vamos introduzir uma perturbação nos relógios $\theta_{k}^{n}$ utilizando as variáveis $W\left(\left.x\right|_{k}\right)$ introduzidas na Seção 3.1. Iremos provar no próximo capítulo que essa perturbação não afeta o limite.

Essa perturbação é feita com o objetivo de transformar os relógios em martingais, o que será provado no Teorema 3.6. Isso é o que permitirá estabelecer limites quase certos desses relógios modificados.

Definição 3.1. Definimos os relógios ajustados como:

$$
\begin{aligned}
\widetilde{\Xi}_{j}(t) & :=\sum_{x \in \mathbb{N}_{*}} \sum_{i=1}^{N^{j, x}(t)} W\left(X_{j-1}\left(\sigma_{i}^{j, x}\right) x\right) \gamma_{j}\left(X_{j-1}\left(\sigma_{i}^{j, x}\right) x\right) T_{i}^{j, x} \\
\widetilde{\theta}_{k}^{n} & :=\widetilde{\Xi}_{n} \circ \Xi_{n-1} \circ \cdots \circ \Xi_{k} .
\end{aligned}
$$

Também representamos o tempo que o Processo $K$ em uma árvore de profundidade $k, X_{k}$, passa em um estado $\left.x\right|_{k}$ até o instante $t$ por $L_{k}\left(\left.x\right|_{k}, t\right)$, isso é:

$$
L_{k}\left(\left.x\right|_{k}, t\right)=\int_{0}^{t} \mathbb{I}\left\{X_{k}(s)=\left.x\right|_{k}\right\} d s .
$$

Se fixarmos o meio aleatório $\left\{\gamma_{k}\left(\left.x\right|_{k}\right): k \in \mathbb{N}_{*},\left.x\right|_{k} \in \mathbb{N}_{*}^{k}\right\}$, então $\theta_{1}^{n}$ é um subordinador (Proposição 2.14). Porém $\theta_{k}^{n}$, com $k>1$, não é. Isso cria várias complicações técnicas. Para contorna-las iremos quebrar $\theta_{k}^{n}$ em uma soma de funções que são subordinadores.

Definição 3.2. Para $n>k \geqslant 1$ fixados, denote por $\nu_{k+1}^{n}$ a única medida aleatória nos borelianos de $[0, \infty)$ que satisfaz $\nu_{k+1}^{n}([0, t])=\theta_{k+1}^{n}(t)$. Para um $\left.x\right|_{k} \in \mathbb{N}_{*}^{k}$ fixado, definimos:

$$
\theta_{\left.x\right|_{k}}^{n}(t):=\nu_{k+1}^{n}\left(\left\{s \in[0, \infty): L_{k}\left(\left.x\right|_{k}, s\right) \leqslant t, X_{k}(s)=\left.x\right|_{k}\right\}\right) .
$$

A Figura 3.1 ilustra essa definição. Os intervalos marcados na abscissa da Figura 3.1a são aqueles onde $X_{k}=\left.x\right|_{k}$.

Proposição 3.4. A lei de $\theta_{\left.x\right|_{k}}^{n}$ é a mesma que a lei de $\theta_{1}^{n-k}$, com os indices dos $\alpha$ transladados. Isso é, $\theta_{\left.x\right|_{k}}^{n}$ têm a mesma lei que $\hat{\theta}_{1}^{n-k+1}$, onde este último processo é construído da mesma forma que $\theta_{1}^{n-k}$, porém tomando $\left(\widehat{\alpha}_{i}\right)_{i}$ como parâmetros, onde $\widehat{\alpha}_{i}=\alpha_{i+k}$ para $i=1,2, \ldots$.

Vale ainda que os processos $\theta_{\left.x\right|_{k}}^{n}$ são independentes para um $n$ fixado e variando $\left.x\right|_{k}$. Além disso a seguinte igualdade é verdade quase certamente:

$$
\theta_{k+1}^{n}(t)=\sum_{\left.x\right|_{k}} \theta_{\left.x\right|_{k}}^{n}\left(L_{k}\left(\left.x\right|_{k}, t\right)\right)
$$




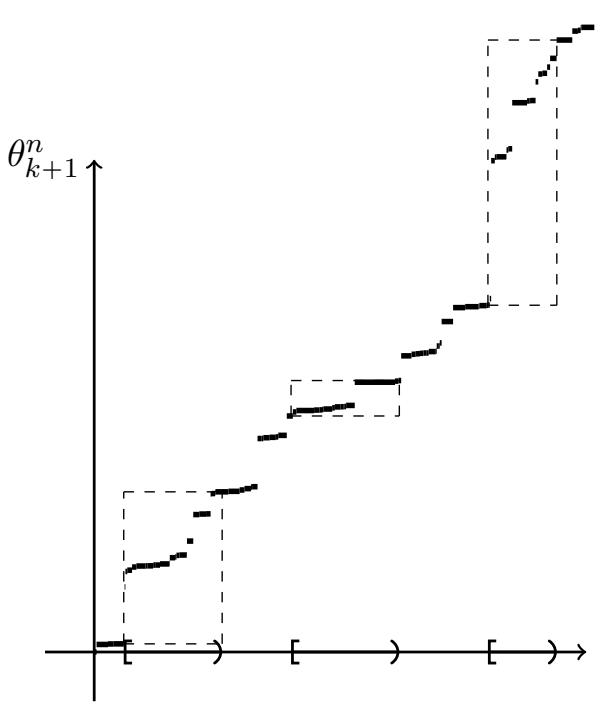

(a) Uma realização de $\theta_{k+1}^{n}$

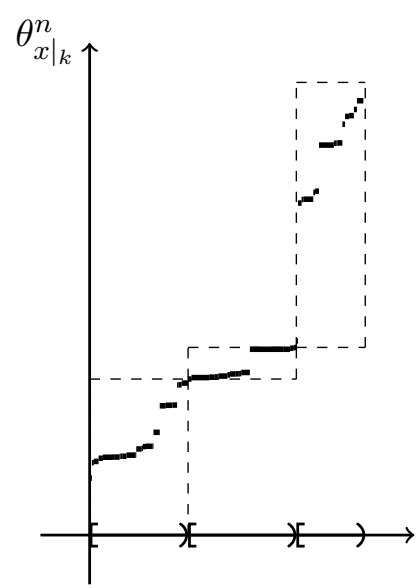

(b) Construindo $\theta_{\left.x\right|_{k}}^{n}$ a partir de $\theta_{k+1}^{n}$

Figura 3.1: Construção de $\theta_{\left.x\right|_{k}}^{n}$

Demonstração. Tomemos $\nu_{k+1}^{n}$ como na Definição 3.2. Como uma medida é $\sigma$-aditiva, é verdade que:

$$
\begin{aligned}
\sum_{\left.x\right|_{k}} \theta_{\left.x\right|_{k}}^{n}\left(L_{k}\left(\left.x\right|_{k}, t\right)\right) & =\sum_{\left.x\right|_{k}} \nu_{k+1}^{n}\left(\left\{s \in[0, \infty): L_{k}\left(\left.x\right|_{k}, s\right) \leqslant L_{k}\left(\left.x\right|_{k}, t\right), X_{k}(s)=\left.x\right|_{k}\right\}\right) \\
& =\sum_{\left.x\right|_{k}} \nu_{k+1}^{n}\left(\left\{s \in[0, t]: X_{k}(s)=\left.x\right|_{k}\right\}\right) \\
& =\nu_{k+1}^{n}\left(\left\{s \in[0, t]: X_{k}(s) \in \mathbb{N}_{*}^{k}\right\}\right) .
\end{aligned}
$$

Por construção, $\theta_{k+1}^{n}$ é uma função escada e seus incrementos ocorrem q.c. no conjunto $\left\{\sigma_{i}^{k+1, x}\right.$ : $\left.i, x \in \mathbb{N}_{*}\right\}$ (Proposição 2.9). A Proposição 2.12 nos diz ainda que $X_{k}$ têm todas as coordenadas finitas nesses pontos, dessa forma obtemos que $(3.9)$ vale $\nu_{k+1}^{n}([0, t])=\theta_{k+1}^{n}(t)$.

Fixado um $\left.x\right|_{k} \in \mathbb{N}_{*}^{k}$, vamos provar que $\theta_{\left.x\right|_{k}}^{n}$ tem a mesma lei que $\theta_{1}^{n-k}$, com os índices $\alpha_{i}$ transladados, por indução em $n$. Vamos omitir a prova da base da indução, o caso $n=k+1$, porque ele pode ser feito de maneira análoga ao passo da indução. Como $\theta_{k+1}^{n}=\Xi_{n} \circ \theta_{k+1}^{n-1}$ :

$$
\theta_{k+1}^{n}(t)=\sum_{x \in \mathbb{N}_{*}} \sum_{i=1}^{N^{n, x}\left(\theta_{k+1}^{n-1}(t)\right)} \gamma_{n}\left(X_{n-1}\left(\sigma_{i}^{n, x}\right) x\right) T_{i}^{n, x}
$$

Quando formos construir $\theta_{\left.x\right|_{k}}^{n}$, um incremento em um ponto $\sigma_{i}^{n, x}$ será considerado se ele corresponder a um $s$ onde $X_{k}(s)=\left.x\right|_{k}$ e $\theta_{k+1}^{n-1}$ "leve" $s$ à $\sigma_{i}^{n, x}$. Isso é, ele será considerado se existir um $s$ tal que $\sigma_{i}^{n, x} \in\left[\theta_{k+1}^{n-1}(s-), \theta_{k+1}^{n-1}(s)\right)$ e $X_{k}(s)=\left.x\right|_{k}$.

Se denotarmos por $g$ a inversa generalizada de $\theta_{k+1}^{n-1}$, vale que $g\left(\sigma_{i}^{n, x}\right)=s$. Utilizando a Proposição 2.10 , temos que $X_{k}(s)$ é igual às primeiras $k$ coordenadas de $X_{n-1}\left(\theta_{k+1}^{n-1}(s)\right)$. Dessa forma, se 
denotarmos por $\left.X_{n-1}\right|_{k}$ as primeiras $k$ coordenadas de $X_{n-1}$, então:

$$
\begin{aligned}
\theta_{\left.x\right|_{k}}^{n}(t) & =\sum_{x \in \mathbb{N}_{*}} \sum_{i=1}^{N^{n, x}\left(L_{k}^{-1}\left(\left.x\right|_{k}, \theta_{k+1}^{n-1}(t)\right)\right)} \mathbb{I}\left\{X_{k}\left(g\left(\sigma_{i}^{n, x}\right)\right)=\left.x\right|_{k}\right\} \gamma_{n}\left(X_{n-1}\left(\sigma_{i}^{n, x}\right) x\right) T_{i}^{n, x} \\
& =\sum_{x \in \mathbb{N}_{*}} \sum_{i=1}^{N^{n, x}\left(L_{k}^{-1}\left(\left.x\right|_{k}, \theta_{k+1}^{n-1}(t)\right)\right)} \mathbb{I}\left\{\left.X_{n-1}\right|_{k}\left(\theta_{k+1}^{n-1}\left(g\left(\sigma_{i}^{n, x}\right)\right)\right)=\left.x\right|_{k}\right\} \gamma_{n}\left(X_{n-1}\left(\sigma_{i}^{n, x}\right) x\right) T_{i}^{n, x} \\
& =\sum_{x \in \mathbb{N}_{*}} \sum_{i=1}^{N^{n, x}\left(L_{k}^{-1}\left(\left.x\right|_{k}, \theta_{k+1}^{n-1}(t)\right)\right)} \mathbb{I}\left\{\left.X_{n-1}\right|_{k}\left(\sigma_{i}^{n, x}\right)=\left.x\right|_{k}\right\} \gamma_{n}\left(X_{n-1}\left(\sigma_{i}^{n, x}\right) x\right) T_{i}^{n, x} .
\end{aligned}
$$

Dessa forma somamos somente sobre os pontos onde $\left.X_{n-1}\right|_{k}=\left.x\right|_{k}$, ficando essas coordenadas sempre constantes e as coordenadas seguintes variando.

Para observar a independência, note que ao variar $\left.x\right|_{k}$ então $\theta_{\left.x\right|_{k}}^{n}$ irá depender de regiões disjuntas nos processos de Poisson que os definem, sendo portanto independentes.

Proposição 3.5. Para $n>k \geqslant 0$, as transformadas de Laplace de $\theta_{k+1}^{n}(t)$ e $\widetilde{\theta}_{k+1}^{n}(t)$ podem ser calculadas como:

$$
\begin{aligned}
\mathbb{E}\left[\exp \left\{-\lambda \theta_{k+1}^{n}(t)\right\}\right] & =\mathbb{E}\left[\exp \left\{-\sum_{\left.x\right|_{k} \in \mathbb{N}_{*}^{k}} L_{k}\left(\left.x\right|_{k}, t\right) \sum_{x_{k+1}} h_{\left.x\right|_{k+1}}\left(\cdots \sum_{x_{n}} h_{\left.x\right|_{n}}(\lambda) \cdots\right)\right\}\right] \\
\mathbb{E}\left[\exp \left\{-\lambda \widetilde{\theta}_{k+1}^{n}(t)\right\}\right] & =\mathbb{E}\left[\exp \left\{-\sum_{\left.x\right|_{k} \in \mathbb{N}_{*}^{k}} L_{k}\left(\left.x\right|_{k}, t\right) \sum_{x_{k+1}} h_{\left.x\right|_{k+1}}\left(\cdots \sum_{x_{n}} h_{\left.x\right|_{n}}\left(\lambda W\left(\left.x\right|_{n}\right)\right) \cdots\right)\right\}\right] \\
h_{\left.x\right|_{k}}(\lambda) & :=\frac{\lambda \gamma_{k}\left(\left.x\right|_{k}\right)}{1+\lambda \gamma_{k}\left(\left.x\right|_{k}\right)}
\end{aligned}
$$

Aqui adotamos a convenção $\mathbb{N}_{*}^{0}=\{\varnothing\}$ e $L_{0}(\varnothing, t)=t$.

Demonstração. Tome $\mathcal{F}_{n}$ como a $\sigma$-álgebra gerada por todas as variáveis aleatórias até o nível $n$ e tome $\left\{Z_{\left.x\right|_{n}}:\left.x\right|_{n} \in \mathbb{N}_{*}^{n}\right\}$ uma família arbitrária de variáveis aleatórias positivas e independentes. Vamos supor que essa família seja independente de $\mathcal{F}_{n}$.

Vamos começar provando que:

$$
\mathbb{E}\left[\exp \left\{-\sum_{\left.x\right|_{n}} Z_{\left.x\right|_{n}} L_{n}\left(\left.x\right|_{n}, \theta_{1}^{n}(t)\right)\right\}\right]=\mathbb{E}\left[\exp \left\{-t \sum_{x_{1}} h_{\left.x\right|_{1}}\left(\cdots \sum_{x_{n}} h_{\left.x\right|_{n}}\left(Z_{\left.x\right|_{n}}\right) \cdots\right)\right\}\right] .
$$

O caso $k=0$ dessa Proposição será obtido ao tomar $Z_{\left.x\right|_{n}}=\lambda$ e $Z_{\left.x\right|_{n}}=\lambda W\left(\left.x\right|_{n}\right)$ para $\theta_{1}^{n}$ e $\tilde{\theta}_{1}^{n}$ respectivamente.

Note que, pela maneira como o Processo K foi construído em (2.4b), e o fato de que um Processo 
de Poisson tem incrementos independentes e estacionários, então:

$$
\begin{aligned}
L_{n}\left(\left.x\right|_{n}, \Xi_{n}(t)\right) & =\sum_{i=1}^{N^{n, x_{n}}(t)} \mathbb{I}\left\{X_{n-1}\left(\sigma_{i}^{n, x_{n}}\right)=\left.x\right|_{n-1}\right\} \gamma_{n}\left(\left.x\right|_{n}\right) T_{i}^{n, x_{n}} \\
& \stackrel{\mathcal{D}}{=} \sum_{i=1}^{N^{n, x_{n}}\left(L_{n-1}\left(\left.x\right|_{n-1}, t\right)\right)} \gamma_{n}\left(\left.x\right|_{n}\right) T_{i}^{n, x_{n}} .
\end{aligned}
$$

Tomando $\mathcal{G}_{n}:=\sigma\left(\mathcal{F}_{n-1}, Z_{\left.x\right|_{n}}:\left.x\right|_{n} \in \mathbb{N}_{*}^{n}\right)$, podemos calcular:

$$
\begin{aligned}
\mathbb{E}\left[e^{-Z_{x \mid n} L_{n}\left(\left.x\right|_{n}, \theta_{1}^{n}(t)\right)} \mid \mathcal{G}_{n}\right] & =\mathbb{E}\left[\exp \left\{-Z_{\left.x\right|_{n}} \sum_{i=1}^{N^{n, x_{n}}\left(L_{n-1}\left(\left.x\right|_{n-1}, \theta_{1}^{n-1}(t)\right)\right)} \gamma_{n}\left(\left.x\right|_{n}\right) T_{i}^{n, x_{n}}\right\} \mid \mathcal{G}_{n}\right] \\
& =\exp \left\{-L_{n-1}\left(\left.x\right|_{n-1}, \theta_{1}^{n-1}(t)\right) h_{\left.x\right|_{n}}\left(Z_{\left.x\right|_{n}}\right)\right\}
\end{aligned}
$$

Agora vamos provar (3.11) por indução em $n$. A base da indução, $n=1$, é obtida por conferência direta. Supondo que o resultado é válido para $n-1$, podemos escrever:

$$
\begin{aligned}
\mathbb{E}\left[\exp \left\{-\sum_{\left.x\right|_{n}} Z_{\left.x\right|_{n}} L_{n}\left(\left.x\right|_{n}, \theta_{1}^{n}(t)\right)\right\}\right. & =\mathbb{E}\left[\mathbb{E}\left[\exp \left\{-\sum_{\left.x\right|_{n}} Z_{\left.x\right|_{n}} L_{n}\left(\left.x\right|_{n}, \theta_{1}^{n}(t)\right)\right\} \mid \mathcal{G}_{n}\right]\right] \\
& =\mathbb{E}\left[\prod_{\left.x\right|_{n}} \mathbb{E}\left[\exp \left\{-Z_{\left.x\right|_{n}} L_{n}\left(\left.x\right|_{n}, \theta_{1}^{n}(t)\right)\right\} \mid \mathcal{G}_{n}\right]\right] \\
& =\mathbb{E}\left[\exp \left\{-\sum_{\left.x\right|_{n-1}} L_{n}\left(\left.x\right|_{n-1}, \theta_{1}^{n-1}(t)\right) \sum_{x_{n}} h_{\left.x\right|_{n}}\left(Z_{\left.x\right|_{n}}\right)\right\}\right] .
\end{aligned}
$$

Tomando $Z_{\left.x\right|_{n-1}}:=\sum_{x_{n}} h_{\left.x\right|_{n}}\left(Z_{\left.x\right|_{n}}\right)$, podemos aplicar a hipótese de indução, obtendo (3.11), de onde concluímos o caso $k=0$.

Para o caso geral vamos usar a Proposição 3.4 para escrever:

$$
\begin{aligned}
\mathbb{E}\left[e^{-\lambda \theta_{k+1}^{n}(t)}\right] & =\mathbb{E}\left[\exp \left\{-\lambda \sum_{\left.x\right|_{k}} \theta_{\left.x\right|_{k}}^{n}\left(L_{k}\left(\left.x\right|_{k}, t\right)\right)\right\}\right] \\
& =\mathbb{E}\left[\prod_{\left.x\right|_{k}} \mathbb{E}\left[\exp \left\{-\lambda \theta_{\left.x\right|_{k}}^{n}\left(L_{k}\left(\left.x\right|_{k}, t\right)\right)\right\} \mid \mathcal{F}_{k}\right]\right] \\
& =\mathbb{E}\left[\exp \left\{-\sum_{\left.x\right|_{k}} L_{k}\left(\left.x\right|_{k}, t\right) Z_{\left.x\right|_{k}}^{n}\right\}\right] .
\end{aligned}
$$

O resultado correspondente para $\widetilde{\theta}_{k+1}^{n}$ pode ser provado de forma análoga.

Teorema 3.6. Para cada $k \in \mathbb{N}_{*}$, existe quase certamente um processo estocástico $\theta_{k}^{\infty}$ que é não decrescente e contínuo a direita, tal que $\lim _{n \rightarrow \infty} \tilde{\theta}_{k}^{n}(t)=\theta_{k}^{\infty}(t)$ q.c. para todo $t \in D_{k}$, onde $D_{k}$ é um conjunto determinístico, denso em $(0, \infty)$ e enumerável.

Além disso, para todo $t>0, \theta_{k}^{\infty}(t)$ é q.c. finito e se fixarmos o meio aleatório $\gamma=\left\{\gamma_{k}\left(\left.x\right|_{k}\right)\right\}$ então $\mathbb{E}_{\gamma}\left[\theta_{k}^{\infty}(t)\right]<\infty$ q.c. . 
Demonstração. Vamos fixar as variáveis $\left\{\gamma_{k}\left(\left.x\right|_{k}\right):\left.x\right|_{k} \in \mathbb{N}_{*}^{k}, k \in \mathbb{N}\right\}$ e provar o resultado para quase todas as escolhas desse meio aleatório. Vamos denotar por $\mathcal{G}_{k}$ a $\sigma$-álgebra gerada por todos os Processos de Poisson e variáveis exponenciais até a profundidade $k$.

Vamos adotar $D_{1}=\mathbb{Q} \cap(0, \infty)$. Para $k>1$, a Proposição 2.13 nos diz que o conjunto $\{t \geqslant$ $\left.0: \mathbb{P}\left(X_{k-1}(t) \notin \mathbb{N}_{*}^{k-1}\right)=0\right\}$ têm medida de Lebesgue total. Dessa forma esse conjunto é denso em $(0, \infty)$. Tomemos $D_{k}$ um subconjunto denso enumerável desse conjunto.

Usando da definição em (3.7), podemos dividir as regiões onde $\widetilde{\Xi}_{k+1}$ usa o meio aleatório na subárvore de cada $\left.x\right|_{k}$, obtendo:

$$
\begin{aligned}
\mathbb{E}_{\gamma}\left[\widetilde{\Xi}_{k+1}(t) \mid \mathcal{G}_{k}\right] & =\sum_{\left.x\right|_{k}} L_{k}\left(\left.x\right|_{k}, t\right) \sum_{x_{k+1}} W\left(\left.x\right|_{k+1}\right) \gamma_{k+1}\left(\left.x\right|_{k+1}\right) \\
& =\sum_{\left.x\right|_{k}} L_{k}\left(\left.x\right|_{k}, t\right) W\left(\left.x\right|_{k}\right) .
\end{aligned}
$$

Reescrevendo a definição em (3.7), obtemos:

$$
\begin{aligned}
\widetilde{\Xi}_{n}(t) & =\sum_{x_{n} \in \mathbb{N}_{*}} \sum_{i=1}^{N^{n, x_{n}}(t)} W\left(X_{n-1}\left(\sigma_{i}^{n, x_{n}}\right) x_{n}\right) \gamma_{n}\left(X_{n-1}\left(\sigma_{i}^{n, x_{n}}\right) x_{n}\right) T_{i}^{n, x_{n}} \\
& =\sum_{\left.x\right|_{n} \in \mathbb{N}_{*}^{n}} \sum_{i=1}^{N^{n, x_{n}}(t)} W\left(\left.x\right|_{n}\right) \gamma_{n}\left(\left.x\right|_{n}\right) \mathbb{I}\left\{X_{n-1}\left(\sigma_{i}^{n, x_{n}}\right)=\left.x\right|_{n-1}\right\} T_{i}^{n, x_{n}} \\
& =\sum_{\left.x\right|_{n} \in \mathbb{N}_{*}^{n}} W\left(\left.x\right|_{n}\right) L_{n}\left(\left.x\right|_{n}, \Xi_{n}(t)\right) .
\end{aligned}
$$

Finalmente, para $n>k$ :

$$
\begin{aligned}
\mathbb{E}_{\gamma}\left[\widetilde{\theta}_{k}^{n+1}(t) \mid \mathcal{G}_{n}\right] & =\mathbb{E}_{\gamma}\left[\widetilde{\Xi}_{n+1}\left(\theta_{k}^{n}(t)\right) \mid \mathcal{G}_{n}\right] \\
& =\sum_{\left.x\right|_{n}} L_{n}\left(\left.x\right|_{n}, \theta_{k}^{n}(t)\right) W\left(\left.x\right|_{n}\right) \\
& =\widetilde{\theta}_{k}^{n}(t) .
\end{aligned}
$$

Com isso mostramos que $\left(\widetilde{\theta}_{k}^{n}(t)\right)_{n>k}$ é um martingal com relação à filtração $\left(\mathcal{G}_{n}\right)_{n}$ para cada $t \geqslant 0$ fixo. Assim podemos novamente utilizar um teorema de convergência de martingais, como o Teorema 5.2.9 de Durrett [Dur10] para concluir que $\lim _{n \rightarrow \infty} \widetilde{\theta}_{k}^{n}(t)$ existe e é finito q.c. para cada $t \geqslant 0$ fixado. Esse Teorema ainda nos diz que, quando o meio aleatório $\left\{\gamma_{k}\left(\left.x\right|_{k}\right)\right\}$ está fixado, então:

$$
\mathbb{E}_{\gamma}\left(\lim _{n \rightarrow \infty} \widetilde{\theta}_{k}^{n}(t)\right) \leqslant \liminf _{n \rightarrow \infty} \mathbb{E}_{\gamma}\left(\widetilde{\theta}_{k}^{n}(t)\right)=\mathbb{E}_{\gamma}\left(\widetilde{\theta}_{k}^{k+1}(t)\right)<\infty
$$

Tomemos esses limites $\theta_{k}^{\infty}(t):=\lim _{n \rightarrow \infty} \widetilde{\theta}_{k}^{n}(t)$ para todo $t \in D_{k}$. Já que cada $\widetilde{\theta}_{k}^{n}$ é monótono, então o limite será monótono também.

Agora podemos definir $\theta_{k}^{\infty}(t)=\lim _{s \rightarrow t+} \theta_{k}^{\infty}(s)$ para $t \notin D_{k}$, sendo que esse limite é tomado sobre $s \in D_{k}$.

Para completar a prova, só temos que mostrar que $\theta_{k}^{\infty}$ é contínua à direita sobre $D_{k}$. Para um $t \in D_{k}$ fixado, tome $\theta_{k}^{\infty}(t+)=\lim _{s \rightarrow t+} \theta_{k}^{\infty}(s)$, esse limite existe q.c. por causa da monotonicidade.

Comecemos tratando o caso $k=1$. Tomemos $\mathcal{G}$ a $\sigma$-álgebra gerada pelo meio aleatório $\left\{\gamma_{k}(x \mid k)\right\}$. 
Para um $s>0$ e $\lambda>0$ arbitrários, usando a Proposição 3.5, podemos calcular:

$$
\begin{aligned}
\mathbb{E}\left[e^{-\lambda \tilde{\theta}_{1}^{n}(s)} \mid \mathcal{G}\right] & =\exp \left\{-s \sum_{x_{1}} h_{\left.x\right|_{1}}\left(\sum_{x_{2}} h_{\left.x\right|_{2}}\left(\cdots \sum_{x_{n}} h_{\left.x\right|_{n}}\left(\lambda W\left(\left.x\right|_{n}\right)\right) \cdots\right)\right)\right\} \\
& =: \exp \left\{-s \phi_{n}(\lambda)\right\}
\end{aligned}
$$

Sabemos que $\widetilde{\theta}_{1}^{n}(s)$ converge q.c. para $\theta_{1}^{\infty}(s)$ quando $n \rightarrow \infty$. Dessa forma $e^{-\lambda \widetilde{\theta}_{1}^{n}(s)}$ também converge q.c. para $e^{-\lambda \theta_{1}^{\infty}(s)}$. Como estas variáveis são limitadas então esta convergência também é $L_{1}$. Dessa forma suas esperanças condicionais também convergem em $L_{1}$, isso é:

$$
\mathbb{E}\left[\exp \left\{-\lambda \theta_{1}^{n}(s)\right\} \mid \mathcal{G}\right]=\exp \left\{-s \phi_{n}(\lambda)\right\} \underset{n \rightarrow \infty}{\stackrel{L_{1}}{\longrightarrow}} \mathbb{E}\left[\exp \left\{-\lambda \theta_{1}^{\infty}(s)\right\} \mid \mathcal{G}\right]
$$

De onde concluímos que existe uma função aleatória $\phi(\lambda)$ tal que $\mathbb{E}\left[e^{-s \phi_{n}(\lambda)}\right]$ converge para $\mathbb{E}\left[e^{-s \phi(\lambda)}\right]$ e $\mathbb{E}\left[e^{-\lambda \theta_{1}^{\infty}(s)} \mid \mathcal{G}\right]=e^{-s \phi(\lambda)}$.

Com isso podemos calcular a transformada de Laplace de $\theta_{1}^{\infty}(t+)-\theta_{1}^{\infty}(t)$ usando a Proposição 3.5 e o fato de que $\theta_{1}^{n}$ têm incrementos estacionários (Proposição 2.14):

$$
\begin{aligned}
\mathbb{E} & {\left[\exp \left\{-\lambda\left(\theta_{1}^{\infty}(t+)-\theta_{1}^{\infty}(t)\right)\right\}\right] } \\
& =\lim _{s \rightarrow 0+} \lim _{n \rightarrow \infty} \mathbb{E}\left[\exp \left\{-\lambda \widetilde{\theta}_{1}^{n}(s)\right\}\right] \\
& =\lim _{s \rightarrow 0+} \lim _{n \rightarrow \infty} \mathbb{E}\left[\mathbb{E}\left[\exp \left\{-\lambda \widetilde{\theta}_{1}^{n}(s)\right\} \mid \mathcal{G}\right]\right] \\
& =\lim _{s \rightarrow 0+} \mathbb{E}[\exp \{-s \phi(\lambda)\}]=1 .
\end{aligned}
$$

As trocas entre limites e valores esperados podem ser justificadas pelo Teorema da Convergência Dominada. Com isso provamos que $\theta_{1}^{\infty}(t+)=\theta_{1}^{\infty}(t)$ q.c. para todo $t \in D_{1}$. Isso conclui a prova para o caso $k=1$.

Para o caso $k>1$, note que nossa escolha de $D_{k}$ garante que, com probabilidade $1, X_{k-1}(t) \in$ $\mathbb{N}_{*}^{k-1}$ para todo $t \in D_{k}$.

Dessa forma $t$ pertence à um intervalo $[a, b)$ tal que $X_{k-1}(s)=\left.x\right|_{k-1}$ para todo $s \in[a, b)$. Assim $L_{k-1}\left(\left.y\right|_{k-1}, s\right)$ é constante nesse intervalo para todo $\left.y\right|_{k-1} \neq\left. x\right|_{k-1}$. Utilizando a Proposição 3.4 e o caso anterior, podemos concluir:

$$
\begin{aligned}
\theta_{k}^{\infty}(t+)-\theta_{k}^{\infty}(t) & =\lim _{s \rightarrow 0+} \lim _{n \rightarrow \infty} \theta_{k}^{n}(t+s)-\theta_{k}^{n}(t) \\
& =\lim _{s \rightarrow 0+} \lim _{n \rightarrow \infty} \sum_{\left.y\right|_{k-1}}\left[\theta_{\left.y\right|_{k-1}}^{n}\left(L_{k-1}\left(\left.y\right|_{k-1}, t+s\right)\right)-\theta_{\left.y\right|_{k-1}}^{n}\left(L_{k-1}\left(\left.y\right|_{k-1}, t\right)\right)\right] \\
& =\lim _{s \rightarrow 0+} \lim _{n \rightarrow \infty} \theta_{\left.x\right|_{k-1}}^{n}\left(L_{k-1}\left(\left.x\right|_{k-1}, t+s\right)\right)-\theta_{\left.x\right|_{k-1}}^{n}\left(L_{k-1}\left(\left.x\right|_{k-1}, t\right)\right) \\
& =\lim _{s \rightarrow 0+} \theta_{\left.x\right|_{k-1}}^{\infty}\left(L_{k-1}\left(\left.x\right|_{k-1}, t+s\right)\right)-\theta_{\left.x\right|_{k-1}}^{\infty}\left(L_{k-1}\left(\left.x\right|_{k-1}, t\right)\right)=0 .
\end{aligned}
$$

\subsection{Trivialidade dos Relógios Limite}

O Teorema 3.6 diz apenas que $\theta_{k}^{\infty}$ é não decrescente, não afirmando que esse processo é estritamente crescente. O Teorema 3.7 abaixo vai nos dizer que é possível que $\theta_{k}^{\infty}$ seja identicamente igual à zero com probabilidade 1, caso que chamaremos de trivial. Felizmente o Teorema 3.8 nos dará 
condições sobre as quais $\theta_{k}^{\infty}$ é estritamente crescente e cresce para o infinito. Chamaremos esse caso de não trivial.O objetivo dessa seção é provar esses dois teoremas.

Teorema 3.7 (Trivialidade). Suponha que:

$$
\sum_{k=1}^{\infty}\left(1-\alpha_{k}\right)<\infty, \quad \sum_{k=1}^{\infty} \frac{1-\alpha_{k+1}}{1-\alpha_{k}}=\infty,
$$

então $\theta_{k}^{\infty}(t)=0$ q.c. para todo $t \geqslant 0$ e $k \in \mathbb{N}_{*}$.

Teorema 3.8 (Não Trivialidade). Suponha que:

$$
\sum_{k=1}^{\infty} \frac{1-\alpha_{k+1}}{1-\alpha_{k}}<\infty
$$

então, para todo $k \in \mathbb{N}_{*}, \theta_{k}^{\infty}$ é q.c. uma função estritamente crescente e $\lim _{t \rightarrow \infty} \theta_{k}^{\infty}(t)=\infty$.

Observação 3.9. Note que o caso $\sum_{k}\left(1-\alpha_{k}\right)=\infty$ não é coberto pelo Teorema 3.7 ou pelo Teorema 3.8. Acreditamos, por cálculos realizados em exemplos e intuição adquirida ao trabalhar no problema, que os relógios limites também são triviais nesse caso. Assim preferimos não investigar mais a fundo esse caso pois ele parece tecnicamente difícil e esperamos obter apenas um resultado negativo ao persegui-lo.

Nós vamos nos referir à condição (3.13) como condição de não trivialidade. Note que ela implica que $\sum_{i}\left(1-\alpha_{i}\right)<\infty$.

Os dois teoremas serão provados estudando o comportamento do exponente de Laplace de $\tilde{\theta}_{k}^{n}$. Para tornar a notação dessa seção mais compacta, vamos definir, para $n \geqslant k$ e $\lambda \geqslant 0$ :

$$
Z_{\left.x\right|_{k}}^{n}:= \begin{cases}\lambda W\left(\left.x\right|_{k}\right) & \text { if } k=n, \\ \sum_{x_{k+1}} h_{\left.x\right|_{k+1}}\left(Z_{\left.x\right|_{k+1}}^{n}\right) & \text { caso contrário. }\end{cases}
$$

A função $h$ usada aqui é a mesma usada ao calcular a transformada de Laplace de $\tilde{\theta}_{k}^{n}$ em (3.10). Vamos começar provando um resultado auxiliar que será útil na demonstração dos dois teoremas.

Lema 3.10. Suponha que $\sum_{i}\left(1-\alpha_{i}\right)<\infty$, então $\sum_{i} \frac{1-\alpha_{i+1}}{1-\alpha_{i}}<\infty$ se e somente se $\sum_{i}\left(1-d_{i}\right)<\infty$, onde:

$$
d_{i}:=\frac{\alpha_{i} \Gamma\left(\alpha_{i}\right) \Gamma\left(1-\alpha_{i}\right)}{\Gamma\left(1-\alpha_{i} / \alpha_{i+1}\right)} .
$$

Demonstração. Expandindo a definição de $d_{i}$ e usando que $\Gamma(1+\alpha)=\alpha \Gamma(\alpha)$, podemos escrever:

$$
\begin{aligned}
\Gamma & \left(2-\frac{\alpha_{i}}{\alpha_{i+1}}\right) \frac{\left(1-d_{i}\right)\left(1-\alpha_{i}\right)}{1-\alpha_{i+1}} \\
& =\frac{1-\alpha_{i}}{1-\alpha_{i+1}}\left[\Gamma\left(2-\frac{\alpha_{i}}{\alpha_{i+1}}\right)-\Gamma\left(2-\alpha_{i}\right)\right] \\
& +\frac{\Gamma\left(2-\alpha_{i}\right)}{1-\alpha_{i+1}}\left[1-\alpha_{i}-\left(1-\frac{\alpha_{i}}{\alpha_{i+1}}\right)\right] \\
& +\frac{\Gamma\left(2-\alpha_{i}\right)}{1-\alpha_{i+1}}\left(1-\frac{\alpha_{i}}{\alpha_{i+1}}\right)\left[1-\Gamma\left(1+\alpha_{i}\right)\right]
\end{aligned}
$$


Note que os três termos são positivos para $i$ grande o suficiente, já que $\Gamma$ é decrescente perto de 1 e crescente perto de 2 e $0<\alpha_{i}<\alpha_{i} / \alpha_{i+1}<1$.

Como $\Gamma$ é diferenciável no intervalo [1,2], então (3.15) converge para zero quando $i \rightarrow \infty$. Um cálculo direto nos mostra que (3.16) converge para 1 quando $i \rightarrow \infty$.

Tome $a_{i}:=(3.15)+(3.16)$ e $b_{i}:=(3.17)$, podemos escrever:

$$
b_{i}^{\prime}:=b_{i} \frac{1-\alpha_{i+1}}{1-\alpha_{i}}=\frac{\alpha_{i+1}-\alpha_{i}}{\alpha_{i+1}} \frac{\Gamma(2)-\Gamma\left(1+\alpha_{i}\right)}{1-\alpha_{i}}=\frac{\Gamma(2)-\Gamma\left(1+\alpha_{i}\right)}{\alpha_{i}\left(1-\alpha_{i}\right)}\left[\left(1-\alpha_{i}\right)-\left(1-\alpha_{i+1}\right)\right] .
$$

Portanto $b_{i}^{\prime}$ é somável, já que $\sum_{i}\left(1-\alpha_{i}\right)<\infty$ e $\left(\Gamma(2)-\Gamma\left(1+\alpha_{i}\right)\right) /\left(1-\alpha_{i}\right)$ converge para uma constante quando $i \rightarrow \infty$.

Finalmente podemos escrever:

$$
\left(1-d_{i}\right) \Gamma\left(2-\frac{\alpha_{i}}{\alpha_{i+1}}\right)=a_{i} \frac{1-\alpha_{i+1}}{1-\alpha_{i}}+b_{i}^{\prime}
$$

Como $a_{i} \rightarrow 1$ e $\Gamma\left(2-\alpha_{i} / \alpha_{i+1}\right) \rightarrow 1$ quando $i \rightarrow \infty$ e $b_{i}^{\prime}$ é somável, então concluímos que $\sum_{i}\left(1-d_{i}\right)<\infty$ se e somente se $\sum_{i} \frac{1-\alpha_{i+1}}{1-\alpha_{i}}<\infty$.

Demonstração do Teorema 3.7. Vamos provar apenas que $\theta_{1}^{\infty} \equiv 0$. A extensão dessa prova para o caso geral é direta.

Com base na Proposição 3.5 e (3.14), é suficiente provar que $Z_{\varnothing}^{n}$ converge para 0 em probabilidade quando $n \rightarrow \infty$. Nós vamos mostrar que $\mathbb{E}\left(Z_{\varnothing}^{n}\right) \rightarrow 0$ quando $n \rightarrow \infty$, um resultado ligeiramente mais forte. Para isso definimos:

$$
a_{k}^{n}= \begin{cases}\mathbb{E}\left(Z_{\left.x\right|_{k}}^{n}\right) & \text { if } k<n, \\ \mathbb{E}\left(\left(Z_{\left.x\right|_{k}}^{n}\right)^{\alpha_{k}}\right) & \text { if } k=n .\end{cases}
$$

Usando a última afirmação da Proposição 3.1, junto com a Proposição A.3, podemos calcular $a_{n}^{n}=\lambda^{\alpha_{n}} \Gamma\left(1-\alpha_{n} / \alpha_{n+1}\right) / \Gamma\left(1-\alpha_{n}\right)$. A Proposição A.1 nos permite utilizar o Teorema de Campbell para obter que, quando $k<n$ :

$$
\begin{aligned}
a_{k-1}^{n} & =\mathbb{E}\left[\sum_{x_{k}} \frac{\gamma_{k}\left(\left.x\right|_{k}\right) Z_{\left.x\right|_{k}}^{n}}{1+\gamma_{k}\left(\left.x\right|_{k}\right) Z_{\left.x\right|_{k}}^{n}}\right] \\
& =\int_{0}^{\infty} \frac{x}{1+x} \frac{\mathbb{E}\left[\left(Z_{\left.x\right|_{k}}^{n}\right)^{\alpha_{k}}\right] c_{k}}{x^{1+\alpha_{k}}} d x .
\end{aligned}
$$

Agora podemos aplicar a desigualdade de Jensen, obtendo:

$$
\begin{aligned}
a_{k-1}^{n} & \leqslant c_{k}\left(a_{k}^{n}\right)^{\alpha_{k}} \int_{0}^{\infty} \frac{1}{x^{\alpha_{k}}(1+x)} d x \\
& =c_{k}\left(a_{k}^{n}\right)^{\alpha_{k}} \int_{0}^{1} y^{1-\alpha_{k}-1}(1-y)^{\alpha_{k}-1} d y \\
& =c_{k}\left(a_{k}^{n}\right)^{\alpha_{k}} \Gamma\left(1-\alpha_{k}\right) \Gamma\left(\alpha_{k}\right) \\
& =\left(a_{k}^{n}\right)^{\alpha_{k}} \frac{\alpha_{k} \Gamma\left(\alpha_{k}\right) \Gamma\left(1-\alpha_{k}\right)}{\Gamma\left(1-\frac{\alpha_{k}}{\alpha_{k+1}}\right)} .
\end{aligned}
$$


Podemos calcular $a_{n-1}^{n}$ de maneira análoga, mas usando o valor exato de $\mathbb{E}\left[\left(Z_{\left.x\right|_{n}}^{n}\right)^{\alpha_{n}}\right]$ em vez de uma estimativa obtida da desigualdade de Jensen, obtendo:

$$
a_{n-1}^{n}=a_{n}^{n} \frac{\alpha_{n} \Gamma\left(\alpha_{n}\right) \Gamma\left(1-\alpha_{n}\right)}{\Gamma\left(1-\frac{\alpha_{n}}{\alpha_{n+1}}\right)}=\lambda^{\alpha_{n}} \alpha_{n} \Gamma\left(\alpha_{n}\right) .
$$

Iterando sobre (3.18) e usando (3.19), obtemos:

$$
\begin{aligned}
a_{0}^{n} & \leqslant \prod_{i=1}^{n-1}\left[\frac{\alpha_{i} \Gamma\left(\alpha_{i}\right) \Gamma\left(1-\alpha_{i}\right)}{\Gamma\left(1-\frac{\alpha_{i}}{\alpha_{i+1}}\right)}\right]^{\alpha_{1} \ldots \alpha_{i-1}}\left(a_{n-1}^{n}\right)^{\alpha_{1} \ldots \alpha_{n-1}} \\
& =\left(\lambda^{\alpha_{n}} \alpha_{n} \Gamma\left(\alpha_{n}\right)\right)^{\alpha_{1} \ldots \alpha_{n-1}} \prod_{i=1}^{n-1}\left[\frac{\alpha_{i} \Gamma\left(\alpha_{i}\right) \Gamma\left(1-\alpha_{i}\right)}{\Gamma\left(1-\frac{\alpha_{i}}{\alpha_{i+1}}\right)}\right]^{\alpha_{1} \ldots \alpha_{i-1}} .
\end{aligned}
$$

Note que $\lambda^{\prod_{j} \alpha_{j}} \leqslant \max \{\lambda, 1\}$ e que $\alpha_{n} \Gamma\left(\alpha_{n}\right)=\Gamma\left(1+\alpha_{n}\right) \rightarrow 1$ quando $n \rightarrow \infty$. Então, se mostrarmos que o produto em (3.20) converge à zero quando $n \rightarrow \infty$, vai seguir que $a_{0}^{n} \stackrel{n \rightarrow \infty}{\longrightarrow} 0$. Isso motiva a definição:

$$
d_{i}:=\frac{\alpha_{i} \Gamma\left(\alpha_{i}\right) \Gamma\left(1-\alpha_{i}\right)}{\Gamma\left(1-\frac{\alpha_{i}}{\alpha_{i+1}}\right)}, \quad \quad b_{i}:=\left[d_{i}\right]^{\alpha_{1} \ldots \alpha_{i-1}} .
$$

Queremos mostrar que $\prod_{i=1}^{\infty} b_{i}=0$. Note que $d_{i}, b_{i} \in(0,1)$, já que $\Gamma$ é uma função decrescente em $(0,1)$ e $\alpha \Gamma(\alpha)=\Gamma(\alpha+1)<1$ para $\alpha \in(0,1)$.

Por hipótese, $\sum_{i}\left(1-\alpha_{i}\right)<\infty$. Isso implica que $\prod_{i} \alpha_{i}>0$. Portanto:

$$
\begin{aligned}
\prod_{i} b_{i}=0 & \Leftrightarrow \sum_{i} \log b_{i}=-\infty \Leftrightarrow \sum_{i} \alpha_{1} \ldots \alpha_{i-1} \log d_{i}=-\infty \\
& \Leftrightarrow \sum_{i} \log d_{i}=-\infty \Leftrightarrow \prod_{i} d_{i}=0 \Leftrightarrow \sum_{i}\left(1-d_{i}\right)=+\infty .
\end{aligned}
$$

Finalmente o Lema 3.10 garante que $\sum_{i}\left(1-d_{i}\right)=\infty$ quando $\sum_{i} \frac{1-\alpha_{i+1}}{1-\alpha_{i}}=\infty$.

Antes de provar o Teorema 3.8, vamos estabelecer um resultado auxiliar:

Lema 3.11. Tome $\left\{\gamma_{i}: i \in \mathbb{N}_{*}\right\}$ as marcas de um Processo Pontual de Poisson com medida intensidade $\mu(d x)=\frac{c}{x^{1+\alpha}} \mathbb{I}\{x>0\}$, para algum $c>0$ e $\alpha \in(0,1)$. Tome $Z:=\sum_{i} \frac{\gamma_{i}}{1+\gamma_{i}}$. Então para todo $\beta \in(0,1)$ :

$$
\mathbb{E}\left(Z^{\beta}\right) \geqslant \frac{c \Gamma(\alpha) \Gamma(1-\alpha)}{[1+c \Gamma(\alpha) \Gamma(1-\alpha)]^{1-\beta}} .
$$

Demonstração. Tome $\phi(\theta):=\mathbb{E}\left(e^{-\theta Z}\right)$ a transformada de Laplace de $Z$. Podemos calcular essa quantidade usando o Teorema de Campbell:

$$
\begin{aligned}
\phi(\theta) & =\exp \left\{-\int_{0}^{\infty}\left(1-e^{-\theta \frac{x}{x+1}}\right) \frac{c}{x^{1+\alpha}} d x\right\} \\
& =\exp \left\{-c \int_{0}^{1} \frac{1-e^{-\theta y}}{y^{1+\alpha}(1-y)^{1-\alpha}} d y\right\} \\
& =\exp \{-c \psi(\theta)\} .
\end{aligned}
$$


A primeira derivada de $\psi$ pode ser calculada e limitada por:

$$
\begin{aligned}
\psi^{\prime}(\theta) & =\lim _{h \rightarrow 0} \frac{\psi(\theta+h)-\psi(\theta)}{h} \\
& =\lim _{h \rightarrow 0} \int_{0}^{1} \frac{e^{-\theta y}}{y^{1+\alpha}(1-y)^{1-\alpha}} \frac{1-e^{-h y}}{h} d y \\
& =\int_{0}^{1} \frac{e^{-\theta y}}{y^{\alpha}(1-y)^{1-\alpha}} d y \\
& \geqslant \int_{0}^{1} \frac{e^{-\theta}}{y^{\alpha}(1-y)^{1-\alpha}} d y \\
& =e^{-\theta} \Gamma(\alpha) \Gamma(1-\alpha),
\end{aligned}
$$

justificamos a igualdade entre a segunda e terceira linha usando o Teorema de Convergência Dominada. Como $1-e^{-x} \leqslant x$, temos que:

$$
\begin{aligned}
\psi(\theta) & =\int_{0}^{1} \frac{1-e^{-\theta y}}{y^{1+\alpha}(1-y)^{1-\alpha}} d y \\
& \leqslant \theta \int_{0}^{1} \frac{1}{y^{\alpha}(1-y)^{1-\alpha}} d y \\
& =\theta \Gamma(\alpha) \Gamma(1-\alpha), \\
\phi(\theta) & =e^{-c \psi(\theta)} \\
& \geqslant e^{-c \theta \Gamma(\alpha) \Gamma(1-\alpha)}
\end{aligned}
$$

Finalmente podemos aplicar a Proposição A.2, concluindo:

$$
\begin{aligned}
\mathbb{E}\left(X^{\beta}\right) & =-\frac{1}{\Gamma(1-\beta)} \int_{0}^{\infty} \theta^{-\beta} \phi^{\prime}(\theta) d \theta \\
& =\frac{1}{\Gamma(1-\beta)} \int_{0}^{\infty} \theta^{-\beta} c \phi(\theta) \psi^{\prime}(\theta) d \theta \\
& \geqslant \frac{c}{\Gamma(1-\beta)} \int_{0}^{\infty} \theta^{-\beta} e^{-c \theta \Gamma(\alpha) \Gamma(1-\alpha)} e^{-\theta} \Gamma(\alpha) \Gamma(1-\alpha) d \theta \\
& =\frac{c \Gamma(\alpha) \Gamma(1-\alpha)}{[1+c \Gamma(\alpha) \Gamma(1-\alpha)]^{1-\beta}} .
\end{aligned}
$$

Demonstração do Teorema 3.8. Vamos provar as afirmações do teorema apenas para $\theta_{1}^{\infty}$. Podemos estender a prova para o caso geral usando o fato de que $\widetilde{\theta}_{1}^{n}=\widetilde{\theta}_{k+1}^{n} \circ \theta_{1}^{k}$.

Definindo $a_{k}^{n}:=\mathbb{E}\left[\left(Z_{\left.x\right|_{k}}^{n}\right)^{\alpha_{k}}\right]$, vamos mostrar que $\liminf _{n \rightarrow \infty} a_{1}^{n}>0$. Com as Proposições 3.5 e A.1, podemos escrever:

$$
\mathbb{E}\left[e^{-\lambda \widetilde{\theta}_{1}^{n}(t)}\right]=\mathbb{E}\left[\exp \left\{-t \sum_{x 1} \gamma_{1}\left(\left.x\right|_{1}\right) Z_{\left.x\right|_{1}}^{n}\right\}\right]=\mathbb{E}\left[\exp \left\{-t \sum_{x 1} \gamma_{1}\left(\left.x\right|_{1}\right)\left(a_{1}^{n}\right)^{1 / \alpha_{1}}\right\}\right] .
$$

Se mostrarmos que $\liminf _{n \rightarrow \infty} a_{1}^{n}>0$, vai seguir da expressão acima que $\lim _{t \rightarrow \infty} \theta_{1}^{\infty}(t)=\infty \mathrm{em}$ probabilidade. Já que $\theta_{1}^{\infty}$ é q.c. não decrescente, segue que $\lim _{t \rightarrow \infty} \theta_{1}^{\infty}(t)=\infty$ q.c. .

Para mostrar que $\liminf _{n \rightarrow \infty} a_{1}^{n}>0$, comecemos aplicando o Lema 3.11 e a Proposição A.1, 
obtendo que para $k \leqslant n$ :

$$
\begin{aligned}
a_{k-1}^{n} & \geqslant \frac{c_{k} a_{k}^{n} \Gamma\left(\alpha_{k}\right) \Gamma\left(1-\alpha_{k}\right)}{\left[1+c_{k} a_{k}^{n} \Gamma\left(\alpha_{k}\right) \Gamma\left(1-\alpha_{k}\right)\right]^{1-\alpha_{k-1}}} \\
& =\frac{a_{k}^{n} \frac{\alpha_{k} \Gamma\left(\alpha_{k}\right) \Gamma\left(1-\alpha_{k}\right)}{\Gamma\left(1-\alpha_{k} / \alpha_{k+1}\right)}}{\left[1+a_{k}^{n} \frac{\alpha_{k} \Gamma\left(\alpha_{k}\right) \Gamma\left(1-\alpha_{k}\right)}{\Gamma\left(1-\alpha_{k} / \alpha_{k+1}\right)}\right]^{1-\alpha_{k-1}}} .
\end{aligned}
$$

Para trabalhar com o denominador, observemos (3.20). Apesar da definição de $a_{k}^{n}$ ser ligeiramente diferente naquela prova, podemos utilizar a desigualdade de Jensen para obter:

$$
\begin{aligned}
a_{k}^{n} & :=\mathbb{E}\left[\left(Z_{\left.x\right|_{k}}^{n}\right)^{\alpha_{k}}\right] \leqslant\left(\mathbb{E}\left[Z_{\left.x\right|_{k}}^{n}\right]\right)^{\alpha_{k}} \\
& \leqslant\left(\left(\lambda^{\alpha_{n}} \alpha_{n} \Gamma\left(\alpha_{n}\right)\right)^{\alpha_{k+1} \ldots \alpha_{n-1}} \prod_{i=k+1}^{n-1}\left[\frac{\alpha_{i} \Gamma\left(\alpha_{i}\right) \Gamma\left(1-\alpha_{i}\right)}{\Gamma\left(1-\frac{\alpha_{i}}{\alpha_{i+1}}\right)}\right]^{\alpha_{k+1} \ldots \alpha_{i-1}}\right)^{\alpha_{k}} \\
& \leqslant \lambda^{\alpha_{k} \ldots \alpha_{n}} \leqslant \max \{\lambda, 1\} .
\end{aligned}
$$

Tomando $\delta:=\max \{\lambda, 1\}$ e usando essa última expressão no denominador de (3.22), obtemos:

$$
a_{k-1}^{n} \geqslant a_{k}^{n}(1+\delta)^{-\left(1-\alpha_{k-1}\right)} \frac{\alpha_{k} \Gamma\left(\alpha_{k}\right) \Gamma\left(1-\alpha_{k}\right)}{\Gamma\left(1-\alpha_{k} / \alpha_{k+1}\right)} .
$$

Já que $0<\alpha_{i}<\alpha_{i} / \alpha_{i+1}<1$ e $\Gamma$ é decrescente perto do zero, podemos iterar essa desigualdade para obter:

$$
\begin{aligned}
a_{k}^{n} & \geqslant \lambda^{\alpha_{n}} \frac{\Gamma\left(1-\frac{\alpha_{n}}{\alpha_{n+1}}\right)}{\Gamma\left(1-\alpha_{n}\right)}(1+\delta)^{-\sum_{j=k}^{n-1}\left(1-\alpha_{j}\right)} \prod_{j=k+1}^{n} \frac{\alpha_{j} \Gamma\left(\alpha_{j}\right) \Gamma\left(1-\alpha_{j}\right)}{\Gamma\left(1-\frac{\alpha_{j}}{\alpha_{j+1}}\right)} \\
& \geqslant \lambda^{\alpha_{n}}(1+\delta)^{-\sum_{j=k}^{n-1}\left(1-\alpha_{j}\right)} \prod_{j=k+1}^{n} \frac{\alpha_{j} \Gamma\left(\alpha_{j}\right) \Gamma\left(1-\alpha_{j}\right)}{\Gamma\left(1-\frac{\alpha_{j}}{\alpha_{j+1}}\right)} .
\end{aligned}
$$

Note que os termos do produto ao final da última expressão são exatamente iguais à $d_{i}$, definido no Lema 3.10. Mostramos naquele lema que, sempre que $\sum_{i} \frac{1-\alpha_{i+1}}{1-\alpha_{i}}<\infty$, vai valer que $\sum_{i}\left(1-d_{i}\right)<$ $\infty$, o que implica que $\prod_{i} d_{i}>0$.

Portanto concluímos que $\liminf _{n \rightarrow \infty} a_{k}^{n}>0$. Para completar a prova, precisamos mostrar que $\theta_{1}^{\infty}$ é q.c. estritamente crescente. Para isso vamos mostrar que $\lim _{\lambda \rightarrow \infty} \lim _{\inf _{n \rightarrow \infty}} Z_{\varnothing}^{n}(\lambda)=\infty$ em probabilidade.

Já que só estamos interessados em valores altos de $\lambda$, podemos supor que $\lambda>1$. Tomando uma constante $C>0$ tal que $\prod_{i=1}^{\infty} d_{i}>C$ e usando a definição de $\delta$, podemos reescrever (3.24):

$$
\begin{aligned}
a_{k}^{n}(\lambda) & \geqslant C \lambda^{\alpha_{n}}(1+\lambda)^{-\sum_{j=k}^{n-1}\left(1-\alpha_{j}\right)} \\
\liminf _{n \rightarrow \infty} a_{k}^{n}(\lambda) & \geqslant C \lambda(1+\lambda)^{-\sum_{j=k}^{\infty}\left(1-\alpha_{j}\right)} .
\end{aligned}
$$

Fixemos um $k$ tal que $\sum_{i \geqslant k}\left(1-\alpha_{i}\right)<1$. Da última expressão concluímos que para esse $k$ vale que $\lim _{\lambda \rightarrow \infty} \liminf _{n \rightarrow \infty} a_{k}^{n}(\lambda)=\infty$. 
Usando a Proposição A.1, e tomando um $M \in \mathbb{N}$ arbitrário, podemos escrever:

$$
\begin{aligned}
Z_{\left.x\right|_{k-1}}^{n} & =\sum_{x_{k}} \frac{\gamma_{k}\left(\left.x\right|_{k}\right) Z_{\left.x\right|_{k}}^{n}(\lambda)}{1+\gamma_{k}\left(\left.x\right|_{k}\right) Z_{\left.x\right|_{k}}^{n}(\lambda)} \\
& =\sum_{x_{k}} \frac{\gamma_{k}\left(\left.x\right|_{k}\right)\left(a_{k}^{n}(\lambda)\right)^{1 / \alpha_{k}}}{1+\gamma_{k}\left(\left.x\right|_{k}\right)\left(a_{k}^{n}(\lambda)\right)^{1 / \alpha_{k}}} \\
& \geqslant \sum_{x_{k}=1}^{M} \frac{\gamma_{k}\left(\left.x\right|_{k}\right)\left(a_{k}^{n}(\lambda)\right)^{1 / \alpha_{k}}}{1+\gamma_{k}\left(\left.x\right|_{k}\right)\left(a_{k}^{n}(\lambda)\right)^{1 / \alpha_{k}}} \underset{n \rightarrow \infty, \lambda \rightarrow \infty}{\longrightarrow} M .
\end{aligned}
$$

Já que $M$ pode ser tomando arbitrariamente grande, concluímos que $\lim _{\lambda \rightarrow \infty} \liminf _{n \rightarrow \infty} Z_{\left.x\right|_{k-1}}^{n}(\lambda)=$ $\infty$ em probabilidade. Sabendo que $Z_{\left.x\right|_{k-2}}^{n}=\sum_{x_{k-1}} h_{\left.x\right|_{k-1}}\left(Z_{\left.x\right|_{k-1}}^{n}\right)$, podemos usar argumentos análogos para mostrar que $\lim _{\lambda \rightarrow \infty} \liminf _{n \rightarrow \infty} Z_{\left.x\right|_{k-2}}^{n}=\infty$ em probabilidade. Iterando concluímos que:

$\lim _{\lambda \rightarrow \infty} \liminf _{n \rightarrow \infty} Z_{\varnothing}^{n}(\lambda)=\infty$ em probabilidade. 


\section{Capítulo 4}

\section{O Processo K em uma Árvore de Profundidade Infinita}

Nesse capítulo vamos construir, sob certas condições, o Processo K em uma árvore de profundidade infinita, e provar que ele é o limite dos Processos $\mathrm{K}$ em árvores de profundidade finita, estudados no Capítulo 2, quando a profundidade das árvores cresce para o infinito.

\subsection{Definição do Processo}

A Proposição 2.8 nos sugere uma maneira de definir o Processo K em uma árvore de profundidade infinita, através dos relógios limites construídos e definidos no Capítulo 3.

Definição 4.1. O Processo K numa árvore com profundidade infinita é um processo estocástico em tempo contínuo $\mathbb{Y}=\left(Y_{k}\right)_{k \in \mathbb{N}_{*}}$, tomando valores em $\overline{\mathbb{N}}_{*}^{\mathbb{N} *}$, onde:

$$
Y_{k}(t)= \begin{cases}x & \text { se } t \in \bigcup_{i=1}^{\infty}\left[\theta_{k}^{\infty}\left(\sigma_{i}^{k, x}-\right), \theta_{k}^{\infty}\left(\sigma_{i}^{k, x}\right)\right), \\ \infty \quad \text { caso contrário. }\end{cases}
$$

Note que essa construção faz sentido mesmo no caso trivial, isso é, quando $\theta_{k}^{\infty}(t)=0$ q.c. para todo $t \geqslant 0$. Porém nesse caso $Y_{k}(t)=\infty$ para todo $t \geqslant 0$.

Após construir esse processo $\mathbb{Y}$, é natural perguntar se ele seria o limite dos processos $X_{k}$ definidos no Capítulo 2. Vamos responder esse pergunta de maneira afirmativa com o Teorema 4.15 na Seção 4.4 .

\subsection{Convergência dos Relógios}

Antes de podermos responder perguntas sobre a convergência dos Processos K, precisamos responder perguntas sobre a convergência dos relógios. O resultado principal dessa seção é o Teorema 4.5 , que estabelece que $\theta_{k}^{n}$ converge para $\theta_{k}^{\infty}$ quando $n \rightarrow \infty$ em probabilidade de maneira uniforme em compactos.

Lema 4.1. Suponha que $\frac{1-\alpha_{k+1}}{1-\alpha_{k}} \rightarrow 0$ quando $k \rightarrow \infty$, o que é verdade sob a condição de não trivialidade (3.13), então:

$$
\lim _{k \rightarrow \infty} \mathbb{E}\left[\left|W\left(\left.x\right|_{k}\right)-1\right|^{\alpha_{k}}\right]=0
$$


Demonstração. A Proposição 1.1.12 de Samorodnitsky e Taqqu [ST94] diz como obter a função característica de uma variável aleatória estável positiva a partir de sua transformada de Laplace. Como a Proposição 3.1 nos fornece a transformada de Laplace de $W\left(\left.x\right|_{k}\right)$ então podemos obter a função característica de $W\left(\left.x\right|_{k}\right)-1$ :

$$
\begin{aligned}
\varphi_{k}(u) & :=\mathbb{E}\left[e^{i u\left(W\left(\left.x\right|_{k}\right)-1\right)}\right] \\
& =\exp \left\{-|u|^{\alpha_{k+1}}\left[\cos \left(\frac{\pi \alpha_{k+1}}{2}\right)-i \operatorname{sgn}(u) \sin \left(\frac{\pi \alpha_{k+1}}{2}\right)\right]-i u\right\} \\
& =\exp \left\{-|u|^{\alpha_{k+1}} \cos \left(\frac{\pi \alpha_{k+1}}{2}\right)-i\left[u-|u|^{\alpha_{k+1}} \operatorname{sgn}(u) \sin \left(\frac{\pi \alpha_{k+1}}{2}\right)\right]\right\} .
\end{aligned}
$$

O Teorema 2.2 de Laue [Lau80] afirma que:

$$
\mathbb{E}\left[\left|W\left(\left.x\right|_{k}\right)-1\right|^{\alpha_{k}}\right]=\frac{1}{\cos \left(\frac{\pi \alpha_{k}}{2}\right)} \operatorname{Re}\left[\frac{\alpha_{k}}{\Gamma\left(1-\alpha_{k}\right)} \int_{0}^{\infty} \frac{1-\varphi_{k}(-u)}{u^{1+\alpha_{k}}} d u\right] .
$$

Note que $\frac{\alpha_{k}}{\cos \left(\frac{\pi \alpha_{k}}{2}\right) \Gamma\left(1-\alpha_{k}\right)}$ converge para $\frac{2}{\pi}$ quando $k \rightarrow \infty$. Assim resta provar que a parte real da integral converge para zero. Fixemos um $\epsilon>0$ arbitrário e podemos escrever:

$$
\begin{aligned}
\operatorname{Re} & {\left[\int_{0}^{\infty} \frac{1-\varphi_{k}(-u)}{u^{1+\alpha_{k}}} d u\right] } \\
& =\int_{0}^{\infty} \frac{1}{u^{1+\alpha_{k}}}\left[1-\exp \left\{-u^{\alpha_{k+1}} \cos \left(\frac{\pi \alpha_{k+1}}{2}\right)\right\} \cos \left(u-u^{\alpha_{k+1}} \sin \left(\frac{\pi \alpha_{k+1}}{2}\right)\right)\right] d u \\
& =\int_{0}^{\epsilon} \frac{1}{u^{1+\alpha_{k}}}\left[1-\exp \left\{-u^{\alpha_{k+1}} \cos \left(\frac{\pi \alpha_{k+1}}{2}\right)\right\} \cos \left(u-u^{\alpha_{k+1}} \sin \left(\frac{\pi \alpha_{k+1}}{2}\right)\right)\right] d u \\
& +\int_{\epsilon}^{\infty} \frac{1}{u^{1+\alpha_{k}}}\left[1-\exp \left\{-u^{\alpha_{k+1}} \cos \left(\frac{\pi \alpha_{k+1}}{2}\right)\right\} \cos \left(u-u^{\alpha_{k+1}} \sin \left(\frac{\pi \alpha_{k+1}}{2}\right)\right)\right] d u .
\end{aligned}
$$

Para controlar (4.5), note que a função sendo integrada converge à zero quando $k \rightarrow \infty \mathrm{e}$ pode ser limitada por $2 / u^{1+\alpha_{k}} \leqslant 2 / u^{3 / 2}$ para $k$ grande o suficiente. Portanto, pelo o Teorema da Convergência Dominada, (4.5) converge para zero quando $k \rightarrow \infty$ para qualquer escolha de $\epsilon>0$.

Para controlar (4.4), podemos usar que $1-e^{-x} \leqslant x$ :

$$
\begin{aligned}
(4.4) & \leqslant \int_{0}^{\epsilon} \frac{1}{u^{1+\alpha_{k}}}\left[u^{\alpha_{k+1}} \cos \left(\frac{\pi \alpha_{k+1}}{2}\right)-\log \cos \left(u-u^{\alpha_{k+1}} \sin \left(\frac{\pi \alpha_{k+1}}{2}\right)\right)\right] d u \\
& =\int_{0}^{\epsilon} \frac{1}{u^{1+\alpha_{k}}} u^{\alpha_{k+1}} \cos \left(\frac{\pi \alpha_{k+1}}{2}\right) d u-\int_{0}^{\epsilon} \frac{1}{u^{1+\alpha_{k}}} \log \cos \left(u-u^{\alpha_{k+1}} \sin \left(\frac{\pi \alpha_{k+1}}{2}\right)\right) d u \\
& =\frac{\epsilon^{\alpha_{k+1}-\alpha_{k}}}{\alpha_{k+1}-\alpha_{k}} \cos \left(\frac{\pi \alpha_{k+1}}{2}\right)-\int_{0}^{\epsilon} \frac{1}{u^{1+\alpha_{k}}} \log \cos \left(u-u^{\alpha_{k+1}} \sin \left(\frac{\pi \alpha_{k+1}}{2}\right)\right) d u .
\end{aligned}
$$

Por hipótese vale que $\frac{1-\alpha_{k+1}}{1-\alpha_{k}} \stackrel{k \rightarrow \infty}{\longrightarrow} 0$ e é verdade que $\cos (\pi x / 2) /(1-x) \stackrel{x \rightarrow 1}{\longrightarrow} \frac{\pi}{2}$, com isso podemos reescrever o termo mais a esquerda em (4.6):

$$
\begin{array}{r}
\frac{\epsilon^{\alpha_{k+1}-\alpha_{k}}}{\alpha_{k+1}-\alpha_{k}} \cos \left(\frac{\pi \alpha_{k+1}}{2}\right)=\epsilon^{\alpha_{k+1}-\alpha_{k}} \frac{1-\alpha_{k+1}}{\alpha_{k+1}-\alpha_{k}} \frac{\cos \left(\frac{\pi \alpha_{k+1}}{2}\right)}{1-\alpha_{k+1}} \\
=\epsilon^{\alpha_{k+1}-\alpha_{k}}\left(\frac{1-\alpha_{k}}{1-\alpha_{k+1}}-1\right)^{-1} \frac{\cos \left(\frac{\pi \alpha_{k+1}}{2}\right)}{1-\alpha_{k+1}} \stackrel{k \rightarrow \infty}{\longrightarrow} 0 .
\end{array}
$$

Para controlar a integral em (4.6), vamos usar que existe um $\epsilon_{0}>0$ tal que se $|x|<\epsilon_{0}$ então 
$-\log \cos x<x^{2}$. Isso pode ser provado calculando as duas primeiras derivadas dessa quantidade e usando que cosseno é uma função par. Dessa forma, para $\epsilon>0$ pequeno o suficiente:

$$
\begin{aligned}
-\int_{0}^{\epsilon} & \frac{1}{u^{1+\alpha_{k}}} \log \cos \left(u-u^{\alpha_{k+1}} \sin \left(\frac{\pi \alpha_{k+1}}{2}\right)\right) d u \\
& \leqslant \int_{0}^{\epsilon} \frac{1}{u^{1+\alpha_{k}}}\left(u-u^{\alpha_{k+1}} \sin \left(\frac{\pi \alpha_{k+1}}{2}\right)\right)^{2} d u \\
& =\int_{0}^{\epsilon} u^{1-\alpha_{k}}-2 u^{\alpha_{k+1}-\alpha_{k}} \sin \left(\frac{\pi \alpha_{k+1}}{2}\right)+u^{2 \alpha_{k+1}-\alpha_{k}-1} \sin ^{2}\left(\frac{\pi \alpha_{k+1}}{2}\right) d u \\
& =\frac{\epsilon^{2-\alpha_{k}}}{2-\alpha_{k}}-2 \frac{\epsilon^{\alpha_{k+1}-\alpha_{k}+1}}{\alpha_{k+1}-\alpha_{k}+1} \sin \left(\frac{\pi \alpha_{k+1}}{2}\right)+\frac{\epsilon^{2 \alpha_{k+1}-\alpha_{k}}}{2 \alpha_{k+1}-\alpha_{k}} \sin ^{2}\left(\frac{\pi \alpha_{k+1}}{2}\right) \\
& \stackrel{k \rightarrow \infty}{\longrightarrow} 0 .
\end{aligned}
$$

Proposição 4.2 (Convergência finito dimensional). Se $\sum_{k}\left(1-\alpha_{k}\right)<\infty$, então para todo $t>0$ fixado e $k \in \mathbb{N}_{*}, \theta_{k}^{n}(t)$ converge em probabilidade para $\theta_{k}^{\infty}(t)$ quando $n \rightarrow \infty$.

Demonstração. Vamos supor que $\frac{1-\alpha_{k+1}}{1-\alpha_{k}} \rightarrow 0$ quando $k \rightarrow \infty$. Quando isso não for verdade, então as condições do Teorema 3.7 serão válidas, e podemos utilizar um argumento análogo ao da prova daquele teorema para mostrar que $\theta_{k}^{n}(t) \stackrel{n \rightarrow \infty}{\longrightarrow} 0$ em probabilidade para todo $t>0$.

Iremos provar que $\mathbb{E}\left[e^{-\left|\theta_{k}^{n}(t)-\widetilde{\theta}_{k}^{n}(t)\right|}\right]$ converge para 1 quando $n \rightarrow \infty$, o que implica que $\theta_{k}^{n}(t)$ converge em probabilidade para $\theta_{k}^{\infty}(t)$.

Vamos começar considerando o caso $k=1$. Tomando $Z_{\left.x\right|_{n}}=\left|W\left(\left.x\right|_{n}\right)-1\right|$ na prova da Proposição 3.5, obtemos:

$$
\begin{aligned}
\mathbb{E}\left[\exp \left\{-\left|\theta_{1}^{n}(t)-\widetilde{\theta}_{1}^{n}(t)\right|\right\}\right] & \geqslant \mathbb{E}\left[\exp \left\{-\sum_{\left.x\right|_{n}} L\left(\left.x\right|_{n}, \theta_{1}^{n}(t)\right)\left|1-W\left(\left.x\right|_{n}\right)\right|\right\}\right] \\
& =\mathbb{E}\left[\exp \left\{-t \sum_{x_{1}} h_{\left.x\right|_{1}}\left(\sum_{x_{2}} h_{\left.x\right|_{2}}\left(\cdots \sum_{x_{n}} h_{\left.x\right|_{n}}\left(\left|W\left(\left.x\right|_{n}\right)-1\right|\right) \cdots\right)\right)\right\}\right] .
\end{aligned}
$$

Prosseguindo como no Teorema 3.7, definimos:

$$
Z_{\left.x\right|_{k}}^{n}:=\left\{\begin{array}{ll}
\sum_{x_{k+1}} h_{\left.x\right|_{k+1}}\left(Z_{\left.x\right|_{k+1}}^{n}\right) & \text { se } k<n \\
\left|W\left(\left.x\right|_{n}\right)-1\right| & \text { se } k=n
\end{array} \quad a_{k}^{n}:= \begin{cases}\mathbb{E}\left[Z_{\left.x\right|_{k}}^{n}\right] & \text { se } k<n \\
\mathbb{E}\left[\left|W\left(\left.x\right|_{n}\right)-1\right|^{\alpha_{n}}\right] & \text { se } k=n .\end{cases}\right.
$$

Lema 4.1 garante que $a_{n}^{n} \rightarrow 0$ quando $n \rightarrow \infty$. Seguindo a prova do Teorema 3.7, obtemos (3.18) e a primeira igualdade de (3.19), de onde obtemos que $a_{k-1}^{n} \leqslant a_{k}^{n}$ para todo $k \leqslant n$. Portanto $a_{k}^{n} \leqslant a_{n}^{n} \stackrel{n \rightarrow \infty}{\longrightarrow} 0$ para todo $k<n$ e $Z_{\varnothing}^{n} \rightarrow 0$ na norma $L_{1}$ quando $n \rightarrow \infty$. Aplicando o Teorema da Convergência Dominada em (4.7), concluímos o caso $k=1$. 
Para o caso geral, usando a Proposição 3.4 e o resultado anterior, podemos escrever:

$$
\begin{aligned}
\mathbb{E}\left[e^{-\left|\theta_{k+1}^{n}(t)-\widetilde{\theta}_{k+1}^{n}(t)\right|}\right] & \geqslant \mathbb{E}\left[\exp \left\{-\sum_{\left.x\right|_{k}}\left|\theta_{\left.x\right|_{k}}^{n}\left(L\left(\left.x\right|_{k}, t\right)\right)-\widetilde{\theta}_{\left.x\right|_{k}}^{n}\left(L\left(\left.x\right|_{k}, t\right)\right)\right|\right\}\right] \\
& =\mathbb{E}\left[\exp \left\{-\sum_{\left.x\right|_{k}} L\left(\left.x\right|_{k}, t\right) Z_{\left.x\right|_{k}}^{n}\right\}\right] .
\end{aligned}
$$

Como $L\left(\left.x\right|_{k}, t\right)$ é independente de $Z_{\left.x\right|_{k}}^{n}$, então:

$$
\mathbb{E}\left[\sum_{\left.x\right|_{k}} L\left(\left.x\right|_{k}, t\right) Z_{\left.x\right|_{k}}^{n}\right]=\sum_{\left.x\right|_{k}} \mathbb{E}\left[L\left(\left.x\right|_{k}, t\right) Z_{\left.x\right|_{k}}^{n}\right]=t a_{k}^{n} \stackrel{n \rightarrow \infty}{\longrightarrow} 0
$$

Concluímos a prova aplicando o Teorema da Convergência Dominada em (4.8).

Lema 4.3. Quase certamente:

$$
\sup _{n} \sum_{\left.x\right|_{n}} \bar{\gamma}_{n}\left(\left.x\right|_{n}\right)<\infty
$$

Demonstração. Tome $A_{n}:=\left\{\left.x\right|_{n} \in \mathbb{N}_{*}^{n}: \bar{\gamma}_{n}\left(\left.x\right|_{n}\right)>1\right\}$ e $m_{n}:=\max \left\{\bar{\gamma}_{n}\left(\left.x\right|_{n}\right):\left.x\right|_{n} \in \mathbb{N}_{*}^{n}\right\}$. Usando a Proposição 3.1, sabemos que quase certamente:

$$
\begin{aligned}
& W(\varnothing)=\lim _{n \rightarrow \infty} \sum_{\left.x\right|_{n} \in \mathbb{N}_{*}^{n}}\left(\bar{\gamma}_{n}\left(\left.x\right|_{n}\right)\right)^{\alpha_{n+1}} \geqslant \limsup _{n \rightarrow \infty} \sum_{\left.x\right|_{n} \in A_{n}}\left(\bar{\gamma}_{n}\left(\left.x\right|_{n}\right)\right)^{\alpha_{n+1}} \geqslant \limsup _{n \rightarrow \infty}\left|A_{n}\right|, \\
& W(\varnothing)=\lim _{n \rightarrow \infty} \sum_{\left.x\right|_{n} \in \mathbb{N}_{*}^{n}}\left(\bar{\gamma}_{n}\left(\left.x\right|_{n}\right)\right)^{\alpha_{n+1}} \geqslant \limsup _{n \rightarrow \infty}\left(m_{n}\right)^{\alpha_{n+1}}=\limsup _{n \rightarrow \infty} m_{n} .
\end{aligned}
$$

Como $W(\varnothing)<\infty$, então $\lim \sup _{n}\left|A_{n}\right|<\infty$ e $\lim \sup _{n} m_{n}<\infty$ q.c. . Portanto:

$$
\begin{aligned}
\sum_{\left.x\right|_{n}} \bar{\gamma}_{n}\left(\left.x\right|_{n}\right) & =\sum_{\left.x\right|_{n} \in A_{n}} \bar{\gamma}_{n}\left(\left.x\right|_{n}\right)+\sum_{\left.x\right|_{n} \notin A_{n}} \bar{\gamma}_{n}\left(\left.x\right|_{n}\right) \\
& \leqslant m_{n}\left|A_{n}\right|+\sum_{\left.x\right|_{n} \notin A_{n}}\left(\bar{\gamma}_{n}\left(\left.x\right|_{n}\right)\right)^{\alpha_{n+1}} .
\end{aligned}
$$

Por causa do último comentário, a primeira parcela dessa soma é limitada q.c. por uma constante, enquanto que o limsup da segunda é dominado por $W(\varnothing)$, o que termina a prova.

Lema 4.4. Se $\left\{\gamma_{k}\left(\left.x\right|_{k}\right): k \in \mathbb{N}_{*},\left.x\right|_{k} \in \mathbb{N}_{*}^{k}\right\}$ são fixados e $\sum_{i}\left(1-\alpha_{i}\right)<\infty$ então $\theta_{1}^{\infty}$ também é um subordinador

Demonstração. Ao definir $\theta_{1}^{\infty}$ no Teorema 3.6, nós já mostramos que esse processo é não decrescente e contínuo a direita. Resta apenas mostrar que ele têm incrementos estacionários e independentes.

Da proposição 4.2 , sabemos que $\theta_{1}^{n}$ converge pontualmente em probabilidade para $\theta_{1}^{\infty}$ quando $n \rightarrow \infty$. Dessa forma, se tomarmos $0=t_{0}<t_{1}<\ldots<t_{m}<\infty$ e $A_{1}, \ldots, A_{m}$ borelianos tais que $\mathbb{P}\left(\theta_{1}^{\infty}\left(t_{i}\right)-\theta_{1}^{\infty}\left(t_{i-1}\right) \in \partial A_{i}\right)=0$. Então podemos usar o fato que $\theta_{1}^{n}$ são subordinadores, provado na 
Proposição 2.14, para calcular:

$$
\begin{aligned}
\mathbb{P}\left(\bigcap_{i=1}^{m}\left\{\theta_{1}^{\infty}\left(t_{i}\right)-\theta_{1}^{\infty}\left(t_{i-1}\right) \in A_{i}\right\}\right) & =\lim _{n \rightarrow \infty} \mathbb{P}\left(\bigcap_{i=1}^{m}\left\{\theta_{1}^{n}\left(t_{i}\right)-\theta_{1}^{n}\left(t_{i-1}\right) \in A_{i}\right\}\right) \\
& =\lim _{n \rightarrow \infty} \prod_{i=1}^{m} \mathbb{P}\left(\theta_{1}^{n}\left(t_{i}\right)-\theta_{1}^{n}\left(t_{i-1}\right) \in A_{i}\right) \\
& =\lim _{n \rightarrow \infty} \prod_{i=1}^{m} \mathbb{P}\left(\theta_{1}^{n}\left(t_{i}-t_{i-1}\right) \in A_{i}\right) \\
& =\prod_{i=1}^{m} \lim _{n \rightarrow \infty} \mathbb{P}\left(\theta_{1}^{n}\left(t_{i}-t_{i-1}\right) \in A_{i}\right) \\
& =\prod_{i=1}^{m} \mathbb{P}\left(\theta_{1}^{\infty}\left(t_{i}-t_{i-1}\right) \in A_{i}\right) .
\end{aligned}
$$

Teorema 4.5. Suponha que $\sum_{k}\left(1-\alpha_{k}\right)<\infty$, então, para cada $k \in \mathbb{N}^{*}, \theta_{k}^{n}-\theta_{k}^{\infty}$ converge em distribuição para a função identicamente nula na topologia de Skorohod quando $n \rightarrow \infty$.

Observação 4.6. Esse é um resultado mais forte que simplesmente dizer que $\theta_{k}^{n}$ converge em distribuição para $\theta_{k}^{\infty}$ na topologia de Skorohod. Convergência na topologia de Skorohod para uma função contínua é equivalente à convergência uniforme em compactos.

Demonstração do Teorema 4.5. Sob a condição de trivialidade (3.12), este teorema é um corolário direto da Proposição 4.2, junto com a observação de que cada $\theta_{k}^{n}$ é uma função monótona não decrescente. Dessa maneira vamos supor a condição de não trivialidade (3.13).

Comecemos com o caso $k=1$. Fixemos o meio aleatório $\gamma=\left\{\gamma_{k}\left(\left.x\right|_{k}\right): k \geqslant 1,\left.x\right|_{k} \in \mathbb{N}_{*}^{k}\right\}$ e mostremos a convergência para quase todas essas escolhas.

Já que mostramos a convergência finito dimensional na Proposição 4.2, então o Teorema 7.8 do Capítulo 3 de [EK86] afirma que se $\left\{\left|\theta_{1}^{n}-\theta_{1}^{\infty}\right|\right\}$ é relativamente compacto, então teremos a convergência em distribuição para a função identicamente nula.

A parte (b) do Teorema 8.6 do Capítulo 3 de [EK86] nos afirma que para mostrar compacidade relativa é suficiente mostrar que, para $0<s<\delta$ e $t>0$, quase certamente:

$$
\mathbb{E}_{\gamma}\left[\left|\left(\theta_{1}^{n}(t+s)-\theta_{1}^{\infty}(t+s)\right)-\left(\theta_{1}^{n}(t)-\theta_{1}^{\infty}(t)\right)\right| \mid \mathcal{F}_{t}^{n}\right] \leqslant 2 \delta \sup _{m \in \mathbb{N}} \sum_{\left.x\right|_{m}} \bar{\gamma}_{m}\left(\left.x\right|_{m}\right) \underset{a . s .}{\stackrel{\delta \rightarrow 0}{\longrightarrow}} 0
$$

onde $\mathcal{F}_{t}^{n}$ é a $\sigma$-álgebra gerada por $\left\{\theta_{1}^{n}(r)-\theta_{1}^{\infty}(r): r \leqslant t\right\}$.

A convergência quase certa é uma consequência direta do Lema 4.3. Para provar a desigualdade, tome $\mathcal{F}_{t}$ a $\sigma$-álgebra gerada pelas variáveis aleatórias $\left\{\theta_{1}^{n}(r): r \leqslant t, n \in \mathbb{N}_{*}\right\}$. Como $\mathcal{F}_{t}^{n} \subseteq \mathcal{F}_{t}$, podemos escrever:

$$
\begin{aligned}
\mathbb{E}_{\gamma} & {\left[\left|\left(\theta_{1}^{n}(t+s)-\theta_{1}^{\infty}(t+s)\right)-\left(\theta_{1}^{n}(t)-\theta_{1}^{\infty}(t)\right)\right| \mid \mathcal{F}_{t}^{n}\right] } \\
& \leqslant \mathbb{E}_{\gamma}\left[\theta_{1}^{n}(t+s)-\theta_{1}^{n}(t) \mid \mathcal{F}_{t}^{n}\right]+\mathbb{E}_{\gamma}\left[\theta_{1}^{\infty}(t+s)-\theta_{1}^{\infty}(t) \mid \mathcal{F}_{t}^{n}\right] \\
& \leqslant \mathbb{E}_{\gamma}\left[\mathbb{E}_{\gamma}\left[\theta_{1}^{n}(t+s)-\theta_{1}^{n}(t) \mid \mathcal{F}_{t}\right] \mid \mathcal{F}_{t}^{n}\right]+\mathbb{E}_{\gamma}\left[\mathbb{E}_{\gamma}\left[\theta_{1}^{\infty}(t+s)-\theta_{1}^{\infty}(t) \mid \mathcal{F}_{t}\right] \mid \mathcal{F}_{t}^{n}\right]
\end{aligned}
$$

Note que $\theta_{1}^{n}(t+s)-\theta_{1}^{n}(t)$ só depende dos valores de $\theta_{1}^{m}(r)$ para $m \geqslant n$ e $r \leqslant t$ através dos valores de $\theta_{1}^{n}(r), r \leqslant t$. Então podemos usar a Proposição 2.14 para calcular o valor da primeira 
parcela:

$$
\mathbb{E}_{\gamma}\left[\theta_{1}^{n}(t+s)-\theta_{1}^{n}(t) \mid \mathcal{F}_{t}\right]=\mathbb{E}_{\gamma}\left[\theta_{1}^{n}(s)\right]=s \sum_{\left.x\right|_{n}} \bar{\gamma}_{n}\left(\left.x\right|_{n}\right)
$$

Já que $\theta_{1}^{n}(t)$ e $\theta_{1}^{n}(t+s)$ convergem em probabilidade para $\theta_{1}^{\infty}(t)$ e $\theta_{1}^{\infty}(t+s)$ respectivamente (Proposição 4.2), então podemos tomar uma sequência crescente $\left(n_{m}\right)_{m}$ tal que essa convergência seja quase certa sobre essa sequência. Usando o Lema de Fatou, podemos concluir:

$$
\mathbb{E}_{\gamma}\left[\theta_{1}^{\infty}(t+s)-\theta_{1}^{\infty}(t) \mid \mathcal{F}_{t}\right] \leqslant \liminf _{m \rightarrow \infty} \mathbb{E}_{\gamma}\left[\theta_{1}^{n_{m}}(t+s)-\theta_{1}^{n_{m}}(t) \mid \mathcal{F}_{t}\right] \leqslant \liminf _{m \rightarrow \infty} s \sum_{\left.x\right|_{n_{m}}} \bar{\gamma}_{n_{m}}\left(\left.x\right|_{n_{m}}\right)
$$

Aplicando os dois últimos resultados em (4.11) nos fornece (4.10) e completa a prova da convergência de $\theta_{1}^{n}-\theta_{1}^{\infty}$.

Para o caso geral, note que $\theta_{k+1}^{n}-\theta_{k+1}^{\infty}$ converge em probabilidade para a função identicamente nula na topologia de Skorohod se e somente se para todo $T>0$ :

$$
\sup _{0 \leqslant t \leqslant T}\left|\theta_{k+1}^{n}(t)-\theta_{k+1}^{\infty}(t)\right| \underset{n \rightarrow \infty}{\stackrel{P}{\longrightarrow}} 0
$$

Para cada $\left.x\right|_{k} \in \mathbb{N}_{*}^{k}$ fixado, construa $\theta_{\left.x\right|_{k}}^{\infty}$ a partir de $\theta_{k+1}^{\infty}$ de maneira análoga à feita na Definição 3.2. Usando a Proposição 3.4 e o último caso, concluímos que $\theta_{\left.x\right|_{k}}^{n}-\theta_{\left.x\right|_{k}}^{\infty}$ converge para uma função identicamente nula em probabilidade na topologia de Skorohod quando $n \rightarrow \infty$.

Tome $V_{1} \subset V_{2} \subset \ldots \mathbb{N}_{*}^{k}$ uma sequência de conjuntos de $N_{*}^{k}$ tais que $\bigcup_{l=1}^{\infty} V_{l}=\mathbb{N}_{*}^{k}$ e cada $V_{l}$ é finito. Para um $\epsilon>0$ arbitrário, fixe um $l$ tal que $\mathbb{P}\left(\sum_{\left.x\right|_{k} \notin V_{l}} \theta_{\left.x\right|_{k}}^{\infty}\left(L\left(\left.x\right|_{k}, T\right)\right)>\epsilon\right)<\epsilon$. Com essa escolha:

$$
\begin{aligned}
& \sup _{0 \leqslant t \leqslant T}\left|\theta_{k+1}^{n}(t)-\theta_{k+1}^{\infty}(t)\right|=\sup _{0 \leqslant t \leqslant T}\left|\sum_{\left.x\right|_{k}} \theta_{\left.x\right|_{k}}^{n}\left(L\left(\left.x\right|_{k}, t\right)\right)-\theta_{\left.x\right|_{k}}^{\infty}\left(L\left(\left.x\right|_{k}, t\right)\right)\right| \\
& \quad \leqslant \sup _{0 \leqslant t \leqslant T} \sum_{\left.x\right|_{k} \in V_{l}}\left|\theta_{\left.x\right|_{k}}^{n}\left(L\left(\left.x\right|_{k}, t\right)\right)-\theta_{\left.x\right|_{k}}^{\infty}\left(L\left(\left.x\right|_{k}, t\right)\right)\right|+\sum_{\left.x\right|_{k} \notin V_{l}} \theta_{\left.x\right|_{k}}^{n}\left(L\left(\left.x\right|_{k}, T\right)\right)+\sum_{\left.x\right|_{k} \notin V_{l}} \theta_{\left.x\right|_{k}}^{\infty}\left(L\left(\left.x\right|_{k}, T\right)\right) \\
& \quad \leqslant \sup _{0 \leqslant t \leqslant T} \sum_{\left.x\right|_{k} \in V_{l}}\left|\theta_{\left.x\right|_{k}}^{n}(t)-\theta_{\left.x\right|_{k}}^{\infty}(t)\right|+\sum_{\left.x\right|_{k} \notin V_{l}} \theta_{\left.x\right|_{k}}^{n}\left(L\left(\left.x\right|_{k}, T\right)\right)+\sum_{\left.x\right|_{k} \notin V_{l}} \theta_{\left.x\right|_{k}}^{\infty}\left(L\left(\left.x\right|_{k}, T\right)\right) .
\end{aligned}
$$

A primeira parcela converge para 0 em probabilidade quando $n \rightarrow \infty$ porque $V_{l}$ é finito. $\mathrm{O}$ terceiro termo é controlado pela nossa esolha de $l$. Usando um argumento análogo ao usado em (4.8), podemos mostrar que o segundo termo converge para o terceiro em probabilidade quando $n \rightarrow \infty$.

Observação 4.7. Utilizando de argumentos análogos, podemos provar o Teorema 4.5 com $\tilde{\theta}_{k}^{n}$ no lugar de $\theta_{k}^{n}$. A única mudança significativa na prova vem da igualdade $\mathbb{E}_{\gamma}\left[\widetilde{\theta}_{1}^{n}(t)\right]=t W(\varnothing)$ em (4.10). No entanto essa mudança acaba simplificando a demonstração. 


\subsection{Propriedades do Processo K em uma árvore de profundidade infinita}

A convergência dos relógios finitos para os relógios infinitos obtida no Teorema 4.5 nos dá uma ferramenta poderosa para analisar o comportamentos dos relógios infinitos. Nessa seção obteremos uma melhor caracterização destes relógios, conseguindo novas ferramentas que serão úteis ao tratar do Processo K em árvores de profundidade infinita.

Corolário 4.8. Para cada $j, k \in \mathbb{N}_{*}, j<k$, se $\sum_{i}\left(1-\alpha_{i}\right)<\infty$, vale que:

$$
\theta_{j}^{\infty}=\theta_{k}^{\infty} \circ \theta_{j}^{k-1} \quad \text { q.c.. }
$$

Demonstração. O Teorema 4.5 nos diz que $\theta_{j}^{n}$ e $\theta_{k}^{n}$ convergem em probabilidade uniformemente em compactos para $\theta_{j}^{\infty}$ e $\theta_{k}^{\infty}$ respectivamente. Tome uma sequência crescente $\left(n_{m}\right)_{m}$ de modo que tenhamos convergência seja quase certa sobre essa sequência.

Lembremos que, por construção $\theta_{j}^{n}=\theta_{k}^{n} \circ \theta_{j}^{k-1}$. Dessa forma, para todo $T>0$ :

$$
\begin{aligned}
\sup _{t \in[0, T]}\left|\theta_{j}^{\infty}(t)-\theta_{k}^{\infty}\left(\theta_{j}^{k-1}(t)\right)\right| & \leqslant \sup _{t \in[0, T]}\left|\theta_{j}^{\infty}(t)-\theta_{j}^{n_{m}}(t)\right|+\sup _{t \in[0, T]}\left|\theta_{k}^{n_{m}}\left(\theta_{j}^{k-1}(t)\right)-\theta_{k}^{\infty}\left(\theta_{j}^{k-1}(t)\right)\right| \\
& \underset{a \rightarrow \infty .}{\stackrel{m \rightarrow \infty}{\longrightarrow}} 0 .
\end{aligned}
$$

Corolário 4.9. Suponha que $\sum_{i}\left(1-\alpha_{i}\right)<\infty$. Para qualquer $k \in \mathbb{N}_{*}$, quase certamente, se $s \geqslant 0$ é um ponto de descontinuidade de $\theta_{k}^{\infty}$, então $s \in\left\{\sigma_{i}^{k, x}: i, x \in \mathbb{N}_{*}\right\}$.

Demonstração. Com o Teorema 4.5, podemos tomar uma sequência crescente $\left(n_{m}\right)_{m}$ tal que, para qualquer $T>s$ fixado:

$$
\sup _{t \in[0, T]}\left|\theta_{k}^{n_{m}}(t)-\theta_{k}^{\infty}(t)\right| \underset{q . c .}{\stackrel{m \rightarrow \infty}{\longrightarrow}} 0 .
$$

Já que $s$ é um ponto de descontinuidade de $\theta_{k}^{\infty}$ e essa é q.c. uma função não decrescente e contínua a direita, então existe um $\epsilon>0$ tal que $\theta_{k}^{\infty}(s)-\theta_{k}^{\infty}(s-)>\epsilon$, o que implica que $\theta_{k}^{\infty}(s)-\theta_{k}^{\infty}(s-h)>\epsilon$ para todo $h \in(0, s)$.

Para um $h \in(0, s)$ arbitrário:

$$
\begin{aligned}
\theta_{k}^{n_{m}}(s)-\theta_{k}^{n_{m}}(s-h) & =\theta_{k}^{n_{m}}(s)-\theta_{k}^{\infty}(s) \\
& +\theta_{k}^{\infty}(s)-\theta_{k}^{\infty}(s-h) \\
& +\theta_{k}^{\infty}(s-h)-\theta_{k}^{n_{m}}(s-h) .
\end{aligned}
$$

A primeira e terceira parcelas dessa última equação convergem para zero q.c. , uniformemente em $h$, quando $m \rightarrow \infty$. No entanto o segundo termo é sempre maior que $\epsilon$. De onde concluímos que $\inf _{h \in(0, s)} \theta_{k}^{n_{m}}(s)-\theta_{k}^{n_{m}}(s-h)>\epsilon / 2$ para $m$ grande o suficiente, então $s$ é um ponto de descontinuidade de $\theta_{k}^{n_{m}}$.

Finalmente podemos aplicar a Proposição 2.9 que afirma que $s \in\left\{\sigma_{i}^{k, x}: i, x \in \mathbb{N}_{*}\right\}$.

Corolário 4.10. Sob a suposição de não trivialidade (3.13), quase certamente, para cada $k \in \mathbb{N}_{*}$ :

$$
\theta_{k}^{\infty}\left(\mathbb{R}^{+}\right)=\left\{t \geqslant 0: Y_{k}(t)=\infty\right\}
$$


Demonstração. Tome $t \in \theta_{k}^{\infty}\left(\mathbb{R}^{+}\right)$e um $s \geqslant 0$ tal que $\theta_{k}^{\infty}(s)=t$. Vamos supor por absurdo que $Y_{k}(t)=x<\infty$.

Por definição, já que $Y_{k}(t)=x$, existe $i \in \mathbb{N}_{*}$ tal que $\theta_{k}^{\infty}\left(\sigma_{i}^{k, x}-\right) \leqslant t<\theta_{k}^{\infty}\left(\sigma_{i}^{k, x}\right)$.

Como $t=\theta_{k}^{\infty}(s)$ e $\theta_{k}^{\infty}$ é estritamente crescente (Teorema 3.8), então a desigualdade da direita implica que $s<\sigma_{i}^{k, x}$, o que por sua vez implica que $t=\theta_{k}^{\infty}(s)<\theta_{k}^{\infty}\left(\sigma_{i}^{k, x}-\right)$, contradizendo a primeira desigualdade do último parágrafo. Portanto $Y_{k}(t)=\infty$.

Agora vamos tomar $t>0$ qual que $Y_{k}(t)=\infty$. Tome $s=\inf \left\{r>0: \theta_{k}^{\infty}(r)>t\right\}$. Pela continuidade à direita de $\theta_{k}^{\infty}$, concluímos que $\theta_{k}^{\infty}(s) \geqslant t$. Vamos supor, por absurdo, que $\theta_{k}^{\infty}(s)>t$. Pela definição de $s$ sabemos que $\theta_{k}^{\infty}(s-) \leqslant t$. Portanto $\theta_{k}^{\infty}(s-) \neq \theta_{k}^{\infty}(s)$.

Usando Corolário 4.9, temos que $s=\sigma_{i}^{k, x}$ para algum $i, x \in \mathbb{N}_{*}$. Dessa maneira $\theta_{k}^{\infty}\left(\sigma_{i}^{k, x}-\right) \leqslant$ $t<\theta_{k}^{\infty}\left(\sigma_{i}^{k, x}\right)$, o que implica que $Y_{k}(t)=x<\infty$, contradizendo nossa escolha para $t$.

Corolário 4.11. Se $\sum_{i}\left(1-\alpha_{i}\right)<\infty$, então:

$$
\left\{t \geqslant 0: Y_{k}(t)=\infty\right\} \subseteq\left\{t \geqslant 0: Y_{k+1}(t)=\infty\right\},
$$

quase certamente para todo $k \in \mathbb{N}_{*}$.

Demonstração. O Corolário 4.8 nos diz que, quase certamente:

$$
\theta_{k}^{\infty}\left(\mathbb{R}^{+}\right)=\left\{\theta_{k}^{\infty}(s): s \geqslant 0\right\}=\left\{\theta_{k+1}^{\infty}\left(\Xi_{k}(s)\right): s \geqslant 0\right\} \subseteq\left\{\theta_{k+1}^{\infty}(s): s \geqslant 0\right\}=\theta_{k+1}\left(\mathbb{R}^{+}\right)
$$

Aplicando o Corolário 4.10 concluímos:

$$
\left\{t \geqslant 0: Y_{k}(t)=\infty\right\}=\theta_{k}^{\infty}\left(\mathbb{R}^{+}\right) \subseteq \theta_{k+1}^{\infty}\left(\mathbb{R}^{+}\right)=\left\{t \geqslant 0: Y_{k+1}(t)=\infty\right\}
$$

Corolário 4.12. Sob a condição de não trivialidade (3.13), o conjunto $\left\{t \geqslant 0: Y_{k}(t)=\infty\right\}$ têm medida de Lebesgue nula quase certamente para cada $k \in \mathbb{N}_{*}$.

Demonstração. Vamos mostrar que a seguinte igualdade é válida quase certamente para todo $t>0$ :

$$
\theta_{k}^{\infty}(t)=\sum_{x=1}^{\infty} \sum_{i=1}^{N^{k, x}(t)} \theta_{k}^{\infty}\left(\sigma_{i}^{k, x}\right)-\theta_{k}^{\infty}\left(\sigma_{i}^{k, x}-\right) .
$$

Com isso estamos mostrando que $\theta_{k}^{\infty}$ é uma função escada, dessa forma sua imagem tem medida de Lebesgue nula. O resultado segue do Corolário 4.10.

Note que (4.13) não é uma consequência direta da convergência uniforme do Teorema 4.5. É possível construir uma sequência de funções escada $\left(f_{n}\right)$ que convergem uniformemente para outra função $f$, em que todas tenham exatamente as mesmas descontinuidades mas $f$ não é uma função escada.

Para o resto da prova vamos fixar o meio aleatório $\left\{\gamma_{k}\left(\left.x\right|_{k}\right)\right\}$ e provar o resultado para quase todas as escolhas desse meio.

Já que $\theta_{k}^{\infty}$ é não decrescente e contínua a direita, então ela é a função de distribuição acumulada de uma medida. A expressão no lado direito de (4.13) pode ser interpretada como a soma sobre 
alguns pontos dessa medida. Como medidas são $\sigma$-aditivas podemos concluir que:

$$
\theta_{k}^{\infty}(t) \geqslant \sum_{x=1}^{\infty} \sum_{i=1}^{N^{k, x}(t)} \theta_{k}^{\infty}\left(\sigma_{i}^{k, x}\right)-\theta_{k}^{\infty}\left(\sigma_{i}^{k, x}-\right) .
$$

Para mostrar a desigualdade inversa, usando a Observação 4.7, tome uma sequência crescente $\left(n_{m}\right)_{m}$ tal que sobre ela $\widetilde{\theta}_{k}^{n_{m}}$ convirja quase certamente uniformemente em compactos para $\theta_{k}^{\infty}$. Com essa escolha, para qualquer $N \in \mathbb{N}_{*}$ fixado:

$$
\begin{aligned}
\theta_{k}^{\infty}(t) & =\lim _{m \rightarrow \infty} \widetilde{\theta}_{k}^{n_{m}}(t) \\
& =\lim _{m \rightarrow \infty} \sum_{x=1}^{\infty} \sum_{i=1}^{N^{k, x}(t)} \widetilde{\theta}_{k}^{n_{m}}\left(\sigma_{i}^{k, x}\right)-\widetilde{\theta}_{k}^{n_{m}}\left(\sigma_{i}^{k, x}-\right) \\
& \leqslant \limsup _{m \rightarrow \infty} \sum_{x=1}^{N} \sum_{i=1}^{N^{k, x}(t)} \widetilde{\theta}_{k}^{n_{m}}\left(\sigma_{i}^{k, x}\right)-\widetilde{\theta}_{k}^{n_{m}}\left(\sigma_{i}^{k, x}-\right) \\
& +\limsup _{m \rightarrow \infty} \sum_{x=N+1}^{\infty} \sum_{i=1}^{N^{k, x}(t)} \widetilde{\theta}_{k}^{n_{m}}\left(\sigma_{i}^{k, x}\right)-\widetilde{\theta}_{k}^{n_{m}}\left(\sigma_{i}^{k, x}-\right) .
\end{aligned}
$$

Note que (4.14) é igual à:

$$
\sum_{x=1}^{N} \sum_{i=1}^{N^{k, x}(t)} \theta_{k}^{\infty}\left(\sigma_{i}^{k, x}\right)-\theta_{k}^{\infty}\left(\sigma_{i}^{k, x}-\right) \underset{q . c .}{\stackrel{N \rightarrow \infty}{\longrightarrow}} \sum_{x=1}^{\infty} \sum_{i=1}^{N^{k, x}(t)} \theta_{k}^{\infty}\left(\sigma_{i}^{k, x}\right)-\theta_{k}^{\infty}\left(\sigma_{i}^{k, x}-\right) .
$$

Para completar a prova, precisamos mostrar que (4.15) converge em probabilidade para zero quando $N \rightarrow \infty$. Com argumentos análogos aos usados no Teorema 3.6 podemos provar que $\left(\widetilde{\theta}_{k}^{n}\left(\sigma_{i}^{k, x}\right)-\widetilde{\theta}_{k}^{n}\left(\sigma_{i}^{k, x}-\right)\right)_{n}$ é um martingal. Dessa forma a sequência sobre a qual estamos tomando o limsup em (4.15) também é um martingal. Usando um Teorema de Convergência de Martingais [Dur10, Teorema 5.2.9], concluímos que o limsup naquela expressão é de fato um limite.

Denotando (4.15) por $K_{N}$ e tomando $\mathcal{F}_{k}$ a $\sigma$-álgebra formada por toda a informação até o nível 
$k$ podemos usar o Lema de Fatou para obter:

$$
\begin{aligned}
\mathbb{E}_{\gamma}\left[K_{N}\right] & \leqslant \liminf _{m \rightarrow \infty} \mathbb{E}_{\gamma}\left[\sum_{x=N+1}^{\infty} \sum_{i=1}^{N^{k, x}(t)} \widetilde{\theta}_{k}^{n_{m}}\left(\sigma_{i}^{k, x}\right)-\widetilde{\theta}_{k}^{n_{m}}\left(\sigma_{i}^{k, x}-\right)\right] \\
& =\liminf _{m \rightarrow \infty} \sum_{x=N+1}^{\infty} \mathbb{E}_{\gamma}\left[\sum_{i=1}^{N^{k, x}(t)} \mathbb{E}_{\gamma}\left[\widetilde{\theta}_{k}^{n_{m}}\left(\sigma_{i}^{k, x}\right)-\widetilde{\theta}_{k}^{n_{m}}\left(\sigma_{i}^{k, x}-\right) \mid \mathcal{F}_{k-1}\right]\right] \\
& =\liminf _{m \rightarrow \infty} \sum_{x=N+1}^{\infty} \mathbb{E}_{\gamma}\left[\sum_{i=1}^{N^{k, x}(t)} \gamma_{k}\left(X_{k-1}\left(\sigma_{i}^{k, x}\right) x\right) W\left(X_{k-1}\left(\sigma_{i}^{k, x}\right) x\right)\right] \\
& =\sum_{x=N+1}^{\infty} \mathbb{E}_{\gamma}\left[\sum_{i=1}^{N^{k, x}(t)} \gamma_{k}\left(X_{k-1}\left(\sigma_{i}^{k, x}\right) x\right) W\left(X_{k-1}\left(\sigma_{i}^{k, x}\right) x\right)\right] \\
& \leqslant \mathbb{E}_{\gamma}\left[\sum_{x=1}^{\infty} \sum_{i=1}^{N^{k, x}(t)} \gamma_{k}\left(X_{k-1}\left(\sigma_{i}^{k, x}\right) x\right) W\left(X_{k-1}\left(\sigma_{i}^{k, x}\right) x\right)\right] \\
& =\mathbb{E}_{\gamma}\left[\widetilde{\Xi}_{k}(t)\right]<\infty .
\end{aligned}
$$

Com isso obtemos que a soma em (4.16) é convergente q.c. e portanto converge para zero q.c. quando $N \rightarrow \infty$. Dessa forma $K_{N}$ converge para zero na métrica $L_{1}$ para quase todo $\gamma$ e dessa forma também converge em probabilidade.

\subsection{Convergência do Processo K}

Nosso objetivo nessa seção é provar um resultado de convergência dos Processos $\mathrm{K}$ em árvores de profundidade finita $\left(X_{k}\right)$ para o Processo $\mathrm{K}$ em uma árvore de profundidade infinita $(\mathbb{Y})$ quando profundidade dessas árvores cresce.

Para fazer isso, precisamos definir uma topologia no espaço de estados. Para uma coordenada fixa vamos usar uma compactificação de $\overline{\mathbb{N}}_{*}$. Especificamente, munimos $\overline{\mathbb{N}}_{*}$ com a métrica $\rho_{0}$ :

$$
\rho_{0}(x, y):=\left|\frac{1}{x}-\frac{1}{y}\right|, \quad x, y \in \overline{\mathbb{N}}_{*},
$$

sob a convenção de que $\frac{1}{\infty}=0$. Para $\overline{\mathbb{N}}_{*}^{\mathbb{N} *}$, adotamos a métrica $\rho$ :

$$
\rho\left(\left.x\right|_{\infty},\left.y\right|_{\infty}\right):=\sum_{k=1}^{\infty} \frac{\rho_{0}\left(x_{k}, y_{k}\right)}{2^{k}} .
$$

Dois pontos, $\left.x\right|_{\infty}$ e $\left.y\right|_{\infty}$, estão próximos nessa métrica se eles estão próximos num número finito de coordenadas. O que acontece em coordenadas "grandes" influencia pouco. Essa é uma métrica natural, visto que gera a topologia produto deste espaço.

Queremos estender a métrica $\rho$ para $\bigcup_{k=1}^{\infty} \overline{\mathbb{N}}_{*}^{k}$. Faremos isso adicionando um novo símbolo $\zeta$ para cada coordenada e definindo $\rho_{0}(\zeta, x):=\mathbb{\{}\{x=\zeta\}$. Depois estendemos $\rho$ ao "inserir $\zeta$ ao final" 
de $\left.x\right|_{k}$. Isso é, para $j \leqslant k \leqslant \infty$ :

$$
\begin{aligned}
& \rho\left(\left.x\right|_{j},\left.y\right|_{k}\right):=\sum_{i=1}^{j} \frac{\rho_{0}\left(x_{i}, y_{i}\right)}{2^{i}}+\sum_{i=j+1}^{k} \frac{\rho_{0}\left(\zeta, y_{i}\right)}{2^{i}}, \\
& \rho\left(\left.y\right|_{k},\left.x\right|_{j}\right):=\rho\left(\left.x\right|_{j},\left.y\right|_{k}\right) .
\end{aligned}
$$

Proposição 4.13. $\rho$ assim definida é uma métrica completa sobrecm $\overline{\mathbb{N}}_{*}^{\mathbb{N} *} \cup \bigcup_{k=1}^{\infty} \overline{\mathbb{N}}_{*}^{k}$ e gera uma topologia separável.

Demonstração. Omitiremos a prova de que $\rho$ é uma métrica.

Tomemos $D:=\bigcup_{k=1}^{\infty} \overline{\mathbb{N}}_{*}^{k}$, um conjunto enumerável. Para mostrar a separabilidade vamos mostrar que $D$ é denso.

Tome $y$ um ponto do espaço e $\epsilon>0$ arbitrários. Vamos mostrar que $D \cap B(y, \epsilon) \neq \varnothing$, onde $B(y, \epsilon)=\{x: \rho(x, y)<\epsilon\}$ é a bola aberta, centrada em $y$ de raio $\epsilon$.

Se $y \in D$ a afirmação é evidente, então vamos assumir que $y \in \overline{\mathbb{N}}_{*}^{\mathbb{N}_{*}}$. Fixe $n$ tal que $\sum_{i=n+1}^{\infty} \frac{1}{2^{i}}<\epsilon$, tome $x=\left(y_{1}, y_{2}, \ldots, y_{n}\right) \in D$ e note que:

$$
\rho(x, y)=\sum_{i=0}^{n} \frac{\rho_{0}\left(x_{i}, y_{i}\right)}{2^{i}}+\sum_{i>n} \frac{\rho_{0}\left(\zeta, y_{i}\right)}{2^{i}}=\sum_{i>n} \frac{1}{2^{i}}<\epsilon .
$$

Dessa forma $x \in D \cap B(y, \epsilon)$ e portanto essa intersecção não pode ser vazia.

Para mostrar que a métrica é completa, tome $x^{1}, x^{2}, \ldots$ uma sequência $\rho$-Cauchy de pontos do espaço, queremos mostrar que ela é convergente. Vamos cometer um abuso de notação e denotar os pontos do espaço como $x \in\left(\overline{\mathbb{N}}_{*} \cup\{\zeta\}\right)^{\mathbb{N} *}$.

Fixe uma coordenada $k$, vamos mostrar primeiro que existe um $x_{k} \in \overline{\mathbb{N}}_{*} \cup\{\zeta\}$ tal que $\lim _{n \rightarrow \infty} \rho_{0}\left(x_{k}, x_{k}^{n}\right)=$ 0. Para isso quebremos em dois casos:

1. Caso $\lim _{n \rightarrow \infty} \rho_{0}\left(x_{k}^{n}, \infty\right)=0$. Nesse caso tomamos $x_{k}=\infty$ e temos a propriedade desejada.

2. Caso contrário existe um $N \in \mathbb{N}_{*}$ e uma sequência crescente $\left(n_{m}\right)_{m}$ tal que $x_{k}^{n_{m}} \leqslant N$ para todo $m$. Aqui convencionamos que $\zeta<=N$ para todo $N \in \mathbb{N}_{*}$.

Afirmamos que existe um $m_{0}$ tal que $x_{k}^{n_{m}}$ é constante para $m>m_{0}$. Para provar isso, por absurdo, vamos supor que para todo $m_{0}$ existem $m_{1}, m_{2}>m_{0}$ tais que $x_{k}^{n_{m_{1}}} \neq x_{k}^{n_{m_{2}}}$. Fixando um $\epsilon<\left(\frac{1}{N}-\frac{1}{N-1}\right) \frac{1}{2^{k}}$ teremos que:

$$
\rho\left(x^{n_{m_{1}}}, x^{n_{m_{2}}}\right) \geqslant \frac{\rho_{0}\left(x_{k}^{n_{m_{1}}}, x_{k}^{n_{m_{2}}}\right)}{2^{k}} \geqslant\left(\frac{1}{N}-\frac{1}{N-1}\right) \frac{1}{2^{k}}>\epsilon .
$$

Isso contraria o fato que a sequência $x^{1}, x^{2}, \ldots$ é $\rho$-Cauchy. Vamos definir dessa forma $x_{k}$ como sendo esse valor constante que a sequência $x_{k}^{n_{m}}$ eventualmente assume. Afirmamos que $\lim _{n \rightarrow \infty} \rho_{0}\left(x_{k}, x_{k}^{n}\right)=0$.

Se isso não for verdade, então existe um $\epsilon>0$ e uma subsequência crescente $\left(n_{m}^{\prime}\right)_{m}$ tal que $\rho_{0}\left(x_{k}, x_{k}^{n_{m}^{\prime}}\right)>\epsilon$. Mas se isso for verdade, então para todo $n_{0}$ podemos tomar $n_{1}, n_{2}>n_{0}$, onde $n_{1}$ é um elemento da primeira sequência tal que $x_{k}^{n_{1}}=x_{k}$ e $n_{2}$ é da segunda sequência. Dessa forma:

$$
\rho\left(x^{n_{1}}, x^{n_{2}}\right) \geqslant \frac{\rho_{0}\left(x_{k}^{n_{1}}, x_{k}^{n_{2}}\right)}{2^{k}}>\frac{\epsilon}{2^{k}} .
$$


Novamente isso contraria o fato de $x^{1}, x^{2}, \ldots$ ser uma sequência $\rho$-Cauchy, o que completa a prova desse caso.

Agora tomemos $x=\left(x_{1}, x_{2}, \ldots\right)$ como dados por esse argumento. Note que se $x_{k}=\zeta$ para algum $k$, isso implica que $x_{k}^{n}=\zeta$ para $n$ suficientemente grande. Para tais valores de $n$ vai valer que $x_{j}^{n}=\zeta$ para $j>k$ e portanto $x_{j}=\zeta$. Assim $x$ é um ponto válido do espaço.

Fixado um $\epsilon>0$, sabemos que existe um $n_{k}$ tal que $\rho_{0}\left(x_{k}^{n}, x_{k}\right)<\epsilon / 2$ para todo $n>n_{k}$. Tomemos $k_{0}$ tal que $\sum_{i>k_{0}} \frac{1}{2^{i}}<\epsilon / 2$ e $n_{0}=\max \left(n_{1}, \ldots, n_{k_{0}}\right)$. Com essas escolhas, sabemos que para $n>n_{0}$ :

$$
\begin{array}{r}
\rho\left(x^{n}, x\right)=\sum_{i=0}^{k_{0}} \frac{\rho_{0}\left(x_{k}^{n}, x_{k}\right)}{2^{i}}+\sum_{i>k_{0}} \frac{\rho_{0}\left(x_{k}^{n}, x_{k}\right)}{2^{i}} \\
=\frac{\epsilon}{2} \sum_{i=0}^{k_{0}} \frac{1}{2^{i}}+\sum_{i>k_{0}} \frac{1}{2^{i}}<\epsilon .
\end{array}
$$

Dessa forma provamos que $x^{n}$ converge para $x$ quando $n \rightarrow \infty$.

Proposição 4.14. O Processo $K$ em uma árvore de profundidade infinita, $\mathbb{Y}$, quando $\sum_{i}\left(1-\alpha_{i}\right)<$ $\infty$, é quase certamente um processo càdlàg em relação à métrica $\rho$.

Demonstração. No caso trivial, isso é, quando (3.12) é válido, então $Y_{k}$ é constante e igual à $\infty$ para todo $k$, dessa forma esse processo é trivialmente càdlàg. Dessa forma vamos assumir a condição de não trivialidade (3.13) para o resto da demonstração.

Fixemos uma realização do processo, vamos mostrar primeiro que cada coordenada de $\mathbb{Y}$ representa uma função càdlàg com relação à métrica $\rho_{0}$. Isso é equivalente a mostrar que para todo $T>0$ e $\epsilon>0$, existem $0=t_{0}<t_{1} \ldots<t_{r}=T$ tais que $w_{k}\left[t_{i-1}, t_{i}\right)<\epsilon$, onde $w_{k}(A)=$ $\sup _{s, t \in A} \rho_{0}\left(Y_{k}(s), Y_{k}(t)\right)$.

Fixe um $M \in \mathbb{N}_{*}$ tal que $\frac{1}{M+1}<\epsilon$ e tome $t_{0}, t_{1}, \ldots, t_{r}$ uma ordenação do conjunto:

$$
\left(\left\{\theta_{k}^{\infty}\left(\sigma_{i}^{k, x}-\right), \theta_{k}^{\infty}\left(\sigma_{i}^{k, x}\right): i \in \mathbb{N}_{*}, x \leqslant M\right\} \cap[0, T]\right) \cup\{0, T\}
$$

Note que isso é possível pois esse conjunto é finito. Para um par de pontos $t_{i-1}, t_{i}$ consecutivos desse conjunto temos duas possibilidades:

- Caso $t_{i-1}=\theta_{k}^{\infty}\left(\sigma_{i}^{k, x}-\right)$ para algum $i, x \in \mathbb{N}_{*}$. Como $\theta_{k}^{\infty}$ é estritamente crescente, temos que $t_{i}=\theta_{k}^{\infty}\left(\sigma_{i}^{k, x}\right)$ ou $t_{i}=T<\theta_{k}^{\infty}\left(\sigma_{i}^{k, x}\right)$.

Nos dois casos $Y_{k}(t)=x$ para todo $t \in\left[t_{i-1}, t_{i}\right)$. Dessa forma $w_{k}\left[t_{i-1}, t_{i}\right)=0$.

- Caso o primeiro caso não seja verdade, então $Y_{k}(t)>M$ para todo $t \in\left[t_{i-1}, t_{i}\right)$. Dessa forma:

$$
w_{k}\left[t_{i-1}, t_{i}\right) \leqslant \sup _{x, y \in \mathbb{\mathbb { N }}_{*}} \rho_{0}(x, y)=\frac{1}{M+1}<\epsilon
$$

Portanto concluímos que cada $Y_{k}$ é uma função càdlàg. Agora queremos provar que $\mathbb{Y}$ também é. Para isso, analogamente ao que fizemos anteriormente, vamos mostrar que para todo $T>0$ e $\epsilon>0$ fixados arbitrariamente existem $0=t_{0}<t_{1}<t_{2}<\ldots<t_{r}=T$ tais que $w\left[t_{i-1}, t_{i}\right)<\epsilon$ para todo $i=1,2, \ldots, r$, onde $w(A)=\sup _{s, t \in A} \rho(\mathbb{Y}(t), \mathbb{Y}(s))$. 
Fixe $N \in \mathbb{N}_{*}$ tal que $\sum_{i>N} \frac{1}{2^{i}}=\frac{1}{2^{N}}<\frac{\epsilon}{2}$. E para cada $k \leqslant N$ tome $0=t_{0}^{k}<t_{1}^{k}<\ldots<t_{r_{k}}^{k}=T$ tais que $w_{k}\left[t_{i-1}^{k}, t_{i}^{k}\right)<\frac{\epsilon}{2}$. Finalmente defina $0=t_{0}<t_{1}<\ldots<t_{r}=T$ uma ordenação de todos os $t_{i}^{k}$ para $k \leqslant N, i \leqslant r_{k}$.

Para todo $k \leqslant N$ e $i=1,2, \ldots, r$, note que o intervalo $\left[t_{i-1}, t_{i}\right)$ está contido em $\left[t_{i^{\prime}-1}^{k}, t_{i^{\prime}}^{k}\right)$ para algum $i^{\prime}$. Dessa forma $w_{k}\left[t_{i-1}, t_{i}\right) \leqslant w_{k}\left[t_{i^{\prime}-1}^{k}, t_{i^{\prime}}^{k}\right)<\epsilon / 2$.

Portanto podemos concluir:

$$
\begin{aligned}
w\left[t_{i-1}, t_{i}\right) & \leqslant \sum_{k=1}^{N} \frac{1}{2^{k}} w_{k}\left[t_{i-1}, t_{i}\right)+\sum_{k>N} \frac{1}{2^{k}} w_{k}\left[t_{i-1}, t_{i}\right) \\
& \leqslant \frac{\epsilon}{2} \sum_{k=1}^{N} \frac{1}{2^{k}}+\sum_{k>N} \frac{1}{2^{k}} \sup _{x, y \in \overline{\mathbb{N}}_{*}} \rho_{0}(x, y) \\
& \leqslant \frac{\epsilon}{2}+\sum_{k>N} \frac{1}{2^{k}} \leqslant \epsilon .
\end{aligned}
$$

Teorema 4.15. Sob a condição de não trivialidade (3.13). Vale que $X_{k} \stackrel{k \rightarrow \infty}{\longrightarrow} \mathbb{Y}$ em probabilidade na topologia de Skorohod usando $\rho$.

Demonstração. Tome qualquer sequência crescente $\left(b_{n}\right)$ de números naturais. Vamos mostrar que essa sequência contem uma sub-sequência $\left(k_{n}\right)_{n}$ sobre a qual $X_{k_{n}}$ converge em probabilidade para $\mathbb{Y}$ quando $n \rightarrow \infty$. Isso implica que $X_{k}$ converge em probabilidade para $\mathbb{Y}$.

Teorema 4.5 garante que para cada $j$ existe uma sub-sequência $\left(a_{n}\right)$ de $\left(b_{n}\right)$ tal que $\theta_{j}^{a_{n}}-\theta_{j}^{\infty}$ converge quase certamente na norma uniforme. Usando o método da diagonal de Cantor, podemos mostrar que existe uma sub-sequência $\left(k_{n}\right)$ de $\left(b_{n}\right)$ tal que $\theta_{j}^{k_{n}}-\theta_{j}^{\infty}$ converge quase certamente para todo $j$. Fixe uma tal sub-sequência. É sobre ela que vamos mostrar a convergência.

Fixe uma realização do processo, vamos mostrar que para quase todas essas realizações e todo $T>0$, existe uma sequência $\left(\lambda_{n}\right)$ de funções, onde $\lambda_{n}:[0, \infty) \rightarrow[0, \infty)$ é uma função estritamente crescente e Lipschitz contínua, que satisfaz:

$$
\begin{array}{r}
\lim _{n \rightarrow \infty} \sup _{t \in[0, T]} \rho\left(X_{k_{n}}(t), \mathbb{Y}\left(\lambda_{n}(t)\right)\right)=0, \\
\lim _{n \rightarrow \infty} \sup _{t \in[0, T]}\left|\lambda_{n}(t)-t\right|=0 .
\end{array}
$$

O Teorema 5.3 do Chapter 3 de [EK86] garante que essas condições são suficientes para mostrar que $X_{k_{n}}$ converge para $\mathbb{Y}$ na topologia de Skorohod.

Vamos mostrar que, para todo $\epsilon>0$, existe uma sequência $\left(\lambda_{n}\right)$ que satisfaz (4.18) e que a quantidade em (4.17) é menor do que $\epsilon$. Isso vai implicar que existe uma sequência $\left(\lambda_{n}\right)$ que satisfaz (4.17) e (4.18).

Para um $\epsilon>0$ arbitrariamente, tome $N, M \in \mathbb{N}_{*}$ tais que $\sum_{j \geqslant N} \frac{1}{2^{j}}<\epsilon / 2$ e $1 /(M+1)<\epsilon / 2$. Suponha que $n$ é grande o suficiente de modo que $k_{n}>N$.

Para construir $\lambda_{n}$, para $k_{n}>N$, tome $S_{n}:=\left\{\theta_{j}^{N}\left(\sigma_{i}^{j, x}-\right), \theta_{j}^{N}\left(\sigma_{i}^{j, x}\right): x \leqslant M, j<N, \theta_{j}^{k_{n}}\left(\sigma_{i}^{j, x}-\right) \leqslant\right.$ $T\}$. Defina $\lambda_{n}$ tal que para todo $s \in S_{n}$ :

$$
\lambda_{n}\left(\theta_{N+1}^{k_{n}}(s)\right)=\theta_{N+1}^{\infty}(s)
$$

Complete $\lambda_{n}$ linearmente entre os pontos de $S_{n}$ e a deixe evoluir linearmente com coeficiente 
angular 1 depois do último ponto. Note que, como $S_{n}$ é finito e $\lambda_{n}$ é linear por partes, então ela é Lipschitz contínua.

O Teorema 3.8 garante que $\lambda_{n}$ é estritamente crescente, então ela é uma candidata para distorção temporal. A Proposição 2.8 nos diz que a $j$-ésima coordenada, $j<N$, de $X_{k_{n}}(t)$ é igual à $x \leqslant M$ se e somente se:

$$
\begin{array}{r}
t \in \bigcup_{i=1}\left[\theta_{j}^{k_{n}}\left(\sigma_{i}^{j, x}-\right), \theta_{j}^{k_{n}}\left(\sigma_{i}^{j, x}\right)\right)=\bigcup_{i=1}\left[\theta_{N+1}^{k_{n}}\left(\theta_{j}^{N}\left(\sigma_{i}^{j, x}-\right)\right), \theta_{N+1}^{k_{n}}\left(\theta_{j}^{N}\left(\sigma_{i}^{j, x}\right)\right)\right) \Leftrightarrow \\
\lambda_{n}(t) \in \bigcup_{i=1}\left[\theta_{N+1}^{\infty}\left(\theta_{j}^{N}\left(\sigma_{i}^{j, x}-\right)\right), \theta_{N+1}^{\infty}\left(\theta_{j}^{N}\left(\sigma_{i}^{j, x}\right)\right)\right)=\bigcup_{i=1}\left[\theta_{j}^{\infty}\left(\sigma_{i}^{j, x}-\right), \theta_{N+1}^{\infty}\left(\sigma_{i}^{j, x}\right)\right) .
\end{array}
$$

Observe que usamos o Corolário 4.8 na última passagem. Com isso concluímos que, para qualquer coordenada $j<N$ :

$$
\begin{aligned}
\sup _{t \in[0, T]} \rho_{0}\left(X_{k_{n}, j}(t), Y_{j}\left(\lambda_{n}(t)\right)\right) & \leqslant \frac{1}{M+1}<\frac{\epsilon}{2} \\
\sup _{t \in[0, T]} \rho\left(X_{k_{n}}(t), \mathbb{Y}\left(\lambda_{n}(t)\right)\right) & <\frac{\epsilon}{2}+\sum_{j \geqslant N} \frac{1}{2^{j}}<\epsilon .
\end{aligned}
$$

Isso encerra a prova de (4.17). Para provar (4.18) tome $T^{\prime}>0$ tal que $\theta_{j}^{\infty}\left(T^{\prime}\right)>T+1$ para todo $j<N$ e note que, para $n$ grande o suficiente:

$$
\sup _{t \in[0, T]}\left|\lambda_{n}(t)-t\right|=\max _{s \in S_{n}}\left|\theta_{N+1}^{k_{n}}(s)-\theta_{N+1}^{\infty}(s)\right| \leqslant \sup _{s \in\left[0, T^{\prime}\right]}\left|\theta_{N+1}^{k_{n}}(s)-\theta_{N+1}^{\infty}(s)\right| \stackrel{n \rightarrow \infty}{\longrightarrow} 0 .
$$

\subsection{Medida Empírica}

Vamos assumir toda essa seção que o meio aleatório $\gamma=\left\{\gamma_{k}\left(\left.x\right|_{k}\right): k \in \mathbb{N}_{*},\left.x\right|_{k} \in \mathbb{N}_{*}^{k}\right\}$ está fixado. Todos os resultados dessa seção valem para quase todas as escolhas do meio aleatório.

Nessa seção iremos calcular a medida empírica assintótica do Processo K em árvores de profundidade infinita, isso é, a proporção do tempo que o processo título passa em cilindros $\left[\left.x\right|_{k}\right]=\left\{\left.y\right|_{\infty} \in\right.$ $\left.\overline{\mathbb{N}}_{*}^{\mathbb{N} *}:\left.y\right|_{k}=\left.x\right|_{k}\right\}$. Provaremos que ela é dada por:

$$
\lim _{t \rightarrow \infty} \frac{1}{t} \int_{0}^{\infty} \mathbb{I}\left\{\mathbb{Y}(t) \in\left[\left.x\right|_{k}\right]\right\} d t \underset{\text { q.c. }}{\stackrel{t \rightarrow \infty}{\longrightarrow}} \frac{\bar{\gamma}_{k}\left(\left.x\right|_{k}\right) \mathbb{E}_{\gamma}\left[\theta_{\left.x\right|_{k}}^{\infty}(1)\right]}{\mathbb{E}_{\gamma}\left[\theta_{1}^{\infty}(1)\right]}
$$

Note que provamos que essa quantidade é finita no Teorema 3.6. Vamos precisar da seguinte conjectura:

Conjectura 4.1. O Processo $K$ em uma árvore de profundidade infinita é fortemente Markoviano.

Pensamos que é possível mostrar esse resultado usando uma técnica análoga à empregada em Fontes e Mathieu [FM08] e Fontes e Peixoto [FP13], onde os autores aproximam o processo título de seus artigos por processos que são markovianos, e provam que a propriedade de Markov sobrevive ao limite.

Duas complicações aparecem ao tentar utilizar esse argumento. A primeira é que não temos uma prova de que os Processos K em árvores de profundidade finita são markovianos. 
Uma segunda complicação é que o argumento usa de maneira forte que o processo limite tenha a propriedade de Feller, algo que preferimos evitar nesse trabalho, já que não construímos um Processo K iniciado em um estado $y \in \overline{\mathbb{N}}_{*}^{\mathbb{N} *}$ arbitrário.

Dessa forma deixamos esse problema para um trabalho futuro, e vamos assumir a Conjectura 4.1 pelo restante da seção.

Definição 4.2. Vamos denotar as primeiras $k$ coordenadas do processo $\mathbb{Y}$ por $\left.Y\right|_{k}$, isso é:

$$
\left.Y\right|_{k}:=\left(Y_{1}, \ldots, Y_{k}\right)
$$

Proposição 4.16. Se T é uma variável aleatória positiva, independente do processo $\theta_{\left.x\right|_{k}}^{\infty}$ então:

$$
\mathbb{E}_{\gamma}\left[\theta_{\left.x\right|_{k}}^{\infty}(T)\right]=\mathbb{E}_{\gamma}\left[\theta_{\left.x\right|_{k}}^{\infty}(1)\right] \mathbb{E}(T)
$$

Demonstração. Vamos provar esse resultado para $\theta_{1}^{\infty}$, a Proposição 3.4 estende essa prova para o caso geral.

Fixe $n, m \in \mathbb{N}_{*}$ arbitrariamente, já que $\theta_{1}^{\infty}$ é um subordinador (Lema 4.4) então $\theta_{1}^{\infty}(n / m)$ tem a mesma lei que a soma de $n$ cópias independentes de $\theta_{1}^{\infty}(1 / m)$. Pelo mesmo argumento $\theta_{1}^{\infty}(1)$ tem a mesma distribuição que a soma de $m$ cópias independentes de $\theta_{1}^{\infty}(1 / m)$. Dessa forma:

$$
\mathbb{E}_{\gamma}\left[\theta_{1}^{\infty}\left(\frac{n}{m}\right)\right]=n \mathbb{E}_{\gamma}\left[\theta_{1}^{\infty}\left(\frac{1}{m}\right)\right]=\frac{n}{m} \mathbb{E}_{\gamma}\left[\theta_{1}^{\infty}(1)\right] .
$$

Para um $t>0$ real arbitrário, tome $q, r$ números racionais tais que $0<q<t<r$. Como um subordinador é monótono, então:

$$
\begin{aligned}
& \mathbb{E}_{\gamma}\left[\theta_{1}^{\infty}(q)\right] \leqslant \mathbb{E}_{\gamma}\left[\theta_{1}^{\infty}(t)\right] \leqslant \mathbb{E}_{\gamma}\left[\theta_{1}^{\infty}(r)\right] \\
\Leftrightarrow & q \mathbb{E}_{\gamma}\left[\theta_{1}^{\infty}(1)\right] \leqslant \mathbb{E}_{\gamma}\left[\theta_{1}^{\infty}(t)\right] \leqslant r \mathbb{E}_{\gamma}\left[\theta_{1}^{\infty}(1)\right] .
\end{aligned}
$$

Já que $q, r$ podem ser tomados arbitrariamente próximos de $t$ então $\mathbb{E}\left[\theta_{1}^{\infty}(t)\right]=t\left[\theta_{1}^{\infty}(1)\right]$. Para completar a prova, denote por $\nu$ a medida de probabilidade associada com $T$. Como $T$ é independente de $\theta_{1}^{\infty}$, concluímos que:

$$
\begin{aligned}
\mathbb{E}_{\gamma}\left[\theta_{1}^{\infty}(T)\right] & =\int \mathbb{E}_{\gamma}\left[\theta_{1}^{\infty}(t)\right] \nu(d t) \\
& =\mathbb{E}_{\gamma}\left[\theta_{1}^{\infty}(1)\right] \int t \nu(d t) \\
& =\mathbb{E}_{\gamma}\left[\theta_{1}^{\infty}(1)\right] \mathbb{E}(T) .
\end{aligned}
$$

O próximo resultado nos diz que, para um $t \geqslant 0$ fixado, a família $\left\{\mathbb{E}_{\gamma}\left[\theta_{\left.x\right|_{k}}^{\infty}(t)\right]: k \in \mathbb{N}_{*},\left.x\right|_{k} \in \mathbb{N}_{*}^{k}\right\}$ satisfaz uma lei de composição análoga à observada na Proposição 3.3 para a família $\left\{W\left(\left.x\right|_{k}\right): k \in\right.$ $\left.\mathbb{N}_{*},\left.x\right|_{k} \in \mathbb{N}_{*}^{k}\right\}$. 
Proposição 4.17. Para quaisquer $t>0$ e $\left.x\right|_{k} \in \mathbb{N}_{*}^{k}$ fixados, se $\sum_{i}\left(1-\alpha_{i}\right)<\infty$ então:

$$
\begin{aligned}
\mathbb{E}_{\gamma}\left[\theta_{\left.x\right|_{k}}^{\infty}(t)\right] & =\sum_{x_{k+1}} \gamma_{k+1}\left(\left.x\right|_{k+1}\right) \mathbb{E}_{\gamma}\left[\theta_{\left.x\right|_{k+1}}^{\infty}(t)\right] \\
\mathbb{E}_{\gamma}\left[\theta_{\left.x\right|_{k}}^{\infty}\left(\sigma_{1}^{k+1, x_{k+1}}-\right)\right] & =\mathbb{E}_{\gamma}\left[\theta_{\left.x\right|_{k}}^{\infty}(1)\right]-\gamma_{k+1}\left(\left.x\right|_{k+1}\right) \mathbb{E}_{\gamma}\left[\theta_{\left.x\right|_{k+1}}^{\infty}(1)\right] .
\end{aligned}
$$

Demonstração. Vamos provar apenas o caso $\left.x\right|_{k}=\varnothing(k=0)$. Para mostrar (4.20) vamos aplicar o Corolário 4.8 para quebrar a contribuição cada possível primeira coordenada e depois apliquemos a Proposição 4.16:

$$
\begin{aligned}
\mathbb{E}_{\gamma}\left[\theta_{1}^{\infty}(t)\right] & =\mathbb{E}_{\gamma}\left[\theta_{2}^{\infty}\left(\Xi_{1}(t)\right)\right] \\
& =\mathbb{E}_{\gamma}\left[\theta_{2}^{\infty}\left(\sum_{x_{1}} \sum_{i=1}^{N^{1, x_{1}}(t)} \gamma_{1}\left(\left.x\right|_{1}\right) T_{i}^{1, x_{1}}\right)\right] \\
& =\mathbb{E}_{\gamma}\left[\sum_{x_{1}} \theta_{\left.x\right|_{1}}^{\infty}\left(\sum_{i=1}^{N^{1, x_{1}}(t)} \gamma_{1}\left(\left.x\right|_{1}\right) T_{i}^{1, x_{1}}\right)\right] \\
& =\mathbb{E}_{\gamma}\left[\sum_{x_{1}} \theta_{\left.x\right|_{1}}^{\infty}\left(\sum_{i=1}^{N^{1, x_{1}}(t)} \gamma_{1}\left(\left.x\right|_{1}\right) T_{i}^{1, x_{1}}\right)\right] \\
& =\sum_{x_{1}} \mathbb{E}_{\gamma}\left[\theta_{\left.x\right|_{1}}^{\infty}(1)\right] \mathbb{E}_{\gamma}\left[\sum_{i=1}^{N^{1, x_{1}}(t)} \gamma_{1}\left(\left.x\right|_{1}\right) T_{i}^{1, x_{1}}\right] \\
& =\sum_{x_{1}} \mathbb{E}_{\gamma}\left[\theta_{\left.x\right|_{1}}^{\infty}(1)\right] t \gamma_{1}\left(\left.x\right|_{1}\right) \\
& =\sum_{x_{1}} \mathbb{E}_{\gamma}\left[\theta_{\left.x\right|_{1}}^{\infty}(t)\right] \gamma_{1}\left(\left.x\right|_{1}\right) .
\end{aligned}
$$

Para provar (4.21), note que a distribuição de $\theta_{\left.x\right|_{k}}^{\infty}$, até o instante $\sigma_{i}^{k+1, x_{k+1}}$, é independente do valor desse instante, com a restrição de que o processo não encontra nenhum ponto originário do Processo de Poisson $N^{k+1, x_{k+1}}$. Isso seria equivalente a forçar $\gamma_{k+1}\left(\left.x\right|_{k+1}\right)=0$.

Se denotarmos por $\hat{\theta}_{\left.x\right|_{k}}^{\infty}$ uma versão de $\theta_{\left.x\right|_{k}}^{\infty}$ onde fazemos essa modificação, podemos utilizar o resultado anterior e a Proposição 4.16 para obter:

$$
\begin{aligned}
\mathbb{E}_{\gamma}\left[\theta_{\left.x\right|_{k}}^{\infty}\left(\sigma_{1}^{k+1, x_{k+1}}-\right)\right] & =\mathbb{E}_{\gamma}\left[\hat{\theta}_{\left.x\right|_{k}}^{\infty}\left(\sigma_{1}^{k+1, x_{k+1}}\right)\right] \\
& =\mathbb{E}_{\gamma}\left(\sigma_{1}^{k+1, x_{k+1}}\right) \mathbb{E}_{\gamma}\left[\hat{\theta}_{\left.x\right|_{k}}^{\infty}(1)\right] \\
& =\sum_{y \neq x_{k+1}} \gamma_{k+1}\left(\left.x\right|_{k} y\right) \mathbb{E}_{\gamma}\left[\theta_{\left.x\right|_{k} y}^{\infty}(1)\right] \\
& =\mathbb{E}_{\gamma}\left[\theta_{\left.x\right|_{k}}^{\infty}(1)\right]-\gamma_{k+1}\left(\left.x\right|_{k+1}\right) \mathbb{E}_{\gamma}\left[\theta_{\left.x\right|_{k+1}}^{\infty}(1)\right]
\end{aligned}
$$

Definição 4.3. Para um $\left.y\right|_{k} \in \mathbb{N}_{*}^{k}$ fixado, vamos denotar por $U_{i}$ o instante da i-ésima entrada de $\left.Y\right|_{k}$ em $\left.y\right|_{k}$. Analogamente vamos chamar a $i$-ésima saída de $V_{i}$. Isso é, definimos $V_{0}:=0$ e para 
$i=1,2, \ldots$

$$
\begin{aligned}
& U_{i}:=\inf \left\{t>V_{i-1}:\left.Y\right|_{k}(t)=\left.y\right|_{k}\right\}, \\
& V_{i}:=\inf \left\{t>U_{i}:\left.Y\right|_{k}(t) \neq\left. y\right|_{k}\right\} .
\end{aligned}
$$

Proposição 4.18. No caso não trivial, para todo $i$ vale que:

$$
Y_{j}\left(U_{i}\right)=\left\{\begin{array}{ll}
y_{j} & \text { se } j \leqslant k \\
\infty & \text { caso contrário, }
\end{array} \quad Y_{j}\left(V_{i}\right)= \begin{cases}y_{j} & \text { se } j<k \\
\infty & \text { caso contrário. }\end{cases}\right.
$$

Demonstração. Por construção, sabemos que $U_{i}=\theta_{k}^{\infty}\left(\sigma_{j}^{k, y_{k}}-\right)$ e $V_{i}=\theta_{k}^{\infty}\left(\sigma_{j}^{k, y_{k}}\right)$ para algum $j$.

Dessa forma $V_{i}$ pertence à imagem de $\theta_{k}^{\infty}$, de onde o Corolário 4.10 nos afirma que $Y_{k}\left(V_{i}\right)=\infty$. Agora podemos aplicar o Corolário 4.11 para concluir que $Y_{j}\left(V_{i}\right)=\infty$ para todo $j \geqslant k$.

A Proposição 2.12 nos diz que existe $j^{\prime}$ tal que $\sigma_{j}^{k, y_{k}} \in\left[\Xi_{k-1}\left(\sigma_{j^{\prime}}^{k-1, y_{k-1}}-\right), \Xi_{k-1}\left(\sigma_{j^{\prime}}^{k-1, y_{k-1}}\right)\right)$, dessa forma temos que:

$$
\begin{gathered}
\Xi_{k-1}\left(\sigma_{j^{\prime}}^{k-1, y_{k-1}}-\right) \leqslant \sigma_{j}^{k, y_{k}}<\Xi_{k-1}\left(\sigma_{j^{\prime}}^{k-1, y_{k-1}}\right) \\
\Leftrightarrow \theta_{k-1}^{\infty}\left(\sigma_{j^{\prime}}^{k-1, y_{k-1}}-\right) \leqslant \theta_{k}^{\infty}\left(\sigma_{j}^{k, y_{k}}\right)=V_{i}<\theta_{k-1}^{\infty}\left(\sigma_{j^{\prime}}^{k-1, y_{k-1}}\right) .
\end{gathered}
$$

Dessa forma $V_{i}$ pertence a um intervalo onde $Y_{k-1}$ é constante e igual à $y_{k-1}$. Como esses intervalos são encaixados (Proposição 2.12), então concluímos que $Y_{j}\left(V_{i}\right)=y_{j}$ para todo $j<k$.

Como $\mathbb{Y}$ é contínuo à direita (Proposição 4.14) então vale que $Y_{j}\left(U_{i}\right)=y_{j}$ para $j \leqslant k$.

Lembre que $U_{i}=\theta_{k}^{\infty}\left(\sigma_{j}^{k, y_{k}}-\right)$ e suponha, por absurdo, que $Y_{k+1}\left(U_{i}\right)=x<\infty$. Nesse caso existe um $\sigma_{i^{\prime}}^{k+1, x}$ tal que:

$$
\begin{gathered}
\theta_{k+1}^{\infty}\left(\sigma_{i^{\prime}}^{k+1, x}-\right) \leqslant U_{i}<\theta_{k+1}^{\infty}\left(\sigma_{i^{\prime}}^{k+1, x}\right) \\
\Leftrightarrow \theta_{k+1}^{\infty}\left(\sigma_{i^{\prime}}^{k+1, x}-\right) \leqslant \theta_{k}^{\infty}\left(\sigma_{j}^{k, y_{k}}-\right)<\theta_{k+1}^{\infty}\left(\sigma_{i^{\prime}}^{k+1, x}\right) \\
\Leftrightarrow \theta_{k+1}^{\infty}\left(\sigma_{i^{\prime}}^{k+1, x}-\right) \leqslant \theta_{k+1}^{\infty}\left(\Xi_{k}\left(\sigma_{j}^{k, y_{k}}-\right)\right)<\theta_{k+1}^{\infty}\left(\sigma_{i^{\prime}}^{k+1, x}\right) .
\end{gathered}
$$

Como $\theta_{k+1}^{\infty}$ é estritamente crescente, então a primeira desigualdade da última expressão implica que $\sigma_{i^{\prime}}^{k+1, x} \leqslant \Xi_{k}\left(\sigma_{j}^{k, y_{k}}-\right)$. Por outro lado a segunda desigualdade implica que $\Xi_{k}\left(\sigma_{j}^{k, y_{k}}-\right)<\sigma_{i^{\prime}}^{k+1, x}$. Aqui encontramos uma contradição, de onde concluímos que $Y_{k+1}\left(U_{i}\right)=\infty$. Aplicando o Corolário 4.11 concluiremos que $Y_{j}\left(U_{i}\right)=\infty$ para todo $j>k$.

O incremento $V_{i}-U_{i}$ é o tempo que o processo $\mathbb{Y}$ passa em $\left.y\right|_{k}$ na sua i-ésima visita. Ele é igual à $\theta_{k}^{\infty}\left(\sigma_{j}^{k, y_{k}}\right)-\theta_{k}^{\infty}\left(\sigma_{j}^{k, y_{k}}-\right)$ para algum $j$. Dessa forma $\mathbb{E}\left(V_{i}-U_{i}\right)=\mathbb{E}\left[\theta_{\left.y\right|_{k}}^{\infty}\left(\gamma_{k}\left(\left.x\right|_{k}\right) T_{1}^{k, y_{k}}\right)\right]=$ $\gamma_{k}\left(\left.y\right|_{k}\right) \mathbb{E}\left(\theta_{\left.y\right|_{k}}^{\infty}(1)\right)$.

Proposição 4.19. O incremento $U_{i+1}-V_{i}$ é o tempo entre visitas consecutivas à $\left.y\right|_{k}$. O seu valor esperado pode ser calculado como:

$$
\mathbb{E}_{\gamma}\left(U_{i+1}-V_{i}\right)=\frac{\mathbb{E}_{\gamma}\left(\theta_{1}^{\infty}(1)\right)}{\bar{\gamma}_{k-1}\left(\left.y\right|_{k-1}\right)}-\gamma_{k}\left(\left.y\right|_{k}\right) \mathbb{E}_{\gamma}\left(\theta_{\left.y\right|_{k}}^{\infty}(1)\right)
$$

sob a convenção de que $\bar{\gamma}_{0}\left(\left.y\right|_{0}\right):=1$. 
Demonstração. Vamos denotar por $S_{j}^{1}, S_{j}^{2}, \ldots$ os intervalos entre visitas consecutivas à $\left.y\right|_{j}$. A propriedade forte de Markov (Conjectura 4.1) nos diz que essas variáveis formam uma sequência i.i.d.. Vamos denotar ainda por $a_{j}:=\mathbb{E}\left(S_{j}^{1}\right)$. Queremos calcular $a_{k}$.

No instante $V_{i}$ o processo acabou de sair de $\left.y\right|_{k}$, isso é, $V_{i}=\theta_{k}^{\infty}\left(\sigma_{i^{\prime}}^{k, y_{k}}\right)$ para algum $i^{\prime}$. Existe um $\sigma_{i^{\prime \prime}}^{k-1, y_{k-1}}$ tal que $\sigma_{i^{\prime}}^{k, y_{k}} \in\left[\Xi_{k-1}\left(\sigma_{i^{\prime \prime}}^{k-1, y_{k-1}}-\right), \Xi_{k-1}\left(\sigma_{i^{\prime \prime}}^{k-1, y_{k-1}}\right)\right)$. Esse intervalo tem comprimento $\gamma_{k-1}\left(\left.y\right|_{k-1}\right) T_{i^{\prime \prime}}^{k-1, y_{k-1}}$. Por causa da falta de memória da distribuição exponencial, a lei de $\Xi_{k-1}\left(\sigma_{i^{\prime \prime}}^{k-1, y_{k-1}}\right)-$ $\sigma_{i^{\prime}}^{k, y_{k}}$ é a mesma que a lei do comprimento total do intervalo.

Com probabilidade $p=\gamma_{k-1}\left(\left.y\right|_{k-1}\right) /\left(1+\gamma_{k-1}\left(\left.y\right|_{k-1}\right)\right)$ vamos observar $\left.\sigma_{i^{\prime}+1}^{k, y_{k}}<\Xi_{k-1}\left(\sigma_{i^{\prime \prime}}^{k-1, y_{k-1}}\right)\right)$. Nesse caso o Processo K vai visitar $\left.y\right|_{k}$ antes de sair de $\left.y\right|_{k-1}$.

Se isso não acontecer, vai levar um tempo com lei $S_{k-1}^{1}$ para que o Processo K visite $\left.y\right|_{k-1}$ novamente, nessa ocasião teremos uma probabilidade $p$ de visitarmos $\left.y\right|_{k}$ durante essa visita a $\left.y\right|_{k-1}$. Caso isso haja uma segunda falha então o processo esperará $S_{k-1}^{2}$ para uma terceira tentativa e assim por diante.

Portanto o tempo entre visitas consecutivas a $\left.y\right|_{k}$ que o processo passa fora de $\left.y\right|_{k-1}$ tem a mesma lei que $\sum_{i=1}^{M} S_{k-1}^{i}$, onde $M$ é uma variável geométrica com probabilidade de sucesso $p$, independente de $S_{k-1}^{i}, i=1,2, \ldots$

O tempo total passado em $\left.y\right|_{k-1}$, mas fora de $\left.y\right|_{k}$ tem a mesma lei que $\theta_{\left.y\right|_{k-1}}\left(\sigma_{1}^{k, y_{k}}-\right)$. Dessa forma, usando a Proposição 4.17:

$$
\begin{aligned}
a_{k} & =a_{k-1} \frac{1-p}{p}+\mathbb{E}_{\gamma}\left[\theta_{\left.y\right|_{k-1}}\left(\sigma_{1}^{k, y_{k}}-\right)\right] \\
& =\frac{a_{k-1}}{\gamma_{k-1}\left(\left.y\right|_{k-1}\right)}+\mathbb{E}_{\gamma}\left[\theta_{\left.y\right|_{k-1}}^{\infty}(1)\right]-\gamma_{k}\left(\left.y\right|_{k}\right) \mathbb{E}_{\gamma}\left[\theta_{\left.y\right|_{k}}^{\infty}(1)\right] .
\end{aligned}
$$

Dessa forma obtemos uma recorrência em $k$ para $a_{k}$, que podemos usar para provar (4.23) com um argumento de indução.

A propriedadade forte de Markov (Conjectura 4.1) nos diz que os incrementos das variáveis $\left(U_{i}, V_{i}\right)$ são independentes. Portanto podemos usar a lei forte dos grandes números para calcular a proporção do tempo que o Processo K passa em um estado $\left.y\right|_{k}$ :

$$
\begin{aligned}
\pi\left(\left.y\right|_{k}\right) & :=\lim _{n \rightarrow \infty} \frac{\sum_{i=1}^{\infty} V_{i}-U_{i}}{V_{n}} \stackrel{\text { q.c. }}{=} \frac{\mathbb{E}_{\gamma}\left[V_{i}-U_{i}\right]}{\mathbb{E}_{\gamma}\left[U_{i+1}-U_{i}\right]}=\frac{\mathbb{E}_{\gamma}\left[V_{i}-U_{i}\right]}{\mathbb{E}_{\gamma}\left[U_{i+1}-V_{i}\right]+\mathbb{E}_{\gamma}\left[V_{i}-U_{i}\right]} \\
& =\frac{\bar{\gamma}_{k}\left(\left.y\right|_{k}\right) \mathbb{E}_{\gamma}\left[\theta_{\left.y\right|_{k}}^{\infty}(1)\right]}{\mathbb{E}_{\gamma}\left[\theta_{1}^{\infty}(1)\right]} .
\end{aligned}
$$

Note que chegamos na mesma fórmula que anunciamos em (4.19). Podemos usar o teorema da consistência de Kolmogorov para provar que essa função $\pi$ define uma medida sobre a $\sigma$-álgebra produto de $\mathbb{N}_{*}^{\mathbb{N} *}$. A Proposição 4.17 nos diz ainda que ela é uma medida de probabilidade.

Na prova do Teorema 3.6, obtemos que:

$$
\mathbb{E}_{\gamma}\left[\theta_{\left.x\right|_{k}}^{\infty}(t)\right] \leqslant \liminf _{n \rightarrow \infty} \mathbb{E}_{\gamma}\left[\widetilde{\theta}_{\left.x\right|_{k}}^{n}(t)\right]=t W\left(\left.x\right|_{k}\right)
$$

Não sabemos calcular $\mathbb{E}_{\gamma}\left[\theta_{1}^{\infty}(t)\right]$. Mas não nos surpreenderíamos se a desigualdade da equação anterior fosse de fato uma igualdade. Isso forneceria uma interpretação interessante para a probabilidade $\pi$ em termos do meio aleatório $\left\{\gamma_{k}\left(\left.x\right|_{k}\right)\right\}$. Ela seria proporcional à $\bar{\gamma}_{k}\left(\left.y\right|_{k}\right) W\left(\left.y\right|_{k}\right)$, dividida 
por um fator de normalização. 


\section{Capítulo 5}

\section{Conclusões}

Nesse trabalho introduzimos o Processo K em uma árvore de profundidade infinita, demos condições em que nossa construção resulta em um processo não trivial e, sob essas condições, provamos que ele é o limite dos Processos $\mathrm{K}$ em árvores de profundidade infinita, introduzidas por Fontes et al. [FGG14], quando a altura das árvores cresce para o infinito.

Para um estudo futuro, podemos sugerir provar que o processo título é fortemente markoviano, como indicado na Seção 4.5.

Outra pergunta em aberto diz respeito à escolha das constantes $c_{k}$ feita em (2.1). O processo limite só existiu porque fizemos essa escolha particular. Podemos interpretar o Teorema 4.15 como uma espécie de limite de escala, com a escala definida indiretamente pela nossa escolha das constantes.

Mais especificamente, quanto poderíamos relaxar a escolha dessas constantes e ainda assim obter o mesmo processo limite? Será que existe alguma escolha que resulte em outro processo limite?

Fontes et al. [FGG14] mostraram que, sob certas condições, o Processo K em árvores de profundidade finita é limite de escala de modelos de armadilha estilo GREM, quando os volumes das árvores crescem de uma maneira controlada. Como mostramos que o nosso processo título é limite de Processos $\mathrm{K}$ de profundidade finita, então podemos provar que o Processo $\mathrm{K}$ em árvores de profundidade infinita também pode ser obtido como limite de modelos de armadilha estilo GREM. Porém para isso usamos um argumento abstrato que não nos dá critérios concretos sobre como o volume de cada nível da árvore deve crescer em relação à velocidade de crescimento da altura da árvore. Encontrar tais condições seria outra pergunta para um trabalho futuro. 
CONCLUSÕES 


\section{Apêndice A}

\section{Resultados auxiliares}

Esse apêndice traz resultados auxiliares que tratam sobre Processos de Poisson, distribuições estáveis e transformadas de Laplace.

Proposição A.1. Seja $\left\{\gamma_{i}: i \in \mathbb{N}\right\}$ as marcas de um Processo Pontual de Poisson em $\mathbb{R}^{+}$com medida intensidate $\mu(d t)=c / t^{1+\alpha}$, com $t>0, \alpha \in(0,1)$ e $c>0$. Sejam $\left\{X_{i}: i \in \mathbb{N}\right\}$ uma família de variáveis aleatórias i.i.d., positivas e tomadas de maneira independente do Processo de Poisson, tais que $\mathbb{E}\left(X_{1}^{\alpha}\right)<\infty$.

Nessas condições $\left\{\gamma_{i} X_{i}: i \in \mathbb{N}\right\}$ é um Processo Pontual de Poisson com medida intensidade $\mathbb{E}\left(X_{1}^{\alpha}\right) \mu$.

Demonstração. Tome $S:=\left\{\left(\gamma_{i}, X_{i}\right): i \in \mathbb{N}\right\}$ e note que $S$ corresponde às marcas de um Processo Pontual de Poisson no primeiro quadrante de $\mathbb{R}^{2}$, com medida intensidade $\pi=\mu \times \nu$, onde $\nu$ é a medida de probabilidade associada à $X_{1}$.

Como $T(x, y)=x y$ é uma transformação contínua sem pontos de acumulação fora da origem, segue que $\{T(s): s \in S\}=\left\{\gamma_{i} X_{i}: i \in \mathbb{N}\right\}$ é um Processo Pontual de Poisson [veja Kin93, Mapping Theorem, seção 2.3]. Para calcular sua medida intensidade, tome $0<a<b<\infty$ e perceba que:

$$
\begin{aligned}
\pi\left(\left\{(x, y) \in \mathbb{R}_{+}^{2}: T(x, y) \in(a, b)\right\}\right) & =\int_{0}^{\infty} \mu\left(\left\{x \in \mathbb{R}_{+}: x y \in(a, b)\right\}\right) \nu(d y) \\
& =\int_{0}^{\infty} \int_{a / y}^{b / y} \frac{c}{x^{1+\alpha}} d x \nu(d y) \\
& =\int_{0}^{\infty} c\left(\frac{y^{\alpha}}{a^{\alpha}}-\frac{y^{\alpha}}{b^{\alpha}}\right) \nu(d y) \\
& =c\left(\frac{1}{a^{\alpha}}-\frac{1}{b^{\alpha}}\right) \int_{0}^{\infty} y^{\alpha} \nu(d y) \\
& =\mu(a, b) \mathbb{E}\left[X_{1}^{\alpha}\right] .
\end{aligned}
$$

Proposição A.2. Se $X$ é uma variável aleatória positiva, com transformada de Laplace $\phi(\lambda):=$ $\mathbb{E}\left(e^{-\lambda X}\right)$. Então para todo $\beta \in(0,1)$ :

$$
\mathbb{E}\left(X^{\beta}\right)=-\frac{1}{\Gamma(1-\beta)} \int_{0}^{\infty} \phi^{\prime}(\lambda) \lambda^{-\beta} d \lambda
$$

Demonstração. Um resultado clássico nos diz que $\phi^{\prime}(\lambda)=-\mathbb{E}\left[X e^{-\lambda X}\right]$. Podemos verificar esse 
resultado calculando explicitamente a derivada de $\phi$ :

$$
\begin{aligned}
\phi^{\prime}(\lambda) & =\lim _{h \rightarrow 0} \frac{\phi(\lambda+h)-\phi(\lambda)}{h} \\
& =\lim _{h \rightarrow 0} \mathbb{E}\left[e^{-\lambda X} \frac{e^{-h X}-1}{h}\right] .
\end{aligned}
$$

O valor absoluto da variável dentro da esperança pode ser limitado por $X e^{-\lambda X} \leqslant \frac{e^{-1}}{\lambda}$. Dessa forma, aplicando o Teorema da Convergência Dominada, concluímos que:

$$
\phi^{\prime}(\lambda)=\mathbb{E}\left[-X e^{-\lambda X}\right]
$$

Agora vamos calcular a integral em (A.1). Como o termo integrado é sempre positivo então podemos aplicar o Teorema de Fubini para trocar a ordem da integral e da esperança. Depois podemos fazer uma mudança de variáveis $v=\lambda X$, obtendo:

$$
\begin{aligned}
-\int_{0}^{\infty} \phi^{\prime}(\lambda) \lambda^{-\beta} d \lambda & =\int_{0}^{\infty} \mathbb{E}\left[X e^{-\lambda X}\right] \lambda^{-\beta} d \lambda \\
& =\mathbb{E}\left[\int_{0}^{\infty} X e^{-\lambda X} \lambda^{-\beta} d \lambda\right] \\
& =\mathbb{E}\left[X^{\beta} \int_{0}^{\infty} e^{-v} v^{-\beta} d v\right] \\
& =\mathbb{E}\left[X^{\beta}\right] \Gamma(1-\beta) .
\end{aligned}
$$

Proposição A.3. Se $X$ é uma variável aleatória positiva, com transformada de Laplace $\phi(\lambda):=$ $e^{-c \lambda^{\alpha}}$, para algum $c>0$ e $\alpha \in(0,1)$. Então para $0<\beta<\alpha$ :

$$
\mathbb{E}\left(X^{\beta}\right)=c^{\beta / \alpha} \frac{\Gamma(1-\beta / \alpha)}{\Gamma(1-\beta)} .
$$

Demonstração. Aplicando a Proposição A.2, obtemos que:

$$
\begin{aligned}
\mathbb{E}\left(X^{\beta}\right) & =-\frac{1}{\Gamma(1-\beta)} \int_{0}^{\infty} \phi^{\prime}(\lambda) \lambda^{-\beta} d \lambda \\
& =\frac{1}{\Gamma(1-\beta)} \int_{0}^{\infty} c \alpha \lambda^{\alpha-1} e^{-c \lambda^{\alpha}} \lambda^{-\beta} d \lambda \\
& =\frac{1}{\Gamma(1-\beta)} \int_{0}^{\infty} e^{-v}\left(\frac{v}{c}\right)^{-\frac{\beta}{\alpha}} d v \\
& =c^{\beta / \alpha} \frac{\Gamma(1-\beta / \alpha)}{\Gamma(1-\beta)} .
\end{aligned}
$$




\section{Bibliografia}

$[$ Bez +12$]$ S. C. Bezerra, L. R. G. Fontes, R. J. Gava, V. Gayrard e P. Mathieu. "Scaling limits and aging for asymmetric trap models on the complete graph and K-processes". Em: ArXiv e-prints (fev. de 2012). arXiv: 1202.4101 [math.PR] (ver p. 1).

[Bou92] J. -P. Bouchaud. "Weak ergodicity breaking and aging in disordered systems". Em: J. Phys. I France 2.9 (set. de 1992), pp. 1705-1713. DOI: 10.1051/jp1:1992238 (ver p. 1).

[BD95] J. -P. Bouchaud e D. S. Dean. "Aging on Parisi's Tree". Em: J. Phys. I France 5.3 (1995), pp. 265-286. DOI: 10.1051/jp1:1995127. URL: http://dx.doi.org/10.1051/jp1:1995127 (ver p. 1).

[Dur10] Rick Durrett. Probability: theory and examples. Fourth. Cambridge Series in Statistical and Probabilistic Mathematics. Cambridge: Cambridge University Press, 2010, pp. $\mathrm{x}+428$. ISBN: 978-0-521-76539-8 (ver pp. 14, 20, 37).

[EK86] Stewart N. Ethier e Thomas G. Kurtz. Markov processes. Wiley Series in Probability and Mathematical Statistics: Probability and Mathematical Statistics. Characterization and convergence. New York: John Wiley \& Sons Inc., 1986, pp. x+534. ISBN: 0-47108186-8. DOI: 10.1002/9780470316658. URL: http://dx.doi.org/10.1002/9780470316658 (ver pp. 33, 41).

[FGG14] L. R. G. Fontes, R. J. Gava e V. Gayrard. "The K-process on a tree as a scaling limit of the GREM-like trap model". Em: The Annals of Applied Probability 24.2 (abr. de 2014), pp. 857-897. DOI: 10.1214/13-AAP937. URL: http://dx.doi.org/10.1214/13-AAP937 (ver pp. iii, v, 1-4, 49).

[FL09] L.R.G. Fontes e P.H.S. Lima. "Convergence of Symmetric Trap Models in the Hypercube". English. Em: New Trends in Mathematical Physics. Ed. por Vladas Sidoravičius. Springer Netherlands, 2009, pp. 285-297. ISBN: 978-90-481-2809-9. DOI: 10.1007/97890-481-2810-5_20. URL: http://dx.doi.org/10.1007/978-90-481-2810-5_20 (ver p. 1).

[FM08] Luiz Renato G. Fontes e Pierre Mathieu. " $K$-processes, scaling limit and aging for the trap model in the complete graph". Em: Ann. Probab. 36.4 (2008), pp. 1322-1358. ISSN: 0091-1798. DOI: 10.1214/07-AOP360. URL: http://dx.doi.org/10.1214/07-AOP360 (ver pp. 1, 42).

[FP13] Luiz Renato G. Fontes e Gabriel Ribeiro da Cruz Peixoto. "Elementary Results on K Processes with Weights". Em: Markov Processes and Related Filelds 19 (2 2013), pp. 343370 (ver pp. 1, 42).

[Gav11] Renato Jacob Gava. "Limites de escala do modelo de armadilhas numa árvore". PhD thesis. Instituto de Matemática e Estatística da Universidade de São Paulo, 2011 (ver pp. 1,3).

[JLT12] Milton Jara, Claudio Landim e Augusto Teixeira. "Universality of trap models in the ergodic time scale". Em: In preparation (2012). URL: arXiv:1208.5675 (ver p. 1).

[Kin93] J. F. C. Kingman. Poisson processes. Vol. 3. Oxford Studies in Probability. Oxford Science Publications. New York: The Clarendon Press Oxford University Press, 1993, pp. viii+104. ISBN: 0-19-853693-3 (ver pp. 5, 51). 
[Kol51] Andrei Nikolaevich Kolmogorov. "On the differentiability of the transition probabilities in stationary Markov processes with a denumberable number of states". Em: Moskov. Gos. Univ. Učenye Zapiski Matematika 148(4) (1951), pp. 53-59 (ver p. 1).

[Lau80] G. Laue. "Remarks on the relation between fractional moments and fractional derivatives of characteristic functions". Em: J. Appl. Probab. 17.2 (1980), pp. 456-466. ISSN: 00219002 (ver p. 30).

[Pei11] Gabriel Ribeiro da Cruz Peixoto. "Um estudo sobre o Processo K não homogêneo". Master's thesis. Instituto de Matemática e Estatística da Universidade de São Paulo, 2011 (ver p. 1).

[ST94] Gennady Samorodnitsky e Murad Taqqu. Stable Non-Gaussian Random Processes: Stochastic Models with Infinite Variance. $1^{\mathrm{a}}$ ed. Stochastic modeling. Chapman e Hall/CRC, 1994. ISBN: 0412051710 (ver p. 30).

[SN00] Munetaka Sasaki e Koji Nemoto. "Analysis on Aging in the Generalized Random Energy Model". Em: Journal of the Physical Society of Japan 69.9 (2000), pp. 3045-3050. DOI: 10.1143/JPSJ.69.3045. URL: http://jpsj.ipap.jp/link?JPSJ/69/3045/ (ver p. 1). 